\title{
Towards Full Aircraft Airframe Noise Prediction: Detached Eddy Simulations
}

\author{
Mehdi R. Khorrami* \\ NASA Langley Research Center, Hampton, Virginia, 23681 \\ and \\ Raymond E. Mineck ${ }^{\dagger}$ \\ Yorktown, Virginia 23692
}

\begin{abstract}
Results from a computational study on the aeroacoustic characteristics of an $18 \%$-scale, semi-span Gulfstream aircraft model are presented in this paper. NASA's FUN3D unstructured compressible Navier-Stokes solver was used to perform steady and unsteady simulations of the flow field associated with this high-fidelity aircraft model. Solutions were obtained for free-air at a Mach number of 0.2 with the flap deflected at $39^{\circ}$, with the main gear off and on (the two baseline configurations). Initially, the study focused on accurately predicting the prominent noise sources at both flap tips for the baseline configuration with deployed flap only. Building upon the experience gained from this initial effort, subsequent work involved the full landing configuration with both flap and main landing gear deployed. For the unsteady computations, we capitalized on the Detached Eddy Simulation capability of FUN3D to capture the complex time-dependent flow features associated with the flap and main gear. To resolve the noise sources over a broad frequency range, the tailored grid was very dense near the flap inboard and outboard tips and the region surrounding the gear. Extensive comparison of the computed steady and unsteady surface pressures with wind tunnel measurements showed good agreement for the global aerodynamic characteristics and the local flow field at the flap inboard tip. However, the computed pressure coefficients indicated that a zone of separated flow that forms in the vicinity of the outboard tip is larger in extent along the flap span and chord than measurements suggest. Computed farfield acoustic characteristics from a FW-H integral approach that used the simulated pressures on the model solid surface were in excellent agreement with corresponding measurements.
\end{abstract}

\section{Nomenclature}

$\begin{array}{ll}\text { AOA } & =\text { angle-of-attack } \\ \mathrm{C}_{\mathrm{L}} & =\text { lift coefficient } \\ \mathrm{Cp} & =\text { pressure coefficient } \\ \mathrm{C} & =\text { local chord } \\ \mathrm{DES} & =\text { detached eddy simulation } \\ \mathrm{DDES} & =\text { delayed detached eddy simulation } \\ \mathrm{FW}-\mathrm{H} & =\text { Ffowcs Williams-Hawkings } \\ \mathrm{GAC} & =\text { Gulfstream Aerospace Corporation } \\ \mathrm{Hz} & =\text { Hertz, cycles per second } \\ \mathrm{M} & =\text { Mach number } \\ \mathrm{MDDES} & =\text { modified delayed detached eddy simulation } \\ \mathrm{PSD} & =\text { power spectral density in psi }{ }^{2} / \mathrm{Hz} \\ \mathrm{psi} & =\text { pounds per square inch } \\ \mathrm{RANS} & =\text { Reynolds-Averaged Navier-Stokes } \\ \mathrm{Re} & =\text { Reynolds number } \\ \mathrm{RMS} & =\text { root mean square } \\ \mathrm{r} & =\text { radial distance } \\ \mathrm{S} & =\text { seconds } \\ \mathrm{X}, \mathrm{Y}, \mathrm{Z} & =\text { right handed coordinate system }\end{array}$

\footnotetext{
*Aerospace Engineer, Computational AeroSciences Branch, Associate Fellow AIAA

${ }^{\dagger}$ Consultant
} 


\section{Introduction}

Aircraft noise is an environmental hazard that affects metropolitan areas adjacent to major airports. With the projected growth in air travel, noise exposure levels are expected to increase substantially. NASA's Environmentally Responsible Aviation (ERA) project is involved with the development, evaluation, and maturation of novel noise reduction technologies that aim to confine aircraft noise footprints on the ground within airport boundaries. This is to be accomplished without detrimental effects to the aerodynamic efficiency of the aircraft. To most people, aircraft noise is synonymous with jet engine noise. During approach to landing, however, noise generated by the airframe is comparable to, and in some instances even louder than, propulsion noise. Acoustic measurements from flight tests ${ }^{1-6}$ and model-scale experiments ${ }^{7-8}$ have identified the landing gear and lift enhancing devices, such as wing slats and flaps, as the dominant airframe noise sources. Efficient design and development of viable noise reduction technologies demand the availability of high-fidelity simulation tools with accurate predictive capabilities. Ultimately, the goal of creating such tools is to promote a paradigm shift in the design procedure from the current time-consuming and costly "cut-and-try" approach to a physics-based virtual design environment whereby the aeroacoustic evaluation of a noise reduction concept and its subsequent optimization can take place in an integrated fashion.

As part of the NASA-Gulfstream partnership on airframe noise research, a series of flight tests and model scale experiments is being performed with a Gulfstream aircraft as the baseline configuration. An 18\% scale, semi-span replica of a Gulfstream aircraft has been designed and fabricated specifically to conduct airframe noise studies and evaluate noise reduction concepts for mitigating landing gear, flap, and gear-flap interaction noise. Aeroacoustic testing of the $18 \%$ scale semi-span model was performed as a series of carefully planned entries in the NASA Langley Research Center (LaRC) 14- by 22-Foot Subsonic Tunnel (14x22). The initial entry, completed in November 2010, focused on acquiring global forces (lift and drag) and measurements of steady and unsteady surface pressures. Detailed accounts of that entry and the processed aerodynamic data are given in Khorrami et al. ${ }^{9}$ and Khorrami and Neuhart. ${ }^{10}$ The second 14x22 tunnel entry, completed in April 2013, was executed in two segments. The first segment was dedicated to simultaneous acoustic ${ }^{11}$ and surface pressure measurements, ${ }^{12}$ while the second segment was devoted to off-surface flow measurements ${ }^{13}$ for the nominal aircraft landing configuration.

Concurrent with the experimental test campaign, there is a comprehensive computational effort aimed at extending the state-of-the-art airframe noise prediction capabilities from component-level to full aircraft system-level configurations. A unique aspect of the NASA-Gulfstream collaboration is the fully complementary, and sometimes leading, role that the computational simulations play in paving the way for better design, evaluation, and testing of noise reduction technologies. The computational approach is built upon the knowledge and experience gained from previous aeroacoustic simulations of an isolated nose landing gear for a Gulfstream aircraft and other relevant configurations. ${ }^{14-19}$ Following those earlier nose landing gear simulations, the current full-aircraft airframe noise prediction effort is being pursued via execution of two distinct computational methodologies. In this paper, results obtained with NASA's FUN3D solver are presented. The code, a standard unstructured compressible Navier-Stokes solver, is used to perform detached eddy simulations (DES) of the $18 \%$ scale, semi-span Gulfstream aircraft model in landing configuration (with the main landing gear off and on). In a companion paper by Khorrami, Fares and Casalino, ${ }^{20}$ we utilize Exa Corporation’s PowerFLOW® Lattice-Boltzmann flow solver to conduct aeroacoustic simulations of the same model configurations.

\section{Simulated Model Geometry}

The simulated model geometry corresponds to an $18 \%$ scale, semi-span, high-fidelity reproduction of a Gulfstream aircraft (see Fig. 1). The model geometry consists of a fuselage, wing, flap, flow-through nacelle, pylon, and main landing gear. A full description of this model, including its instrumentation relative to the surface distribution of steady pressure ports and unsteady transducers on various components, is provided in Refs. 9 and 10 . As an intermediate building-block configuration, the main landing gear was omitted in the initial comprehensive set of simulations used to determine solution convergence and other grid dependency issues. Figures 2 and 3, reproduced from Ref. 9, show the top view of the spanwise location and numbering of the static pressure rows on the model wing, winglet, and flap. Note in Fig. 2a that the wing pressure rows extend to include the area that overlaps the stowed flap top surface, shown in purple. This region represents the wing spoiler, which was treated as part of the wing for instrumentation purposes.

Figures 4 and 5, taken from Ref. 10, display the locations and numbering of the unsteady pressure sensors on the model flap used extensively to validate the present computations. Altogether, the flap has 40 dynamic sensors. These sensors (herein after referred to as probes) are distributed in three distinct areas: along the mid-span section (not shown) and two narrow chordwise strips adjacent to the inboard (see Fig. 4) and outboard (see Fig. 5) edges where significant flow unsteadiness is expected. Note that the outboard flap side-edge has a cavity that nearly spans the 
entire chord length. In the actual aircraft, this cavity houses a bulb seal that prevents metal to metal contact during flap retraction. To document whether the cavity acoustic modes get excited at high flap deflections, two probes (P26 and P27) are installed inside the cavity. The simulated geometry also includes this cavity and the bulb seal within it. Figures 6 through 8, also taken from Ref. 10, display the positions and numbering of the unsteady probes on the main landing gear and gear cavity door. In addition, both (outer and inner) wheels have a circumferential row of steady pressure ports.

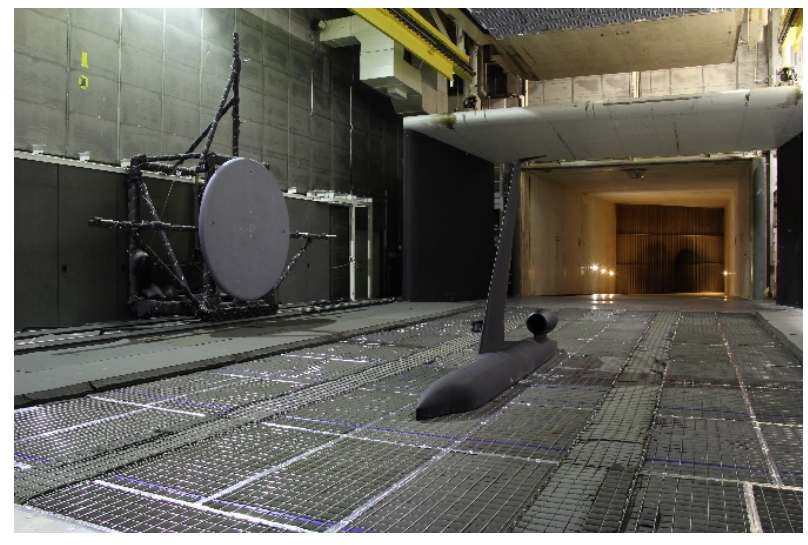

a) Installed in NASA LaRC 14x22 tunnel

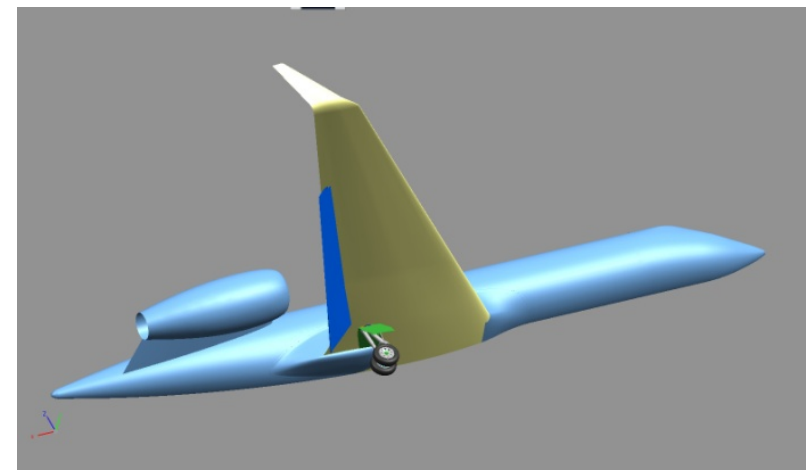

b) CAD model

Figure 1. $18 \%$ scale semi-span model of a Gulfstream aircraft.

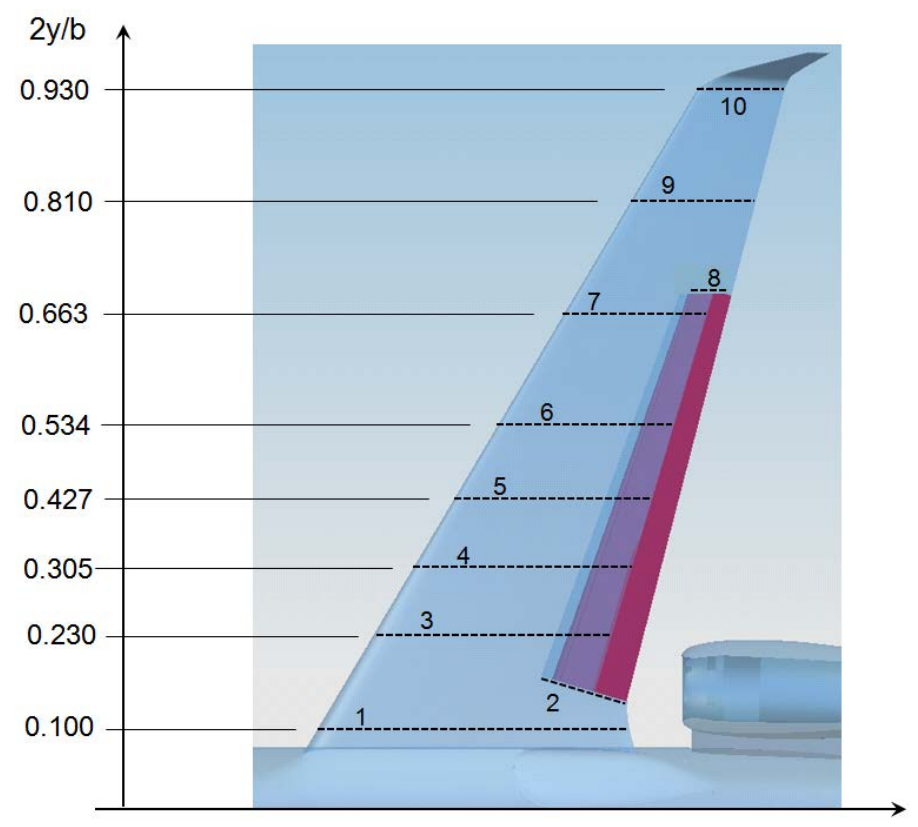

a) Wing

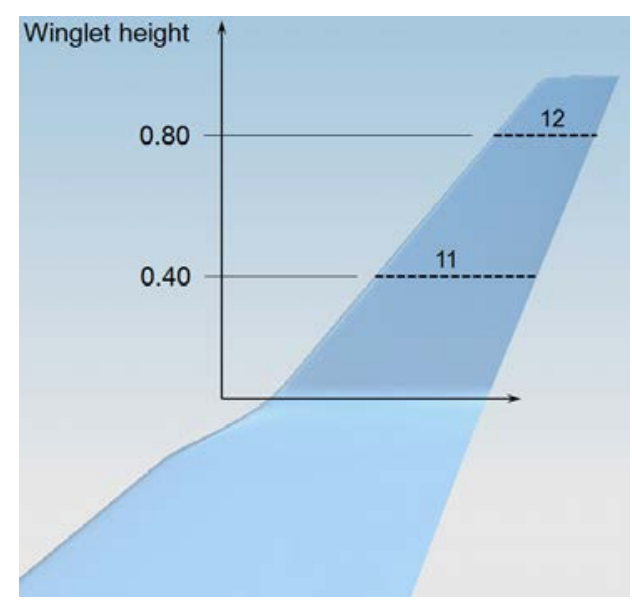

b) Winglet

Figure 2. Spanwise location and identification number for rows of static pressure orifices (from Ref. 9). 


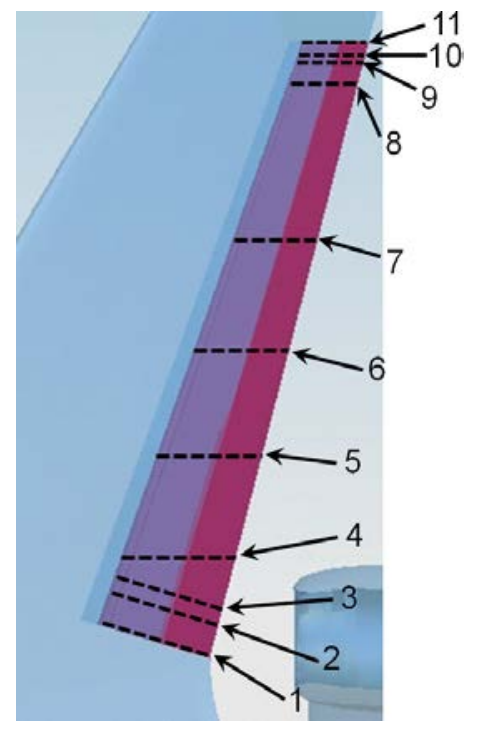

Figure 3. Flap orifice row distribution and numbering (from Ref. 9).

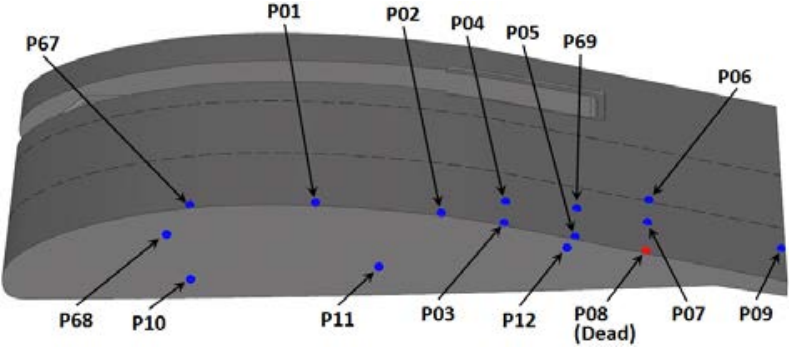

a) Top and side view

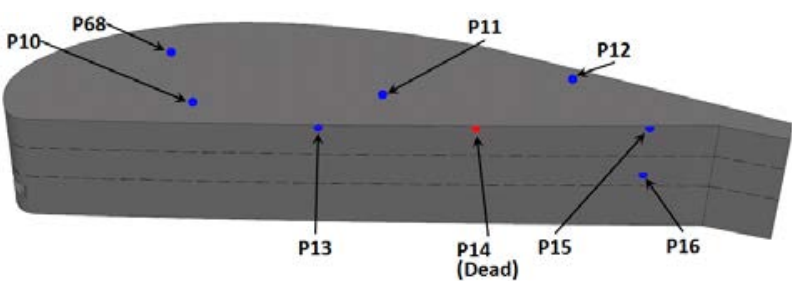

b) Bottom and side view

Figure 4. Locations and numbering of probes installed at the flap inboard edge (from Ref. 10).

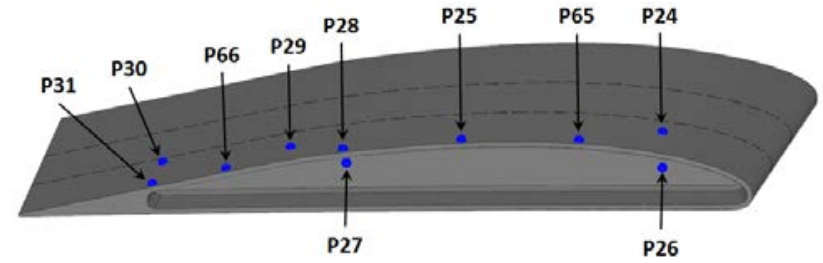

a) Top and side view

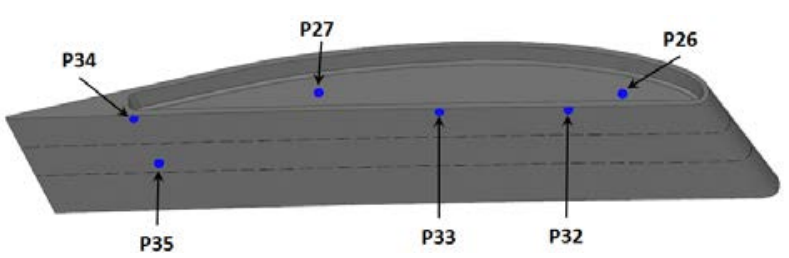

b) Bottom and side view

Figure 5. Locations and numbering of probes installed at the flap outboard edge (from Ref. 10). 


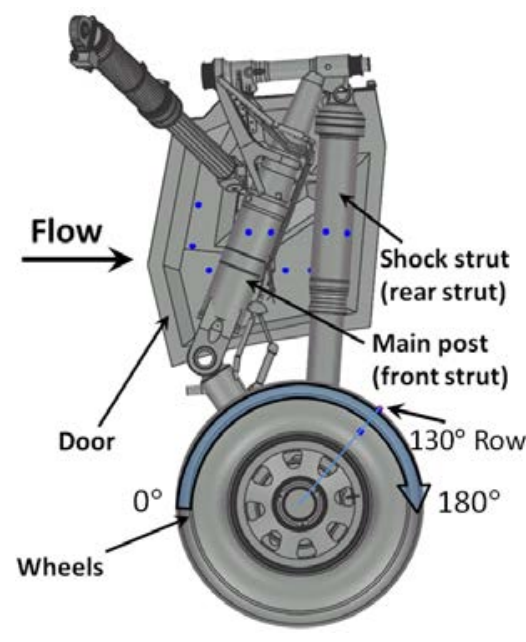

a) Side view

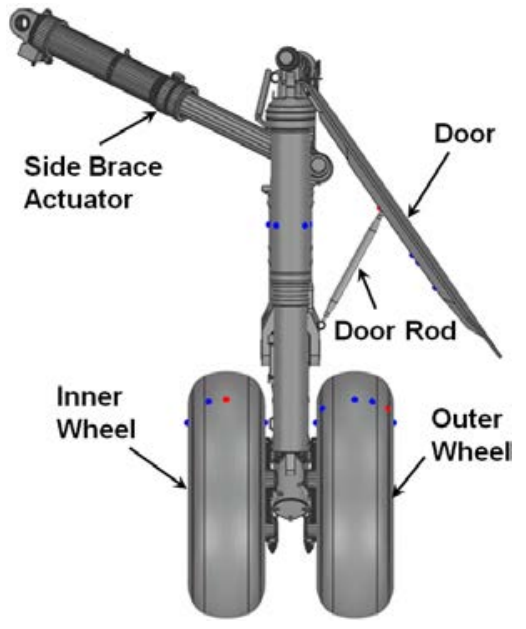

b) Rear view

Figure 6. Side and rear views of main landing gear highlighting major components (from Ref. 10).

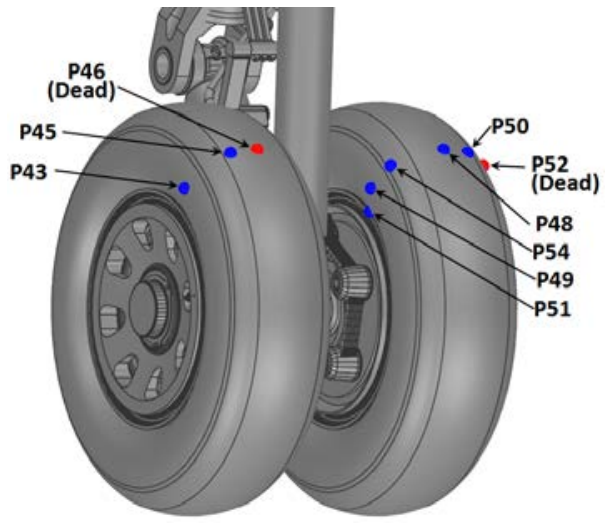

a) Interior (fuselage) view

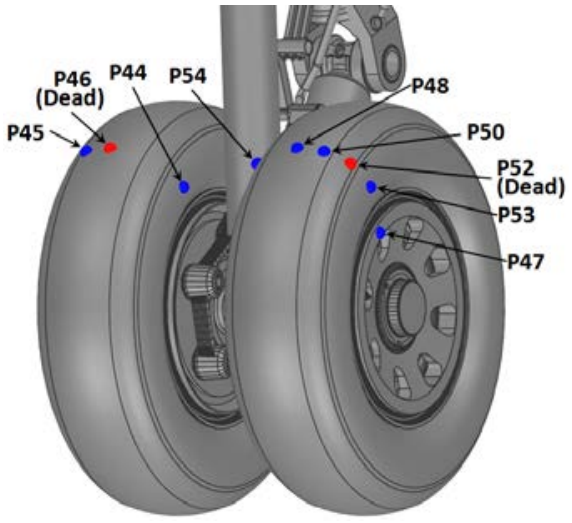

b) Exterior (wing-tip) view

Figure 7. Interior and exterior views of wheels showing locations of installed probes (from Ref. 10).

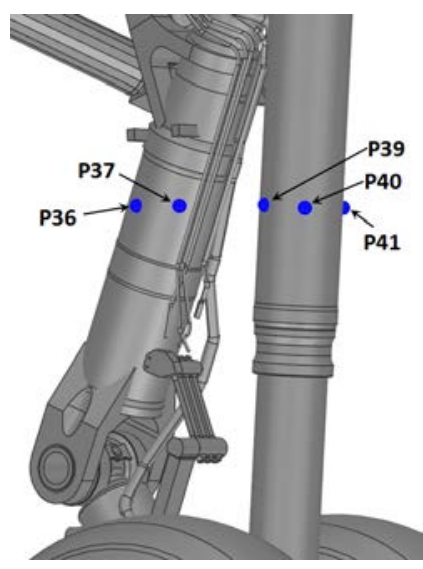

a) Interior (fuselage) view

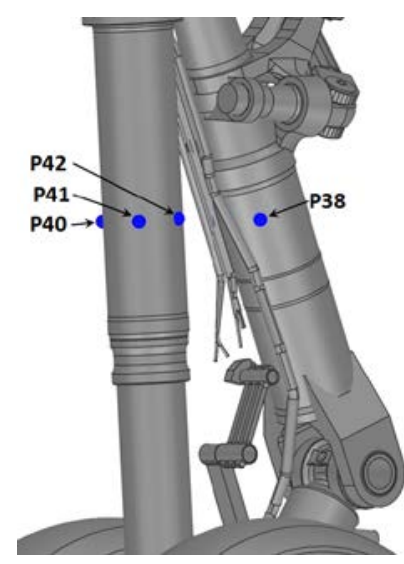

b) Exterior (wing-tip) view

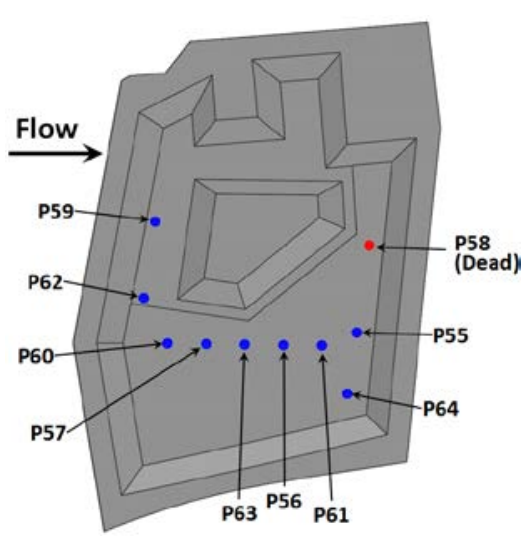

c) Door interior

Figure 8. Interior and exterior views of main gear post, shock strut, and door (from Ref. 10). 


\section{Computational Approach}

The numerical approach used in the present work is nearly identical to that outlined in Vatsa et al. ${ }^{15}$ For completeness, we present a brief summary of the key aspects of the computational procedure. For more details, the reader should consult Refs. 14 and 15.

\section{A. Flow Solver}

The NASA LaRC spatially second-order accurate CFD solver FUN3D was used to perform the simulations. FUN3D solves the discrete form of either the steady or time-dependent compressible Reynolds-Averaged NavierStokes (RANS) equations at the nodes of unstructured grids using a finite-volume discretization. The equations can be converged to a steady state or solved in time-accurate mode. Variable time steps at each node are used to accelerate convergence for steady flows. A constant time step at each node is used for unsteady flows (time- accurate mode). For steady flows, the solution at each time step is updated with a backward Euler time-differencing scheme and the use of local time stepping. At each time step, the linear system of equations is relaxed in a red-black fashion with a point-implicit algorithm. For time-accurate simulations, time is advanced using a second-order, backward difference approach. Several types of grid elements (tetrahedrons, pyramids, prisms, and hexahedrons) can be accommodated into the computational mesh. Various inviscid upwind flux functions, gradient limiters, and boundary conditions are available. The computational domain is decomposed into multiple parts with MPI communications to allow parallel computing on distributed memory systems.

\section{B. Turbulence Models}

FUN3D offers a wide selection of turbulence models. For steady calculations, the majority of the simulations were obtained using the one-equation Spalart-Allmaras (SA) model ${ }^{21}$ in conjunction with the solid-body rotation modification of Dacles-Mariani et al. ${ }^{22}$ To check the effects of the turbulence model on the steady solutions, the two-equation Menter shear-stress transport (SST) mode $^{23}$ was also used on several occasions. For unsteady simulations, following Vatsa et al., ${ }^{14,15}$ a Modified Delayed Detached Eddy Simulation (MDDES) methodology was utilized. The original DDES is a hybrid approach that uses the RANS equations with the Spalart-Allmaras model near the wall and a switching function to transition to the LES region in the rest of the computational domain. The DDES model applies a blending function to the destruction terms in the turbulence equation. In MDDES, the blending function is also applied to the production terms, but only outside of the near-wall region. As with any hybrid RANS/LES approach, the results obtained with this methodology are sensitive to characteristics of the flow solver, turbulence model, and overall grid topology used.

\section{Computational Grids}

The NASA TetrUSS suite ${ }^{24}$ was used to generate grids with only tetrahedral (tet) elements. The axis system was aligned with the vehicle body axis system (X along the line from the nose to the tail, Y from the centerline to the right wing tip, and $\mathrm{Z}$ pointing away from the ground, a right handed axis system). Locations and distances are presented in non-dimensional grid units using a length scale of one inch $(0.0254 \mathrm{~m})$. The nose of the fuselage was located at $(-0.72,-3.50,13.95)$, the end of the fuselage was located at $(184.68,-3.50,20.37)$, and the tip of the winglet was located at $(146.11,100.82,23.86)$. A series of developmental grids was generated to obtain values of $y+$ less than 1, and refined node spacing was used in regions with significant pressure gradients and in close proximity of the flap side edges. The farfield boundaries corresponded to the locations of the closed test section walls and the semi-span model mounted to the floor. The last grid in the series was tentatively chosen as baseline for this computational study. To assess grid convergence, the baseline was globally refined twice to create a family of three grids (baseline, refined 1, and refined 2) such that the surface node spacing of each successive grid was $92.5 \%$ of the parent coarser grid. Growth rate of the node spacing normal to the surface for the viscous nodes was similar for all three grids. Node spacing outside the viscous region was reduced in a similar manner. The three grids had $98.5 \times 10^{6}$, $124 \times 10^{6}$, and $151 \times 10^{6}$ nodes, respectively.

The baseline grid (98.5 $\times 10^{6}$ nodes) was modified to simulate the model in the open test section (free-air setup). The computational domain was a semi-cylinder with the inflow boundary at $X=-569.42$, the outflow boundary at $\mathrm{X}=1145.23$, and the farfield at a radius of 616.00 with the origin at $\mathrm{Y}=-3.75$. The viscous floor was embedded within the reflection plane. The viscous floor extended in the $\mathrm{X}$ direction from about -130 to about 329 and in the $\mathrm{Z}$ direction from about 132 to -105 . This grid was selected to represent a medium-resolution grid, herein referred to as grid 11. Nominal node spacing for the first node normal to the surface was $4.23 \times 10^{-5}$. The clustering of points normal to the surface was computed according to the VGRID stretching function ${ }^{25}$ 


$$
\delta \mathrm{n}=\delta 1\left[1+\mathrm{r} 1(1+\mathrm{r} 2)^{\mathrm{n}-1}\right]^{\mathrm{n}-1}
$$

where $\delta \mathrm{n}$ is the normal spacing of the $\mathrm{n}^{\text {th }}$ layer, $\delta 1$ is the spacing of the first layer, and the factors $\mathrm{r} 1$ and $\mathrm{r} 2$ are constants that determine the rate of stretching. (Note that if $\mathrm{r} 2$ is zero the stretching is geometric.) The two growth rate parameters to control the spacing of subsequent nodes normal to the surface were r1 $=0.160$ and r2 $=0.025$. Nominal node spacing at the farfield boundaries was 10.0. "Sources" used to control the node spacing in the grid generation process were defined to maintain a nominal node spacing near the flap inboard side edge of 0.025 and near the flap outboard side edge of 0.010 . Spacing in the region of the flap inboard tip where we anticipated the side-edge vortex to reside ranged from about 0.025 at the trailing edge to about 0.050 at one flap chord downstream. For the corresponding region near the flap outboard tip, the node spacing ranged from about 0.010 at the trailing edge to about 0.015 at one flap chord downstream. This grid had $2.0 \times 10^{6}$ surface nodes, $52.2 \times 10^{6}$ nodes in the viscous layers, and $102.6 \times 10^{6}$ total nodes. The initial node spacing away from the solid surface ranged from $0.9 \times 10^{-5}$ to $4.23 \times 10^{-5}$ with an average of $4.01 \times 10^{-5}$. The maximum number of viscous layers was 36 with about $95 \%$ of the surface covered by at least 23 layers. The value of $y^{+}$was less than 0.5 everywhere, with the exception of a few lines of nodes, one each along the wing and flap leading and trailing edges and upper edge of the vortex generators near the flap brackets. At these regions, the maximum value of $y+$ was closer to 1.2. Figure 9a presents a close-up view of the flap inboard edge geometry including the bracket and track nearest to the tip and the existing vortex generators. Figure $9 \mathrm{~b}$ displays the corresponding surface mesh showing the extremely fine resolutions achieved near the edge. A similar close-up view of the simulated geometry at the outboard tip is shown in Fig. 10a. In addition to the cavity, the tip geometry also includes a representation of the bulb seal that resides near the edge on the interior bottom surface of the cavity. The corresponding surface grid at the outboard tip is displayed in Fig. 10b. As can be seen in this figure, the constructed mesh is rather fine thus providing a reasonable resolution of the expected complex unsteady flow that is established at this tip. The viscous region adjacent to the solid surfaces is composed of semi-structured tetrahedral elements. This semi-structured grid was subsequently converted to prismatic cells to create a mixed element grid. The resulting grid had total of $102.8 \times 10^{6}$ nodes, including $328.0 \times 10^{6}$ tetrahedrons, 92.0 x $10^{6}$ prisms, and $0.5 \times 10^{6}$ pyramids.

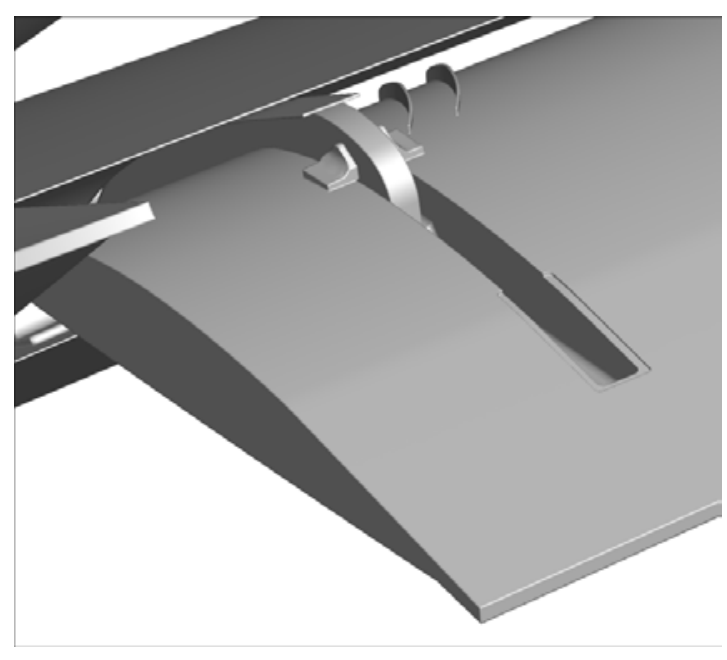

a) Model geometry

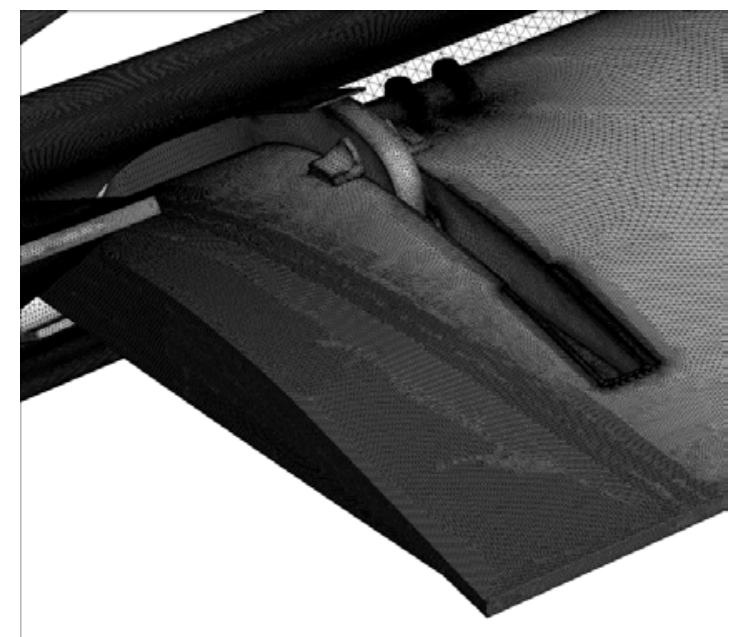

b) Surface mesh (grid 14)

Figure 9. Close-up view near flap inboard tip.

Grid 11 underwent a series of refinements to reduce node spacing in the region below the flap to allow extraction of the radiating acoustic waves directly from the FUN3D output for comparison with Ffowcs Williams-Hawkings (FW-H) analysis. Final node spacing in the refined region was 0.150. All other grid generation parameters were unchanged. This refined grid is herein referred to as grid 14. The additional "sources" used for this refinement had only a small influence outside of the immediate region. The number of surface nodes and viscous nodes increased slightly to $2.15 \times 10^{6}$ and $53.9 \times 10^{6}$, respectively. The total number of nodes increased dramatically to $146.3 \times 10^{6}$. This grid was also converted to contain mixed elements. That mixed element grid had $146.5 \times 10^{6}$ nodes from $557.2 \times 10^{6}$ tetrahedrons, $94.6 \times 10^{6}$ prisms, and $0.5 \times 10^{6}$ pyramids. Figure 11 displays streamwise planar cuts of grid 14 adjacent to the inboard and outboard flap tips, showing the enriched regions below, and downstream of, the side edges. 
The corresponding spanwise view is presented in Figure 12. The slightly slanted planar cut is aligned with the tapered and swept flap such that it bisects the flap chord at the inboard and outboard tips.

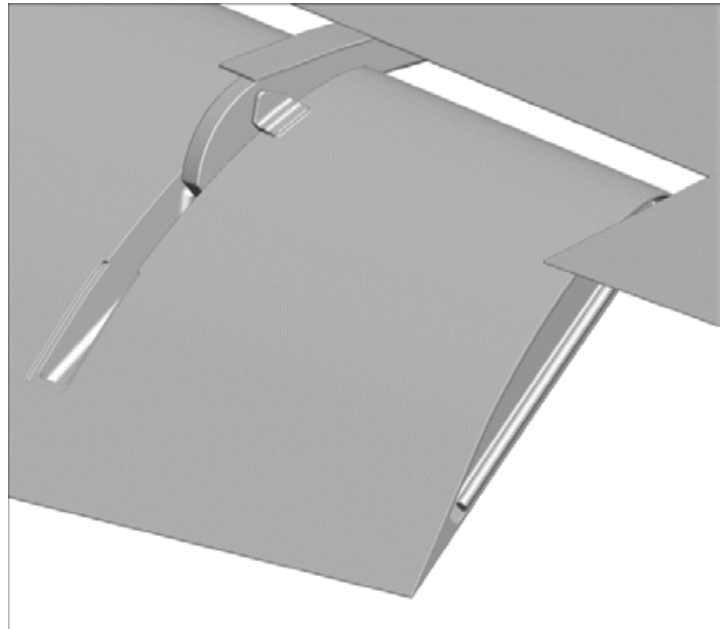

a) Model geometry

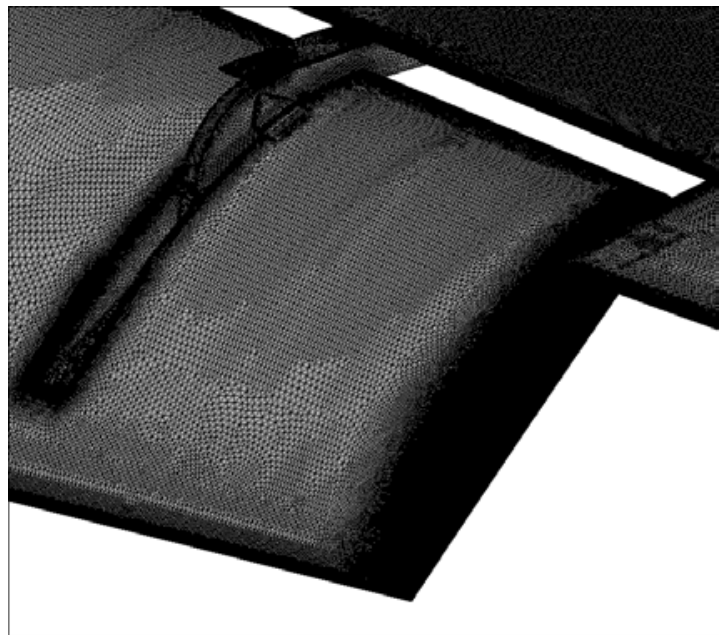

b) Surface mesh (grid 14)

Figure 10. Close-up view near flap outboard tip.

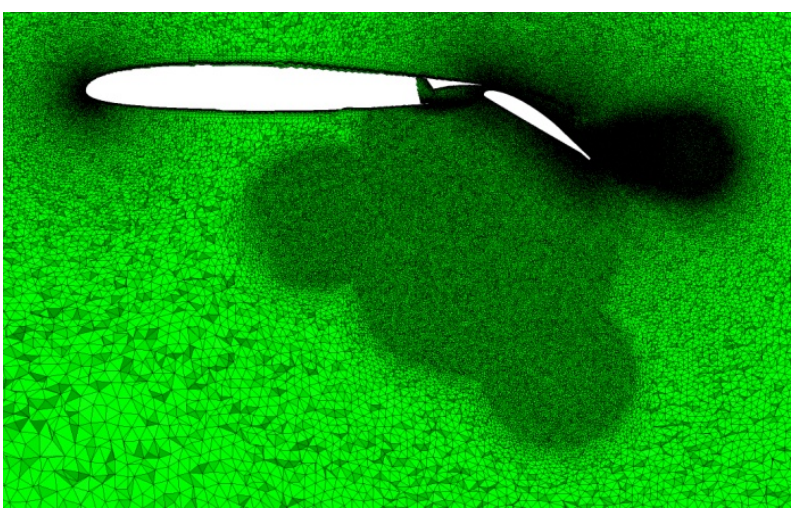

a) Inboard tip

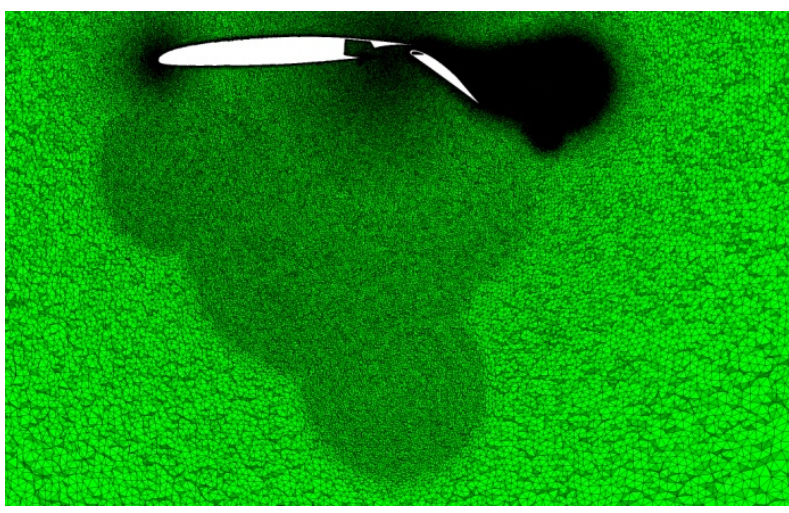

b) Outboard tip

Figure 11. Streamwise planar cut of grid 14 showing regions of enriched resolution adjacent to flap tips.

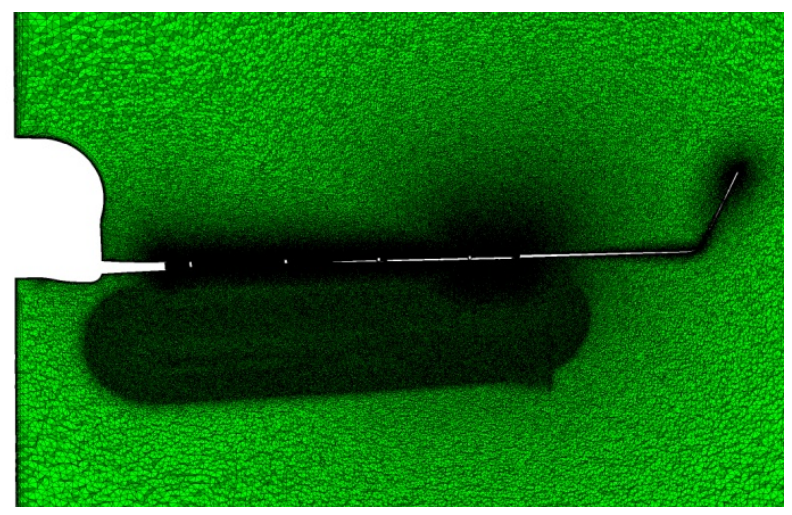

Figure 12. Spanwise planar cut of grid 14 showing extent of high resolution regions surrounding flap. 
After the first simulation using grid 14, the grid went through one last set of refinements. For this round, the node spacing in the volume surrounding the flap side edges was further reduced by $40 \%$. All other grid generation parameters used for the development of grid 14 were retained. The nominal node spacing inboard was 0.015 grid units and the nominal node spacing outboard was 0.006 grid units. This is referred to as the enriched grid 14 . It had $2.96 \times 10^{6}$ nodes on the surface and $232.9 \times 10^{6}$ nodes in the volume. Grid 14-enriched represents our finest resolution used to resolve the flow field at both flap tips.

For the baseline configuration with the main gear added, instead of the enriched version, the original grid 14 with a minor modification was used as the starting point in order to arrive at a manageable node count. That is, the parameters to reduce the node spacing below the wing-flap region for the FW-H comparison were eliminated since they were no longer needed. Relying on the experience gained from the nose landing gear simulations of Refs. 14 and 15, similar resolution guidance was maintained for the main landing gear in conjunction with grid 14 . The version of grid 14 with the main landing gear had $4.15 \times 10^{6}$ surface nodes. There were $243.9 \times 10^{6}$ nodes in the volume mesh. Given the relatively large size of this grid, no further refinements in regions surrounding the main gear were attempted. Figure 13 shows a close-up view of the main gear surface mesh and a streamwise planar cut of grid 14 bisecting the gear.

All vehicle surfaces and the viscous floor region were modeled with no slip boundary conditions. The remaining portion of the floor was modeled as a reflection plane. The inflow boundary, the outflow boundary, and the farfield boundary conditions were based on the Riemann invariants.

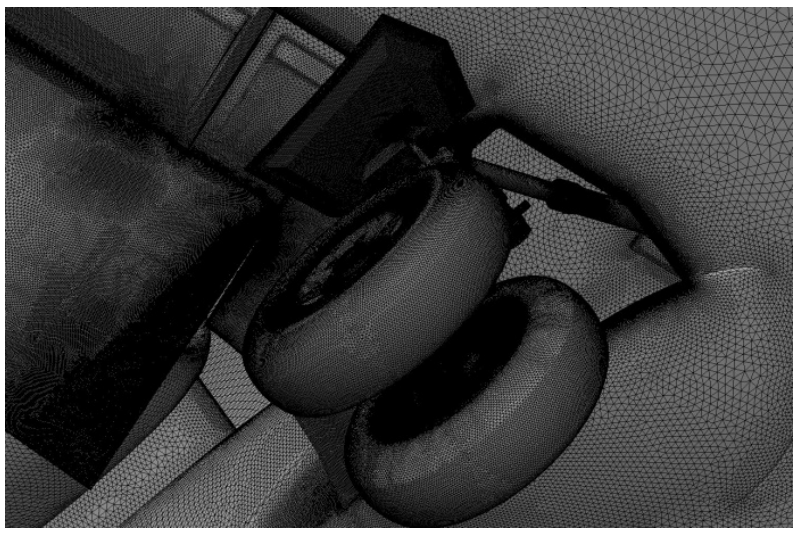

a) Surface mesh

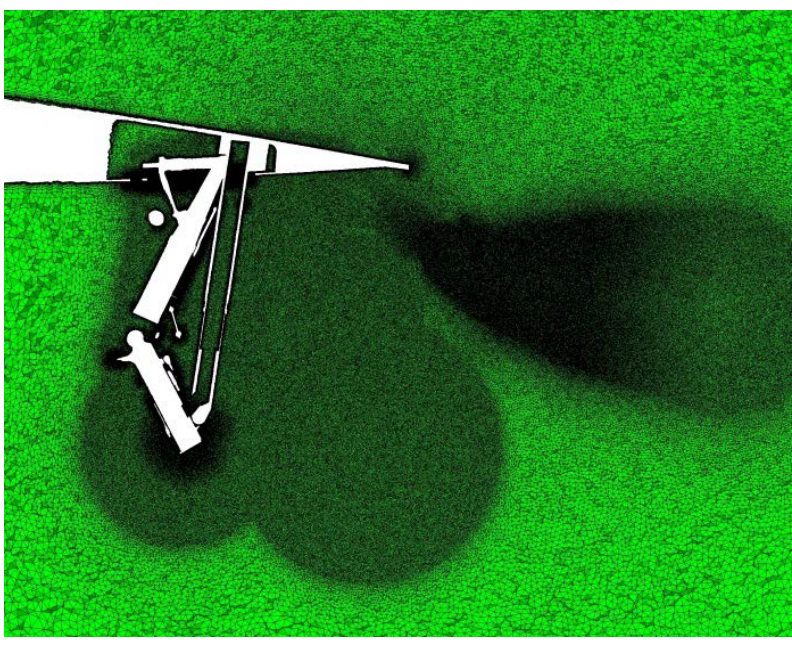

b) Planar cut bisecting gear

Figure 13. Close-up view of main gear of grid 14.

\section{Computational Procedure}

FUN3D was initially run in steady mode starting from free stream conditions with 500 first order iterations to quickly establish the general flow field. The CFL number was ramped linearly over 200 iterations from 1 to 25 for the flow solver and from 1 to 5 for the turbulence equation. When convergence problems were encountered, the CFL number was adjusted to improve convergence whenever possible. The inviscid flux construction used Roe's scheme with no limiter. The SA turbulence mode ${ }^{21}$ with Dacles-Mariani modification ${ }^{22}$ was used for the steady calculations. The steady equations were typically run for about 5,000 iterations, at which point the integrated force and moment coefficients were starting to settle within a reasonable band. FUN3D was then run in time accurate mode, with 14 iterations per time step using the MDDES approach. A time step $(\Delta \mathrm{t})$ of $2.5 \times 10^{-6} \mathrm{~s}$ was used for the current simulations.

\section{Results and Discussion}

During the 2010 14x22 wind tunnel entry, ${ }^{9}$ the aerodynamic measurements of the $18 \%$ scale, semi-span model were obtained with the tunnel in both closed (hard) wall and open-wall (open-jet) configurations. The wall corrected model aerodynamic forces (e.g., lift coefficient) representing free-air conditions were in good agreement with the open-jet data, with the latter configuration producing slightly less lift at all AOA (see Fig.14). It was also found dur- 
ing that experiment that, for the nominal landing configuration, the fluctuating surface pressures on the model flap and main landing gear were virtually identical for both tunnel configurations. In addition, the acoustic and off-surface (particle image velocimetry) flow measurements of 2013 required that the tunnel be in an open-wall (open-jet) configuration. Therefore, a decision was made to conduct the majority of the computations with the model in a freeair setting. The extensive set of initial simulations was performed for AOA of $3^{\circ}$, flap deflection angle of $39^{\circ}$ (landing configuration), and with the main landing gear removed. As mentioned earlier, this configuration provided an excellent intermediate geometry for benchmarking our computational approach before the more resource intensive configuration involving the main landing gear was attempted.

The computations in FUN3D were performed in a non-dimensional fashion. The scales used in our normalizations are the freestream speed of sound, density, kinematic viscosity, and a unit length of 1.0

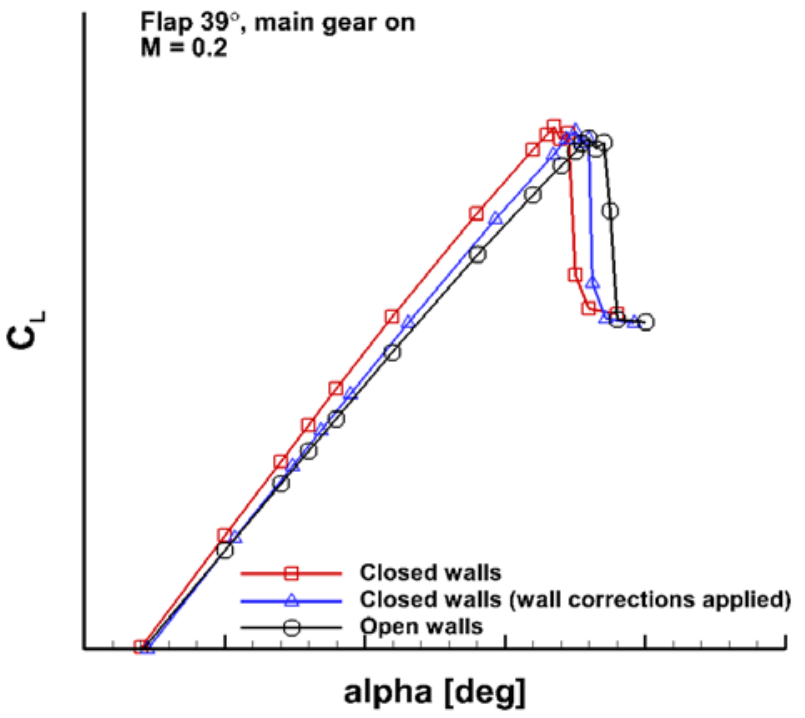

Figure 14. Tunnel configuration effects on lift coefficient. inch $(0.0254 \mathrm{~m})$. For the present case, reference flow variables were set to match conditions at the $14 \times 22$ wind tunnel test section entrance. All simulations were obtained for a freestream Mach number of 0.2. The corresponding unit Reynolds number is $1.33 \times 10^{6}$ per foot ( $4.40 \times 10^{6}$ per meter), resulting in a Reynolds number of $3.40 \times 10^{6}$ based on the $18 \%$ scale model mean aerodynamic chord of $30.8 \mathrm{in}$. $(0.782 \mathrm{~m})$. Because both upper and lower surfaces of the wing were tripped in the experiment, ${ }^{9}$ the computations were conducted in a fully turbulent mode.

For the purpose of determining the grid convergence behavior of the solution, the initial set of computations involved running FUN3D in steady RANS mode with the model in the walls-closed wind tunnel configuration. Since the entire test section was simulated, this configuration afforded us a more direct comparison with the experimental measurements without having to apply any tunnel wall corrections. For this setup, the tunnel side walls and ceiling were treated as slip (inviscid) boundaries. The three-grid family described in section III.C (baseline, refined 1, and refined 2) was used for this purpose. The computed solutions pointed to the presence of flow unsteadiness that is mainly associated with the flap. The unsteadiness was manifested as low amplitude pseudo oscillations in the lift and drag coefficients about their respective mean values. The difference in the lift coefficient between the baseline grid and the refined 1 grid was $0.1 \%$ and between the refined 1 grid and the refined 2 grid was $1.4 \%$. Similarly, the difference in the drag coefficient between the baseline grid and the refined 1 grid was $0.2 \%$ and between the refined 1 grid and the refined 2 grid was 1.3\%. The values predicted by FUN3D for the refined 2 grid and those measured in the wind tunnel differ by about $3.5 \%$ for the lift coefficient and by approximately $0.5 \%$ for the drag coefficient. Since the change in simulated lift and drag coefficients among the three grids is acceptably small, the baseline grid was chosen for the development of a grid to simulate the open jet test section. This grid is referred to as grid 11 .

These initial runs were mostly used to a) observe grid convergence behavior for the model integrated forces such as lift and drag coefficients, b) identify under-resolved flow regions and further refine the grid, c) decipher the effects of grid type on solution accuracy, d) determine turbulence model effects on flow solution, and e) achieve a modest level of grid independence for the computed solution. A full account of these initial runs is beyond the scope of the present paper. We will limit ourselves to describing the major findings that guided our choices of grid type and turbulence model.

The effect of turbulence model on the computed solution was studied by comparing the results obtained with the SA and SST models using grid 11 . The surface Cp distributions were found to be very similar with the computed lift and drag coefficients falling within $0.4 \%$ and $1.4 \%$ of each other, respectively. Thus, the more expensive SST turbulence model was abandoned and subsequent simulations used exclusively the SA model. Next, using both grids 11 and 14, unsteady simulations of the flow field were performed with an all-tetrahedral and a mixed-element mesh. Comparisons of the computed surface $\mathrm{Cp}$ and surface pressure fluctuations near the flap tips with measurements (not shown) clearly demonstrated that the mixed-element grid was producing more accurate results. This finding is in close agreement with the trends observed by Vatsa et al. ${ }^{14}$ for the case of a nose landing gear configuration. Thus, the all-tetrahedral version of the grid was abandoned in favor of the mixed-element grid and all subsequent simulations were performed using this mesh type. 


\section{A. Global View}

Before proceeding to quantitative comparisons between localized measurements and computations, a global picture of the flow field is presented. Figure 15 shows a representative plot of the computed steady surface pressure distribution over the semispan model with its flap deployed. The surface pressures display the footprint of strong suction peaks at the flap edges, especially the inboard tip; these peaks are attributed to the presence of strong streamwise vortices.

\section{Flap Inboard Tip}

A close-up view of the inboard edge, highlighted by the black rectangle in Fig. 15, with spanwise slices of the time-averaged static pressure is displayed in Fig. 16. As expected, most of the flow unsteadiness originates from the model flap. Although the simulated flap geometry is very complex,

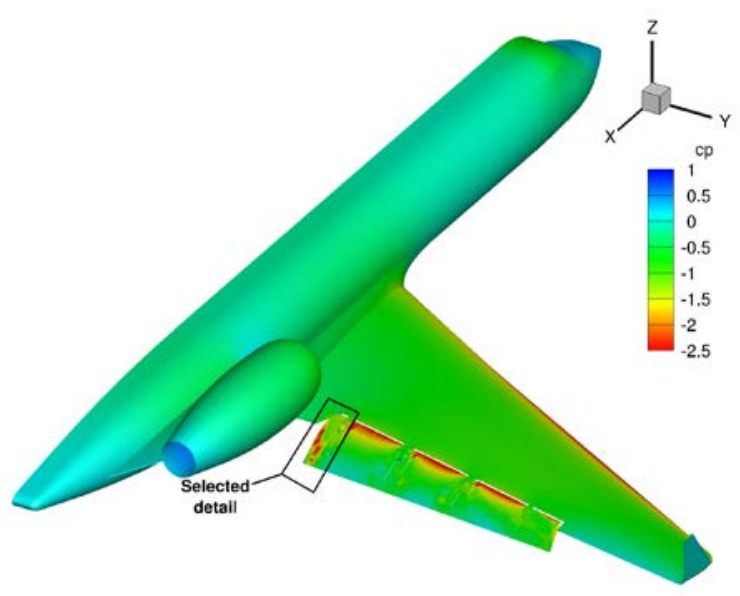

Figure 15. Computed steady surface pressure distribution on $18 \%$ scale semi-span model with flap deployed.

comprising several brackets and tracks, representative worm gears, and vortex generators, the major sources of flow unsteadiness reside mainly in the vicinity of the two flap side edges. Given the straight edge geometry of the inboard side-edge wall, the established flow field is typical of what has been reported in earlier studies. ${ }^{26,27}$ That is, existence of a strong pressure differential between the bottom and top surfaces of the flap results in the formation of a complex dual-vortex system. Near the flap leading edge, the boundary layer on the bottom surface separates at the sharp corner. The separated shear layers at the flap tips are rapidly deformed by Kelvin-Helmholtz instabilities, resulting in the formation of numerous vortex filaments of differing sizes and shapes. Roll-up of the deformed shear layers produces two prominent streamwise vortices situated along the lower and upper edges of the flap tip. Both vortices gain strength and size along the flap chord because of continued roll-up of the vorticity sheets at the two sharp edges. Downstream of the flap mid-chord, the lower side vortex begins to interact and merge with the vortex on the top surface. Eventually, a single dominant streamwise vortex is formed. To illustrate the intricate vortex formation process, the $18 \%$ model instantaneous vorticity field based on the Q-criterion is shown in Figure 17. The generation of vortex filaments is clearly captured in the close-up view of the vorticity field at the inboard edge.

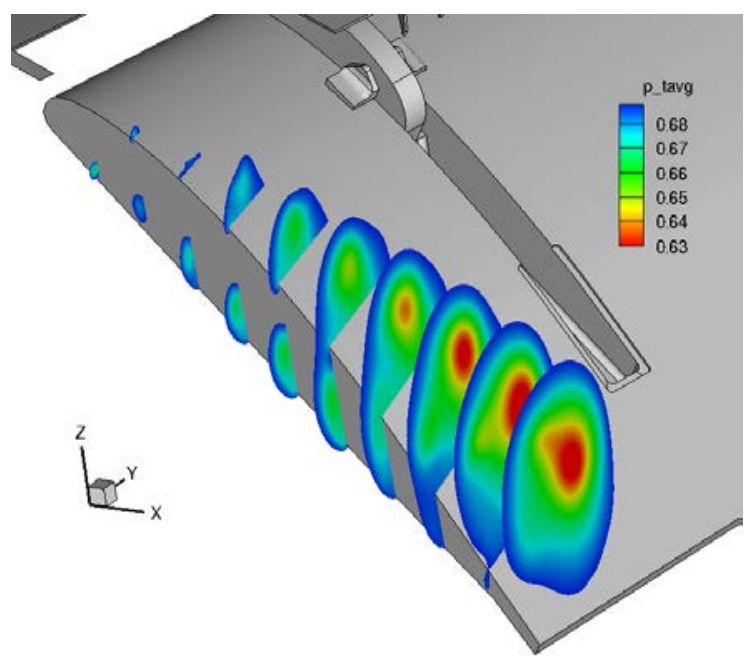

Figure 16. Time-averaged static pressure contours at the inboard flap side edge highlighting vortex cores. 


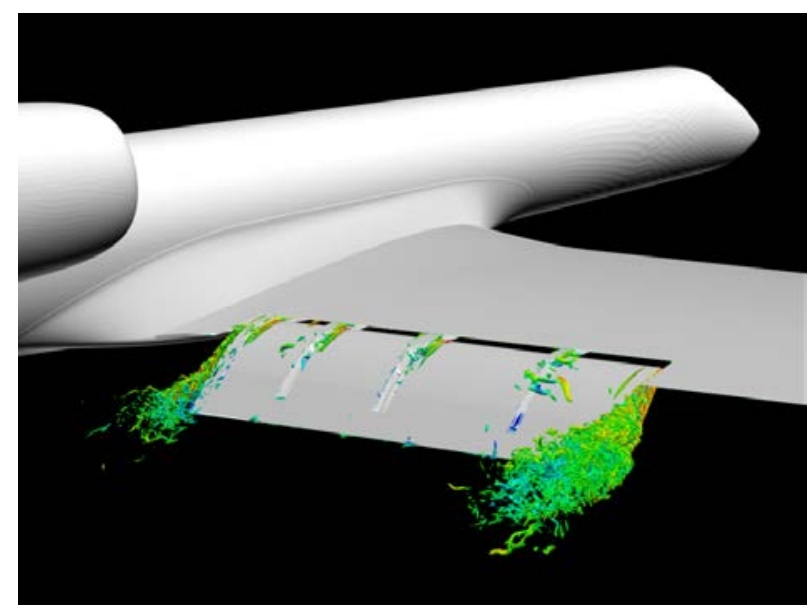

a) Global view of flap system

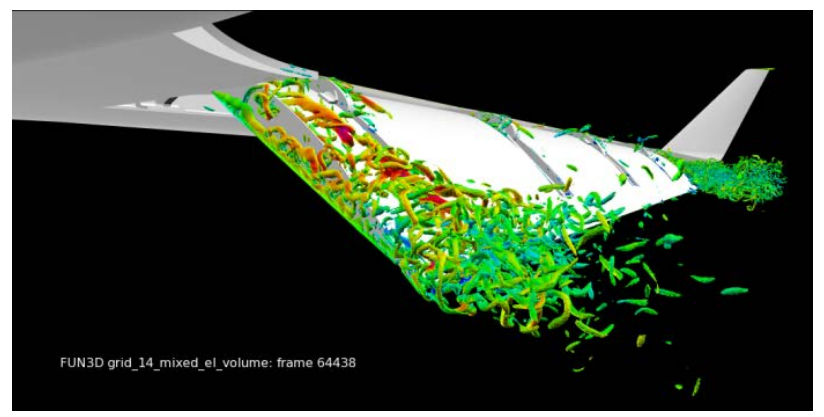

b) Close-up view of inboard edge

Figure 17. Instantaneous vorticity field based on Q-criterion.

\section{2. $\quad$ Flap Outboard Tip}

In contrast to the inboard tip, the outboard tip does not possess a straight-edge side wall that is typical of the simple model-scale geometries studied previously. Since the outboard tip houses a cavity and a bulb seal, its flow field should be quite complex and different than that described in the previous section for the standard straight-edge tip. However, the geometry of the outboard tip should not be viewed as being unique. Many of the civil transports in service today have flap tips with cavities, cut outs, bracket rollers, and other unusual depressions for flap deployment purposes.

Spanwise slices of the static pressure field at the outboard tip are shown in Fig. 18. Similar to the inboard tip, the pressure field depicts the formation of a dual vortex system. However, emergence of the vortical structures is not as well defined as for the inboard edge. Clearly, presence of the bulb seal and interaction of the side edge vortex with the tip cavity alter the dynamics of the flow field, shear layer roll up, and thus how the two vortices merge. Relative to the flow at the inboard edge, the primary vortex lacks a strong low pressure core. A snapshot of the instantaneous vorticity field is displayed in Fig. 19. The emergence of a vortex sheet initiated at the tip lower edge near the leading edge of the flap is clearly evident. Notice the rapid roll-up and disintegration of the vortex sheet into smaller vortex filaments caused by Kelvin-Helmholtz instabilities.

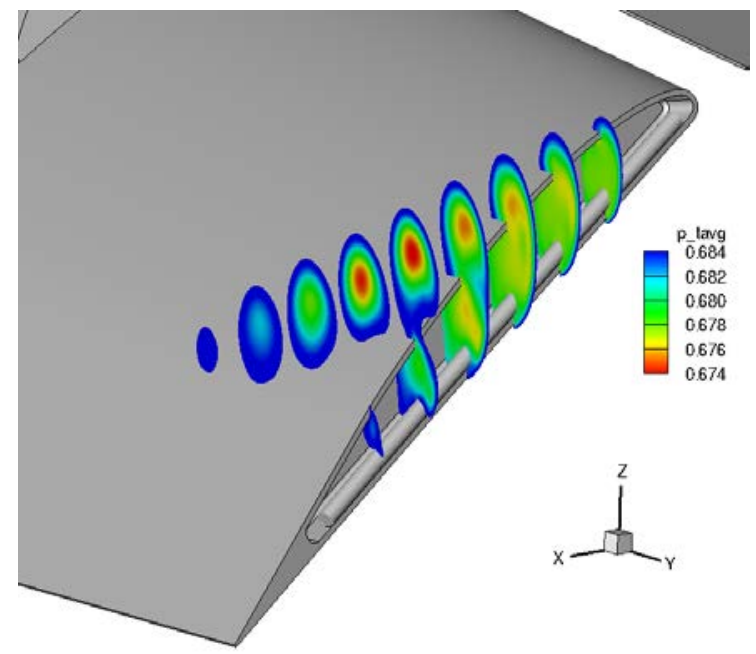

Figure 18. Time-averaged static pressure contours at outboard flap side edge highlighting flow-cavity interaction and formation of vortex cores.

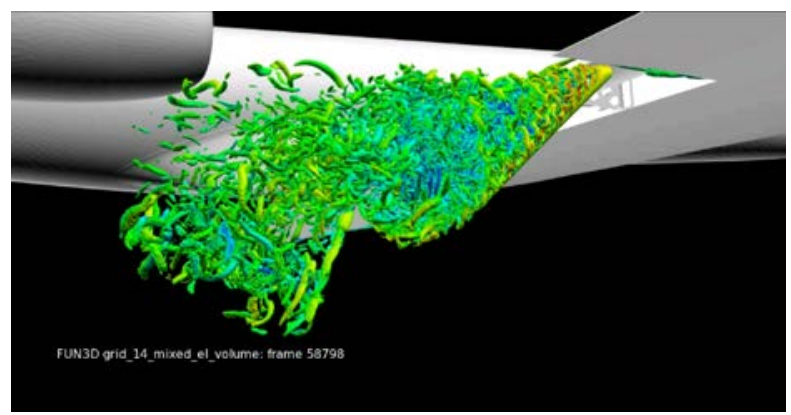

Figure 19. Close-up view of flap outboard edge showing instantaneous vorticity field based on Q-criterion. 


\section{Main Landing Gear}

Given the position of the main landing gear under the wing, gear deployment only affects the flow field at the flap inboard tip. An instantaneous snapshot of the vorticity field of the region containing the gear and inboard flap tip is presented in Fig. 20. The figure shows the presence of turbulent flow structures in the form of vortex filaments of various shapes, sizes, and strengths being shed from different gear subcomponents. Although the gear wake interacts with, and subsequently alters, the flow field at the inboard tip, a dual vortex system is maintained at the edge.

As was shown in the aerodynamic measurements of the $18 \%$ scale semi-span aircraft model, ${ }^{9-10}$ deployment of the main landing gear noticeably changes the steady and unsteady loading of the flap in the immediate vicinity of the inboard tip. This region includes the first two rows of the steady pressure ports (rows 1 and 2 in Fig.3). By the third flap row position, the differences in the surface pressure values for the configurations with and without the gear diminish to negligible levels.

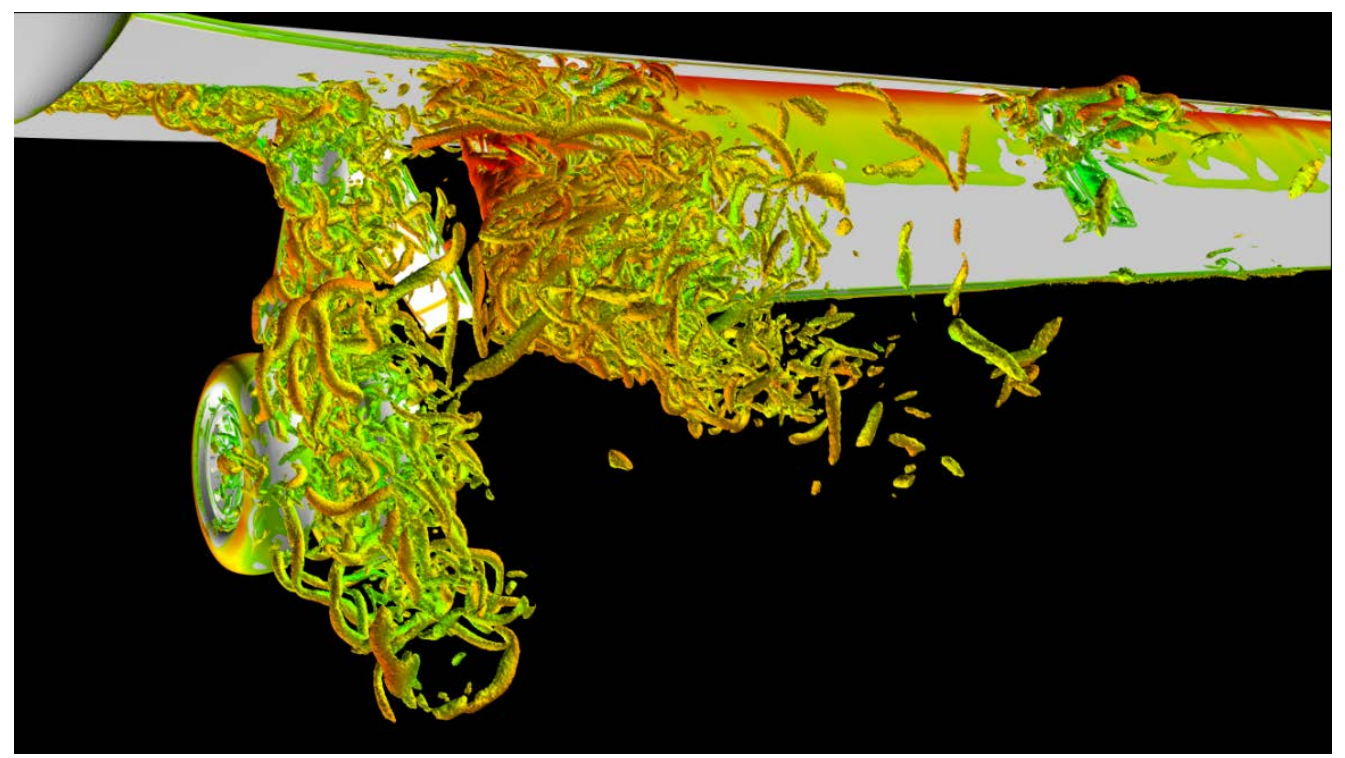

Figure 20. Close-up of view of instantaneous vorticity field based on Q-criterion showng gear-flap interaction.

\section{Acoustic Field}

Considerable flow unsteadiness (noise sources) is produced during the shear layer roll up, vortex formation, and vortex merging process, as well as by the interaction of the vortices with the sharp corners at the flap edge. A small portion of the energy associated with the pressure fluctuations (lift oscillations) is converted into sound waves that radiate outwardly away from the tips with a significant portion of the waves directed toward the ground. In addition, the presence of the cavity and the bulb seal at the outboard tip is expected to give rise to flow-cavity interaction and a possible cavity resonance within certain frequency bands. In general, because of the relative size of flow structures at the flap tips, it is anticipated that the lower frequency portion of the radiating spectrum will be dominated by the noise sources residing at the inboard tip and that the higher frequency range will be driven by the sources situated at the outboard tip.

A global view of the radiated sound field produced at the flap tips is given in Figure 21. The two-dimensional planar cut positioned at the flap mid chord clearly shows the origin of the sound waves, which propagate as concentric circles emanating from both tips (spherical radiation). The corresponding cut for the configuration with the main gear installed is presented in Fig. 22. Note from the figure that gear deployment does not diminish or significantly alter the sound levels nor the patterns associated with the inboard tip. However, as shown by the measurements presented in Ref. 11, deflection of the flap does reduce the noise levels produced by the main landing gear. 


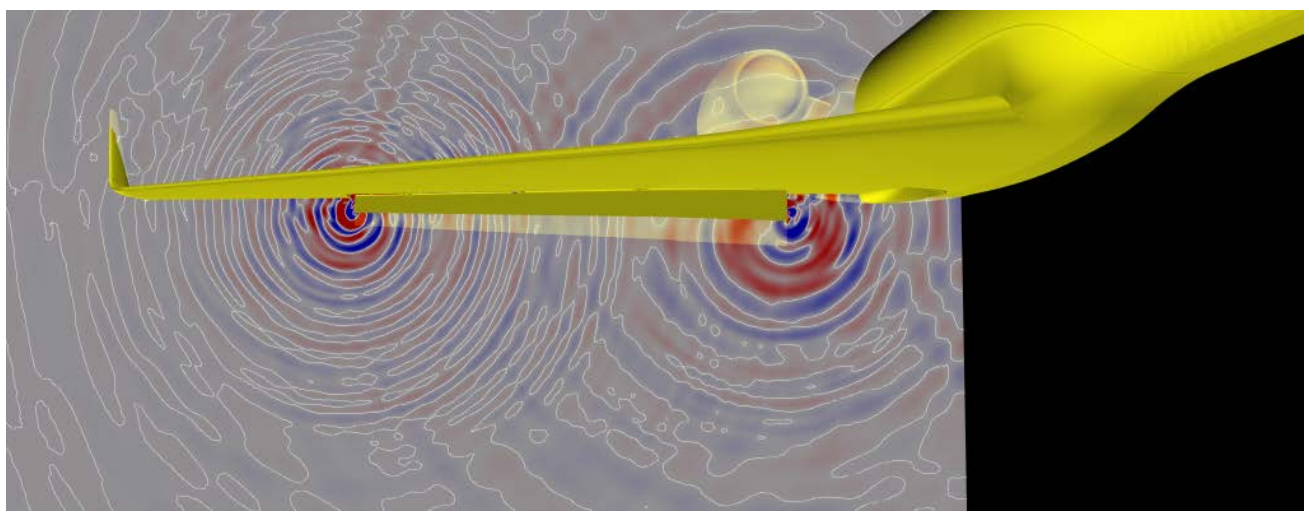

Figure 21. Radiated sound field associated with noise sources at inboard and outboard flap tips (planar cut at flap mid-chord).

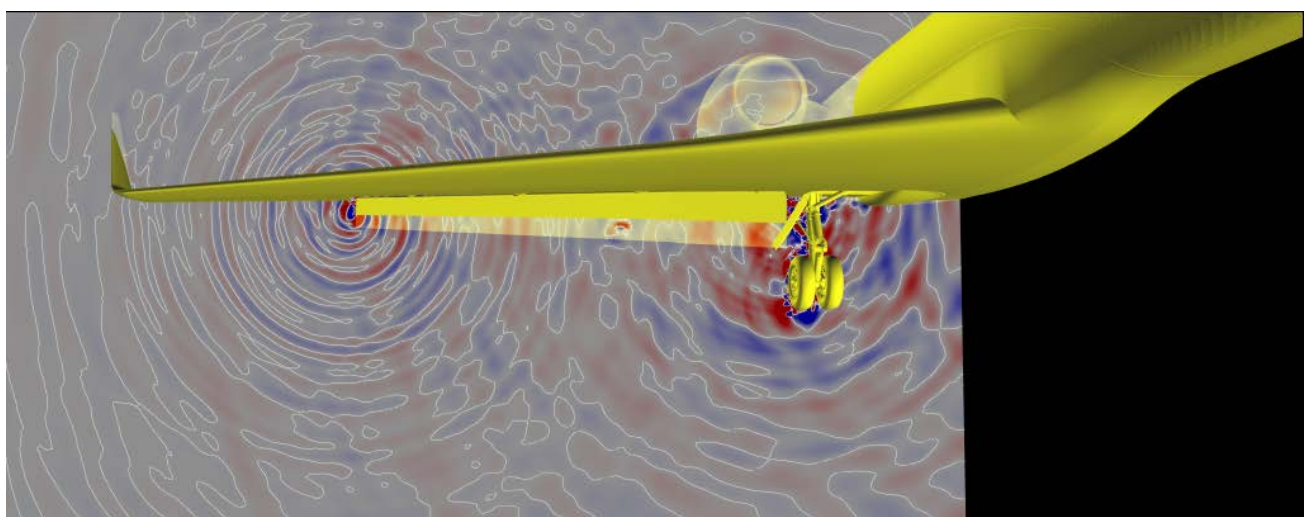

Figure 22. Radiated sound field for configuration with main landing gear deployed (planar cut at flap mid-chord).

\section{B. Surface Pressure Coefficient (Cp)}

The computed time-averaged $\mathrm{Cp}$ values for the wing were found to be in good agreement with the measured values at all the pressure port rows. A sample Cp plot comparing computed pressure coefficients from grids 11 and 14enriched with measurements for row 5 on the wing, corresponding to the flap mid-span location, is presented in Figure 23. As expected, nearly identical Cp values are produced by the two grids, indicating that the extra mesh refinements underneath the flap and close to the tips have had no noticeable effects on the magnitude of the lift being produced by the wing.

The Cp distribution for other rows on the wing and winglet are shown in Fig. 24. The computed Cp display higher suction peaks near the wing leading edge. The differences in suction peak amplitude between computed and measured values become more noticeable on the wing outboard segment and the winglet. In general, slightly better agreement between computed and measured $\mathrm{Cp}$ is obtained for the pressure rows closer to the wing root than for those situated in the wing mid-span and outboard sections. We expected better agreement between measured and computed $\mathrm{Cp}$ values from such highly refined grids than that observed in Fig. 24.

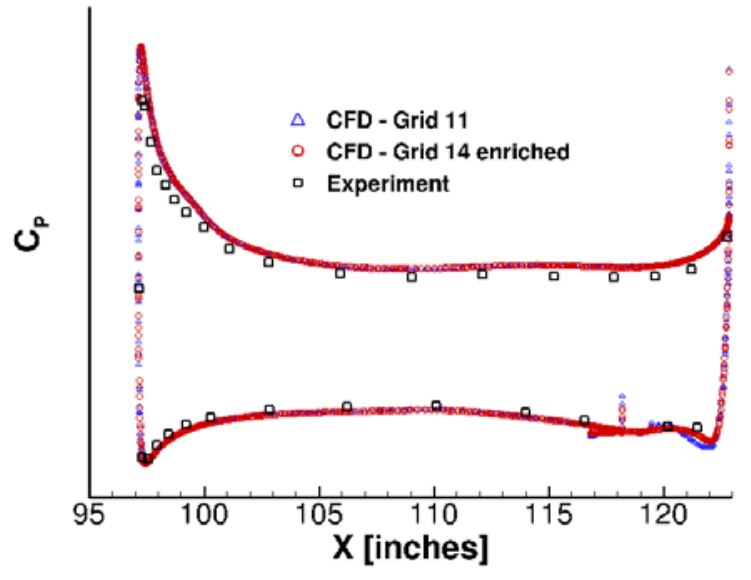

Figure 23. Surface pressures at wing row 5. 


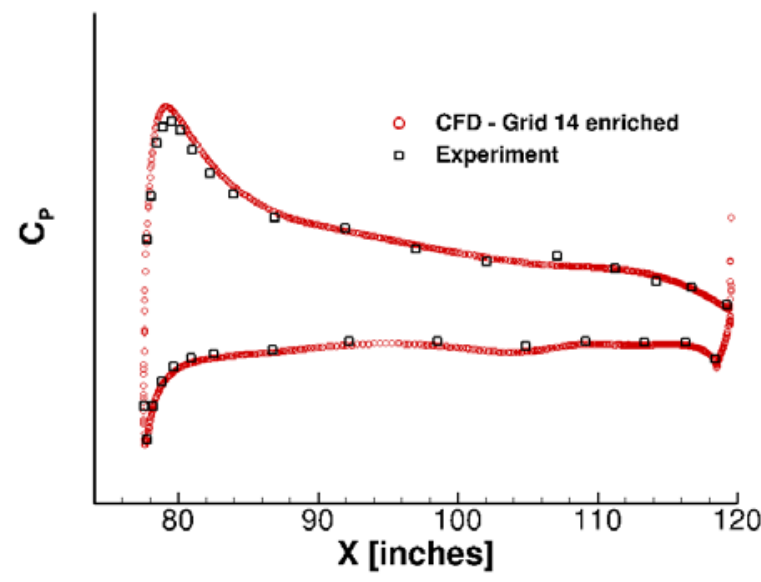

a) Wing row 1

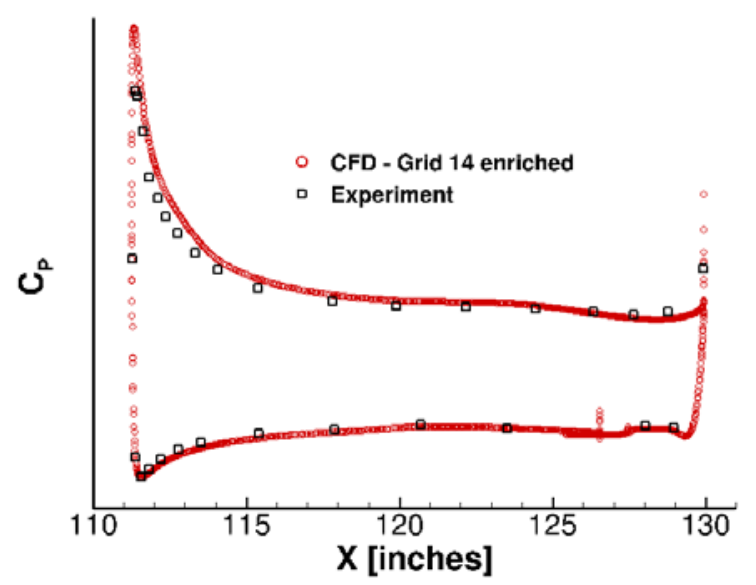

c) Wing row 7

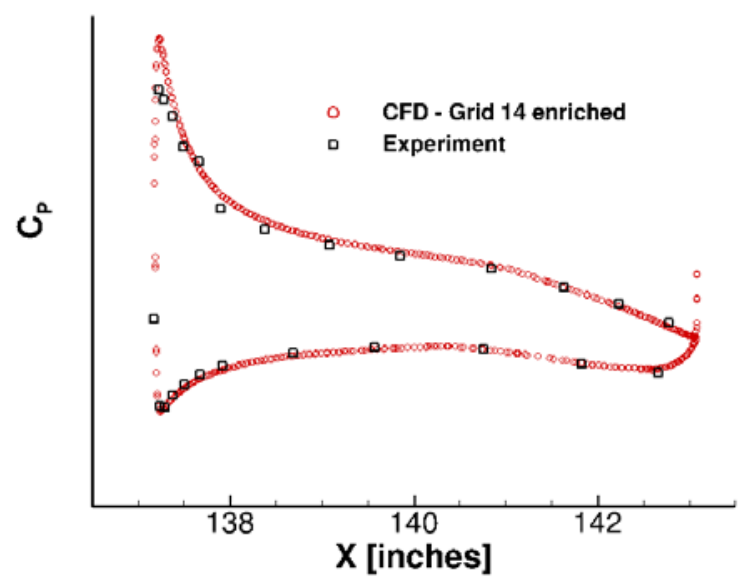

e) Winglet row 11

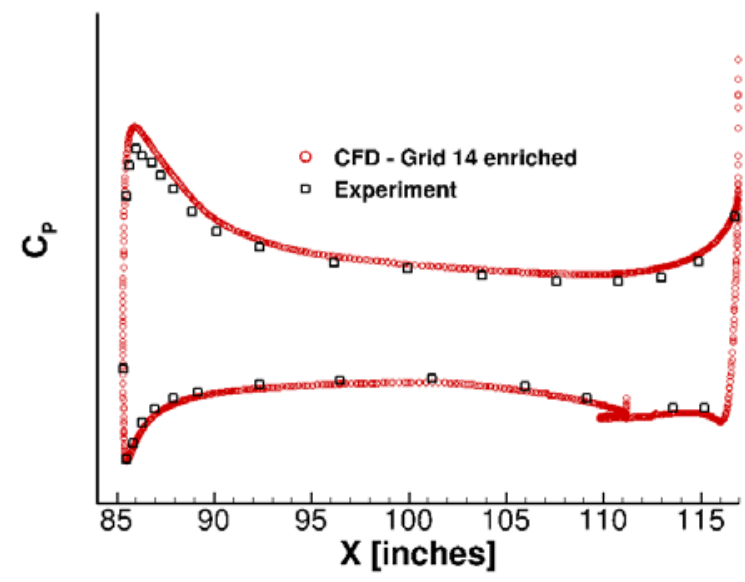

b) Wing row 3

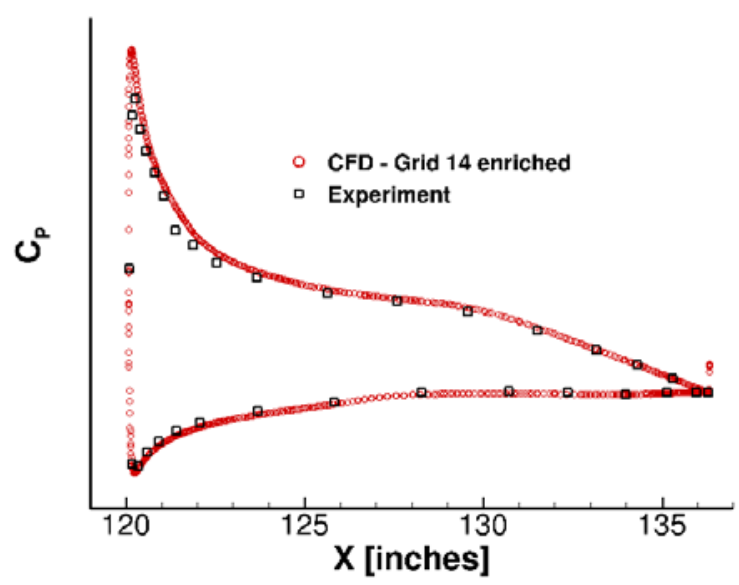

d) Wing row 9

Figure 24. Surface pressures on wing and winglet.

Similar trends in over-prediction of suction peak amplitude were also present in the companion computations of Ref. 20, where a Lattice Boltzmann approach was used to simulate the flow field around the same model. In fact, the present FUN3D wing Cp values are very close to those reported in Ref. 20. This suggests that the underlying causes 
for the observed discrepancies are not related to the flow solver nor to grid resolution issues, and reside elsewhere. There are three important sources that could produce such trends. The first is related to differences in model lift between open-jet tunnel and free-air settings. As can be seen in Fig. 14, the model produces slightly less lift in an open-jet setting than in free-air for the same AOA. In open-jet mode the effective angle of attack is slightly reduced due to jet turning, resulting in a lower suction peak along the wing leading edge. However, open-jet effects provide a partial answer only, and the small reduction in AOA is not sufficient to account for the differences in wing Cp values observed in Fig. 24. In our opinion, the more important factors are aeroelastic effects associated with the model and the effect of boundary layer development on the tunnel floor. With regard to the former, since the model has a high aspect ratio wing with a hollow structure, there is no doubt that it would have sustained deformation under load. This deformation would result in both untwisting of the wing and an increased dihedral angle (mostly in the outboard section). These two forms of aeroelastic deformation certainly can produce off-loading of the lift, particularly in the outboard section of the wing. In our simulations, the model geometry is treated as rigid and thus aeroelastic effects are neglected. With respect to the latter factor, as clearly shown in Neuhart et al., ${ }^{12}$ the presence of a thick boundary layer on the tunnel floor measurably lowers the model lift coefficient with the loss being manifested as reduced $\mathrm{Cp}$ values in the leading edge segment of the wing. Although in the present simulations the tunnel floor is treated as a viscous surface to partially account for this effect, consideration of its full impact by matching the boundary layer thickness at the computational inlet plane is beyond the scope of the current study.

The time-averaged Cp values at the flap mid-span location (row 6 in Fig. 3) are displayed in Fig. 25. The computed pressures from grids 11 and 14-enriched are virtually identical. Good agreement between computed and measured $\mathrm{Cp}$ is observed at this flap section. The pressure distributions at the two rows closest to the flap inboard edge are plotted in Figs. 26a and 26b (rows 1 and 2, respectively). The Cp values obtained from grid 11 provide good agreement with the measured pressures. Except for a small region on the bottom surface near the flap leading edge (Fig. 26a), the computed Cp values obtained with the higher resolution grid 14-enriched are in better agreement with the measurements. The cause of the discrepancy in Cp near the leading edge at this spanwise location is not yet clear to us. The Cp distributions at other flap rows are also shown in Fig. 26. Similar to the wing pressure distributions, the computations show slightly higher suction near the flap leading edge. The differences in leading edge pressures could partially be attributed to the aeroelastic effects of the tested model and/or deficiencies of the turbulence model.

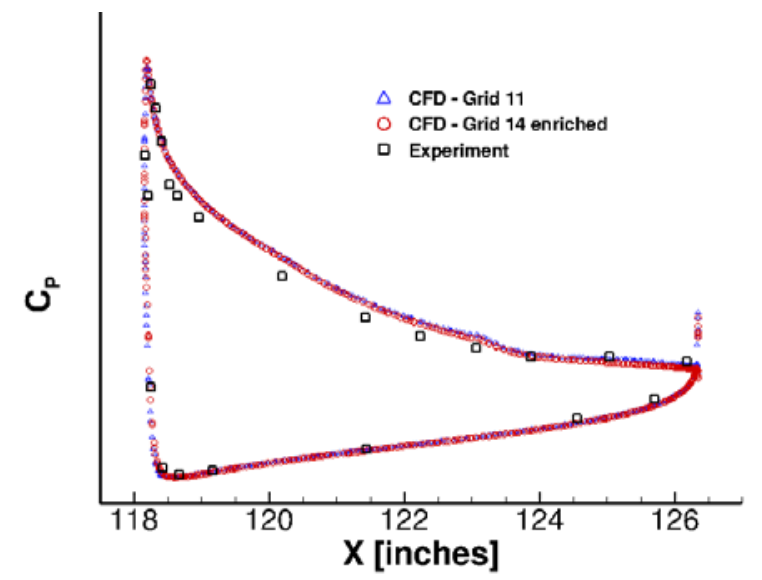

Figure 25. Surface pressures at the flap mid-span position (row 6).

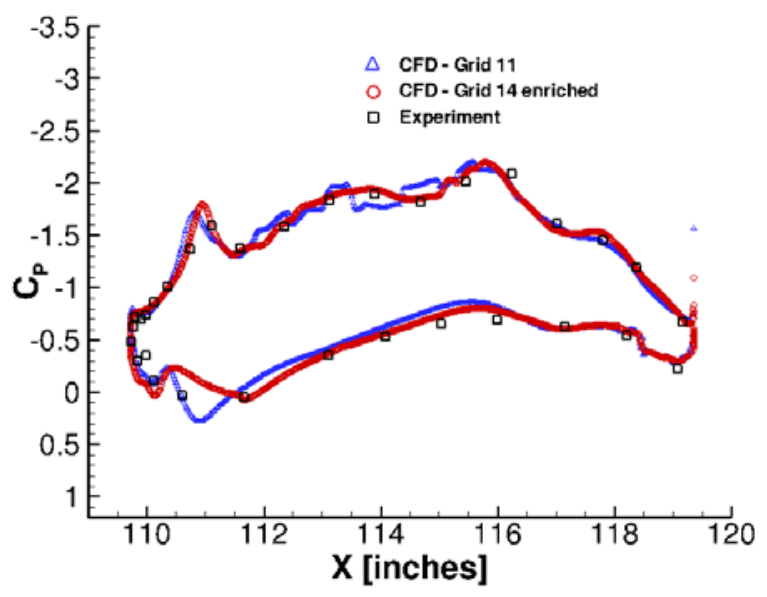

a) Flap row 1

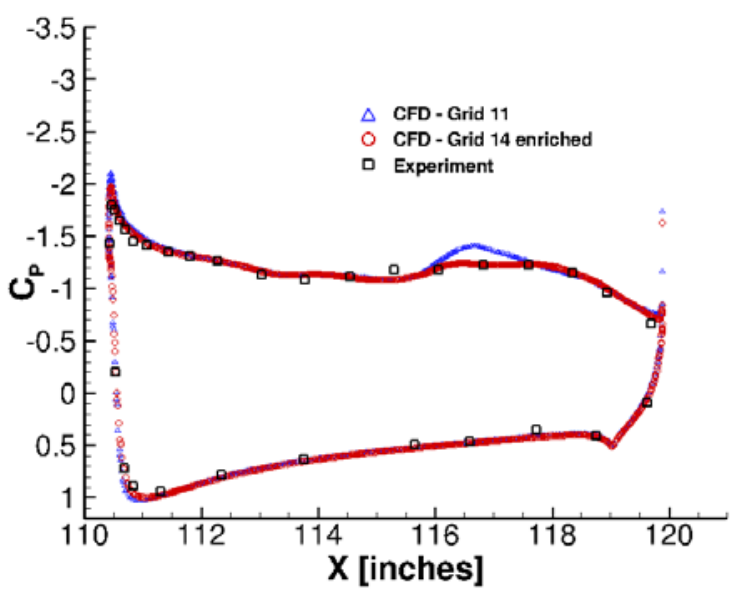

b) Flap row 2

Figure 26. Surface pressures on flap. 


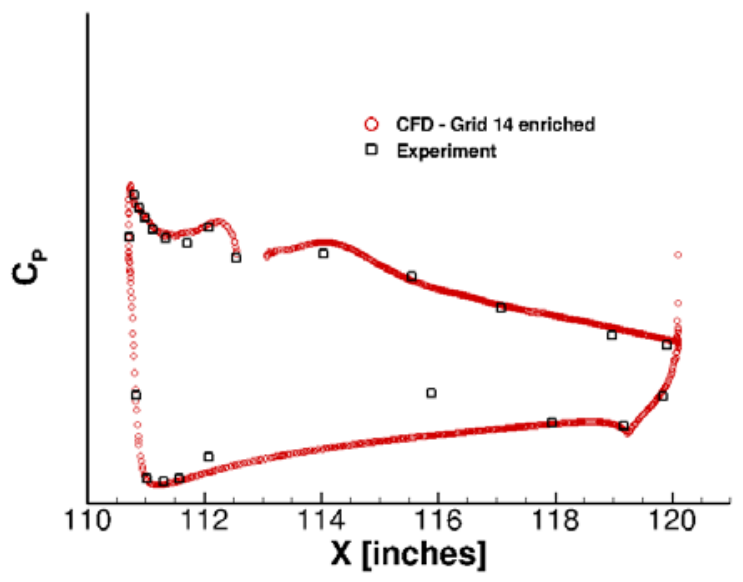

c) Flap row3

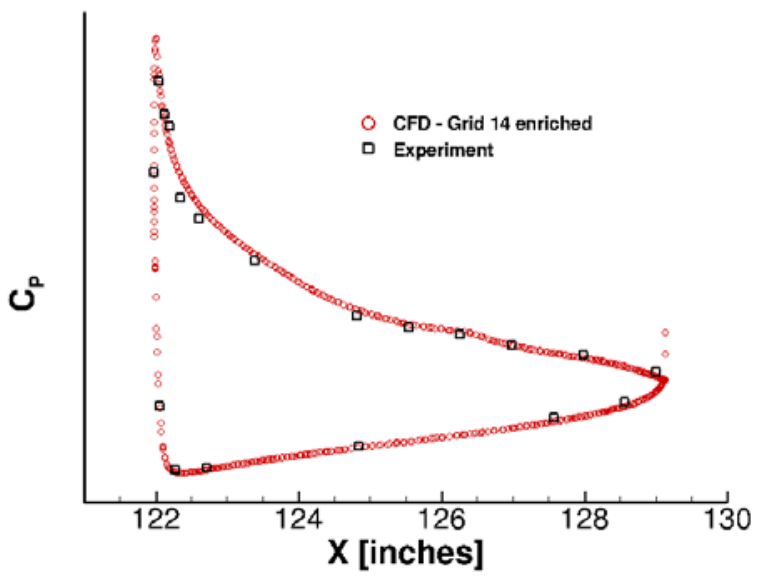

e) Flap row 7

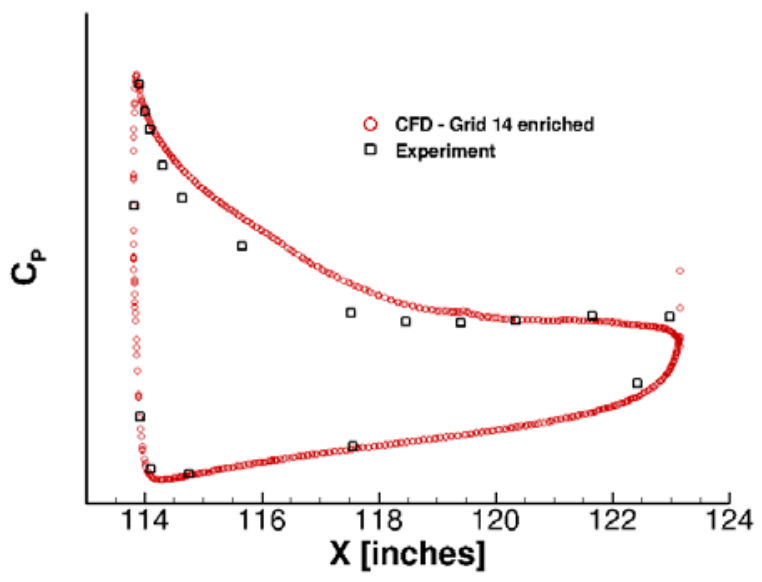

d) Flap row 5

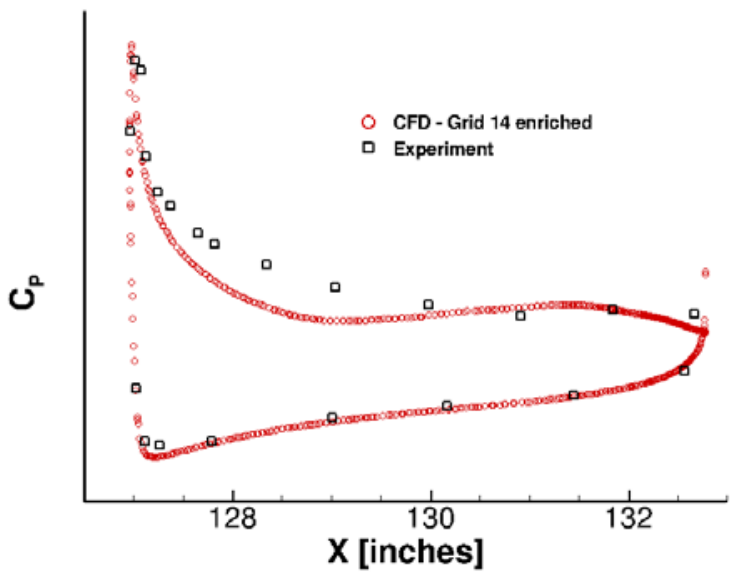

f) Flap row 9

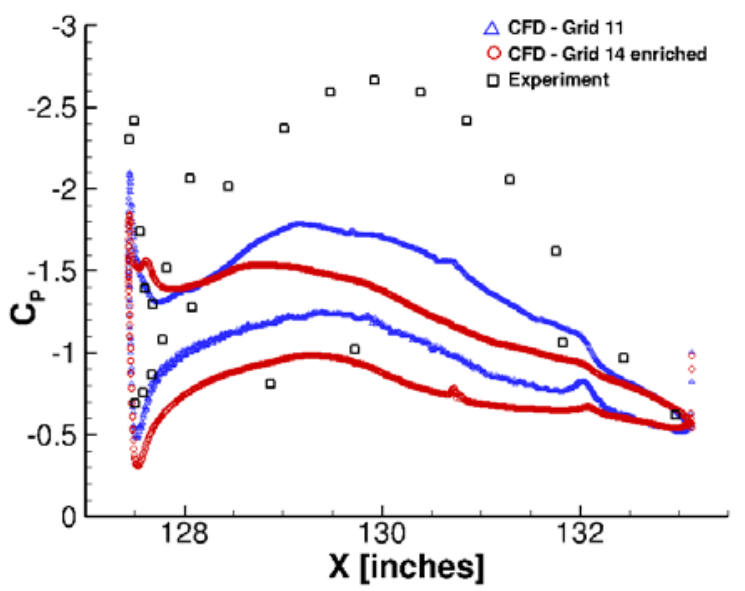

g) Flap row 11

Figure 26. Concluded.

The measured flap pressures revealed the presence of a three-dimensional flow separation zone between flap row 7 and the outboard tip. Most turbulence models cannot predict correctly three-dimensional boundary layer separation on smooth surfaces. Even more challenging are flows subjected to severely adverse pressure gradients and containing confluent boundary layers, which is the case for a highly deflected flap. Therefore, we anticipate that compari- 
sons between computed and measured $\mathrm{Cp}$ values beyond the flap mid-span will not to be as favorable as those observed in the inboard portion of the flap span. Looking at the outboard segment of the flap, the time-averaged Cp values show a more extensive flow separation zone on the flap top surface than indicated by the measured data. However, the most noticeable difference in the pressures occurs at the flap outboard tip (row 11). At this row, the simulated time-averaged Cp values significantly underestimate the broad suction peak that occurs in the mid-chord region; the increased resolution achieved with grid 14-enriched degrades, rather than enhances, the similarity between predicted and measured values. The mid-chord suction peak observed at the outboard tip is the footprint of the primary vortex that forms at the side edge (see Fig. 18). Small changes in the lateral position of the vortex path could have significant effects on the surface imprint of the vortex pressure field and the magnitude of the induced suction peak. Quite possibly, the larger separation zone in the outboard segment of the flap predicted by the simulations affects the loading at the tip, shifting the vortex position laterally. The observed discrepancies near the outboard tip made it imperative to further explore the mesh dependencies of this separation zone and determine whether the time-averaged solution had reached a grid independent state. In fact, resolution of this issue was the main reason for refining the baseline grid 14 near both flap tips. Comparison of the computed flap pressures (in particular rows 8 to 11) from grids 14 and 14-enriched failed to reveal any noticeable improvement in the Cp values. In contrast, the companion computations of Ref. 20, obtained with the PowerFLOW® solver, are in much better agreement with the measured flap pressures using a mesh resolution comparable to that employed in the present study. Furthermore, FUN3D steady results with a pure S-A turbulence model produced better mean Cp distributions near the flap tip than the unsteady hybrid calculations. The sensitivity of the flap tip pressure distributions to the turbulence model, combined with the lack of improvement associated with a locally refined mesh, logically point to limitations in the MDDES approach used here as the main source of the discrepancies in the Cp distributions near the outboard flap tip.

Deployment of the main landing gear only affects the time-averaged pressure field on the bottom surface of the wing (due to the gear cavity opening; row 3 in Fig. 2) and the region in the immediate vicinity of the flap inboard edge. The Cp distributions for the two rows closest to the inboard edge (rows 1 and 2) are shown in Fig. 27. As was the case with the gear off configuration, good agreement between simulated and measured values is obtained. This indicates that gear-flap interaction and its effect on the steady loading at the tip are well predicted. The Cp variations along the circumference of the two wheels are shown in Fig. 28. The computed pressure distributions are in excellent agreement with measured values- the stagnation point and peak suction levels on both wheels are captured well.

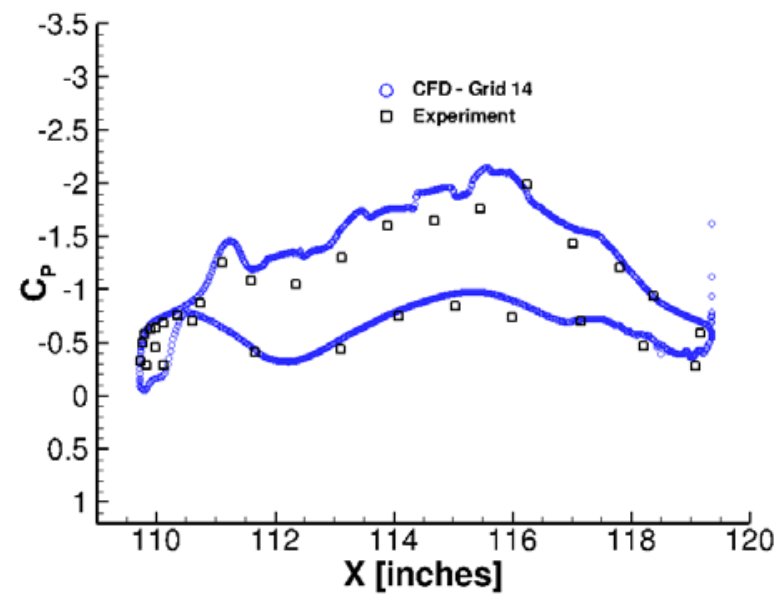

a) Flap row 1

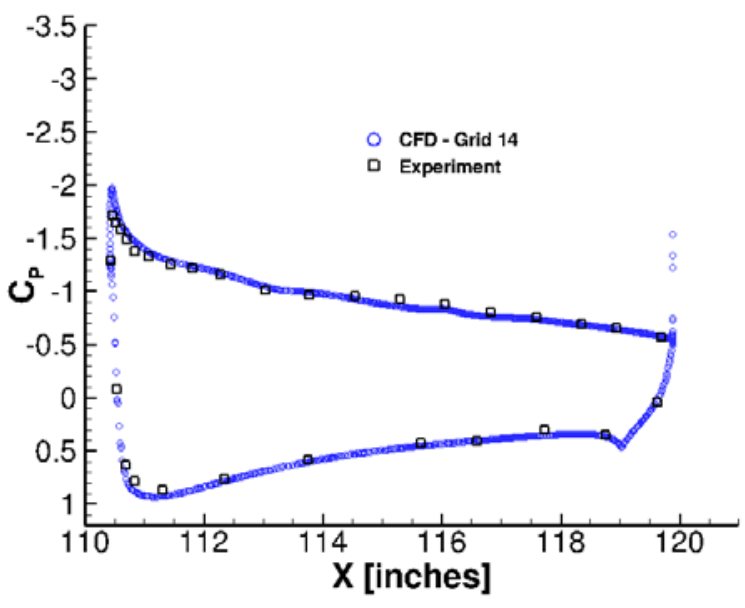

b) Flap row 2

Figure 27. Surface pressures at flap inboard edge for configuration with main gear on. 


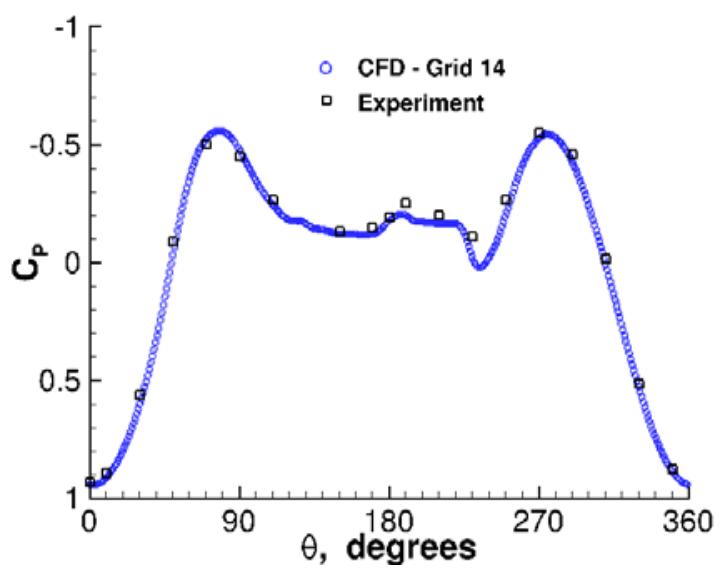

a) Inner wheel

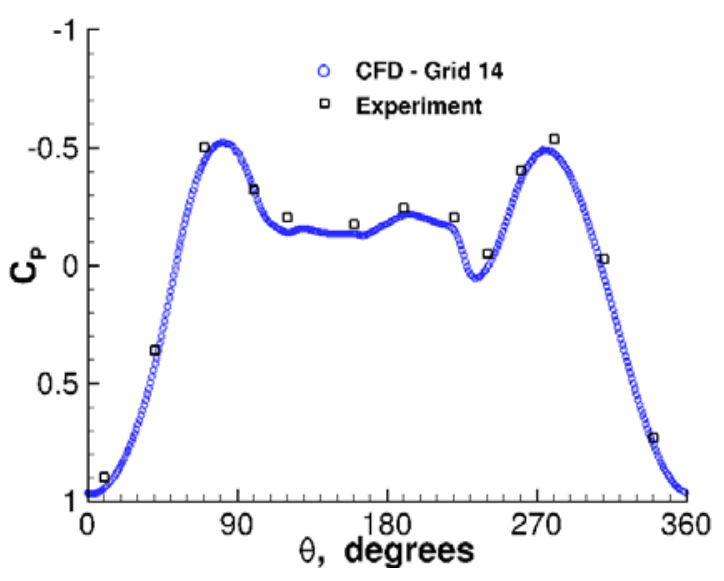

b) Outer wheel

Figure 28. Circumferential pressure distributions on landing gear wheels.

\section{Surface Pressure Fluctuations}

Measured and simulated power spectral density (PSD) values at various probe locations corresponding to the installed pressure sensors at both flap tips are presented in this section. Accurate prediction of the surface pressure fluctuations in the vicinity of the flap side edges is critical for a proper assessment of airframe noise source location. Initial analysis of the unsteady surface pressures indicates that the overall trends are encouraging. Yet, further improvement in some aspects of the computed solutions is still desired. Results from grids 11 and 14-enriched are shown simultaneously in Figs. 29-36 to highlight the fact that attainment of a fully converged, grid-independent solution remains elusive. Unlike the time-averaged Cp results shown in the previous section, refinement of the offsurface grid beneath the flap has a noticeable impact on the surface pressure fluctuations at some locations. The higher resolution imposed under the flap and in the vicinity of the flap tips necessarily results in increased mesh density in the viscous boundary layer also. The added benefit gained from this extra resolution mostly impacts the fluctuation amplitudes in the regions adjacent to the flap leading edge near the tips where the vortices originate and the scale of the critical flow features is very small.

\section{Fluctuations at Inboard Tip}

The pressure fluctuations from the probes placed along the edge on the flap top surface are presented in Fig. 29. At probes 67 and 1, positioned at about 0.25c and 0.40c, respectively (Figs. 29a and Fig. 29b), the effect of grid refinement is quite pronounced. This results in a significant jump in the energy level of the fluctuations for the entire frequency range of interest. For locations along the edge that are farther downstream (probes 3, and 5), grids 11 and 14-enriched produce nearly identical signals. In general, the PSD results obtained with grid 14-enriched over predict the recorded values. The pressure spectra from probes 4,7 , and 9 on the top surface away from the edge are shown in Fig. 30. These sensor positions were specifically chosen to capture the post- merged tip vortex footprint on the flap top surface. For these probes, the computed and measured pressures agree rather well, especially at probe locations 7 and 9, where very good agreement is obtained for frequencies up to about $10 \mathrm{kHz}$. This indicates that grid 11 provides sufficient resolution in the aft section of the flap chord, which is somewhat surprising since the tip vortex path at that location has already detached from the flap top surface.

The pressure spectra from the four probes $(68,10,11$, and 12$)$ installed on the inboard edge side wall are plotted in Fig. 31. Notice that at probes 68 and 10 grid refinement markedly improves the accuracy of the computed pressure fluctuations, capturing the broad tonal hump $(400-1,200 \mathrm{~Hz})$ present in the measured spectra.

The fluctuating pressure field from probes 15 and 16 on the bottom surface of the flap are presented in Fig. 32. As expected, the energy levels on the bottom surface are significantly lower than those recorded on the side wall or on the top surface. Good agreement between the computed and measured PSD values is obtained. That this is so for probe 16 is surprising, since this probe is positioned inward from the bottom edge (see Fig. 4b). Probe 16 is mostly exposed to the passage of a highly skewed accelerating boundary layer drawn to the edge by the presence of a large pressure suction region induced by the tip vortex. Because it employs RANS equations to simulate the flow near solid surfaces, the MDDES approach used in this work is incapable of capturing the development of the fluctuating pressure field associated with an attached turbulent boundary layer. Therefore, the most logical explanation for the 
good agreement seen in Fig. 32b would be that the boundary layer on the flap bottom surface was laminar during the test and the fluctuating pressure field recorded in this area is the imprint of the radiating pressure waves produced at the inboard tip.

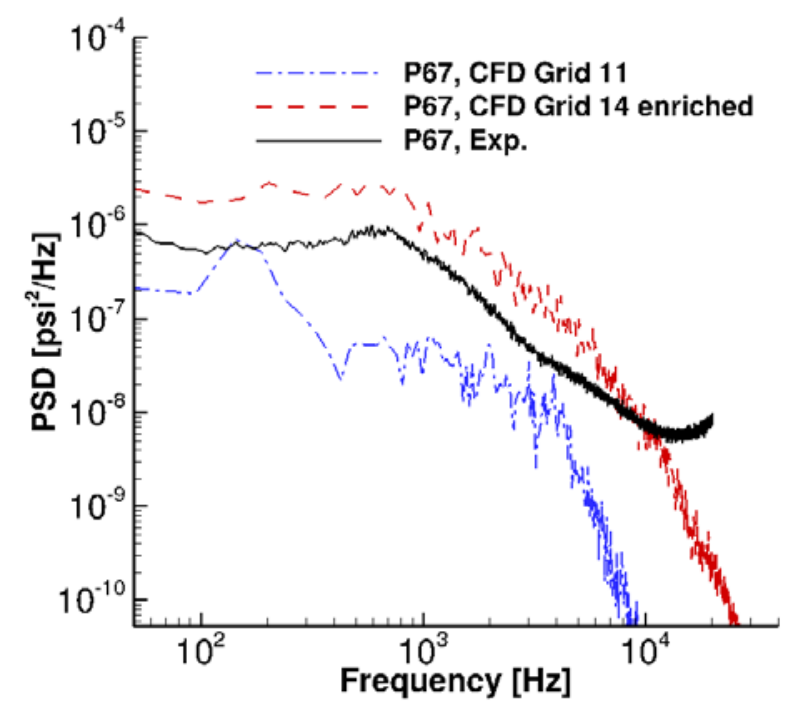

a) Probe 67 location

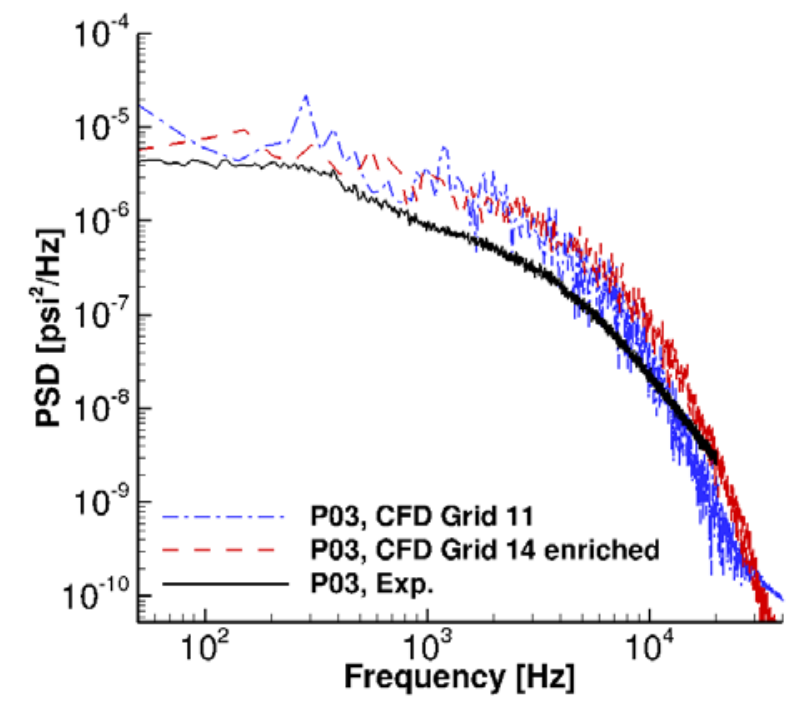

c) Probe 3 location

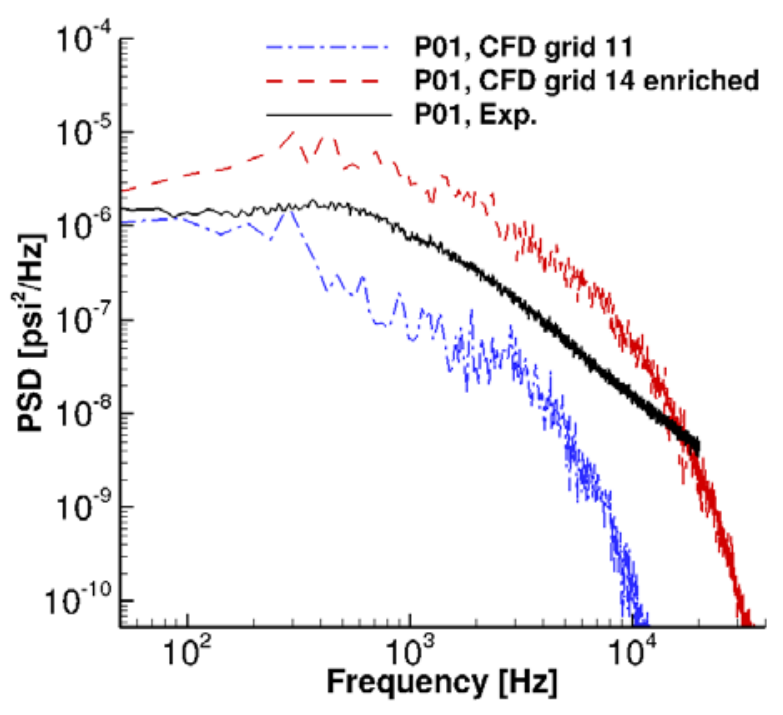

b) Probe 1 location

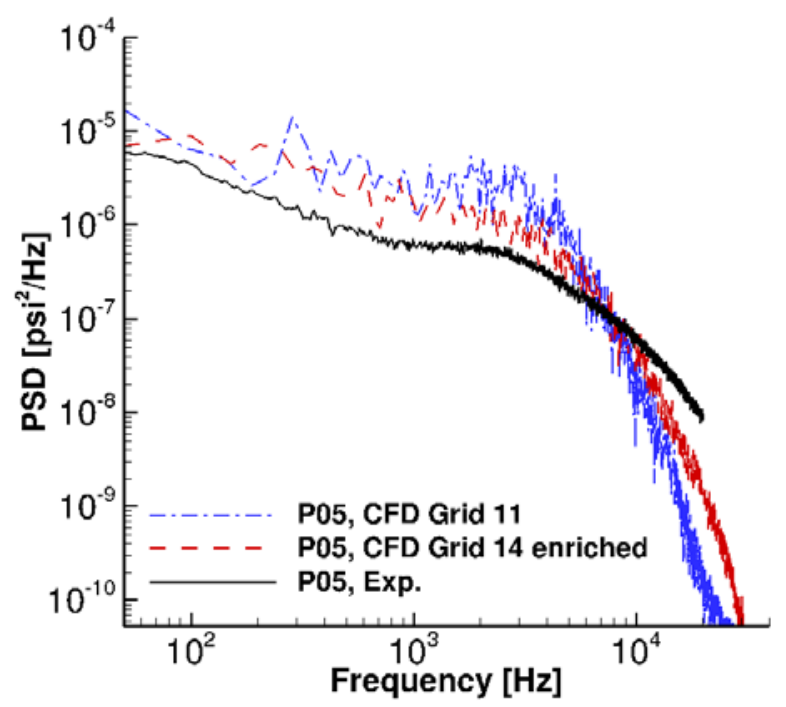

d) Probe 5 location

Figure 29. Power spectral density plots of surface pressure fluctuations at flap inboard tip - top surface edge. 


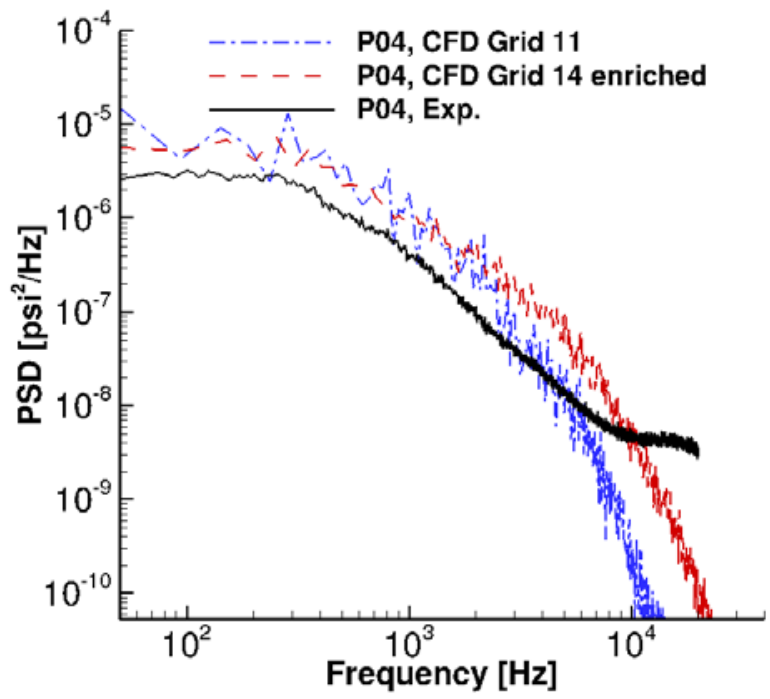

a) Probe 4 location

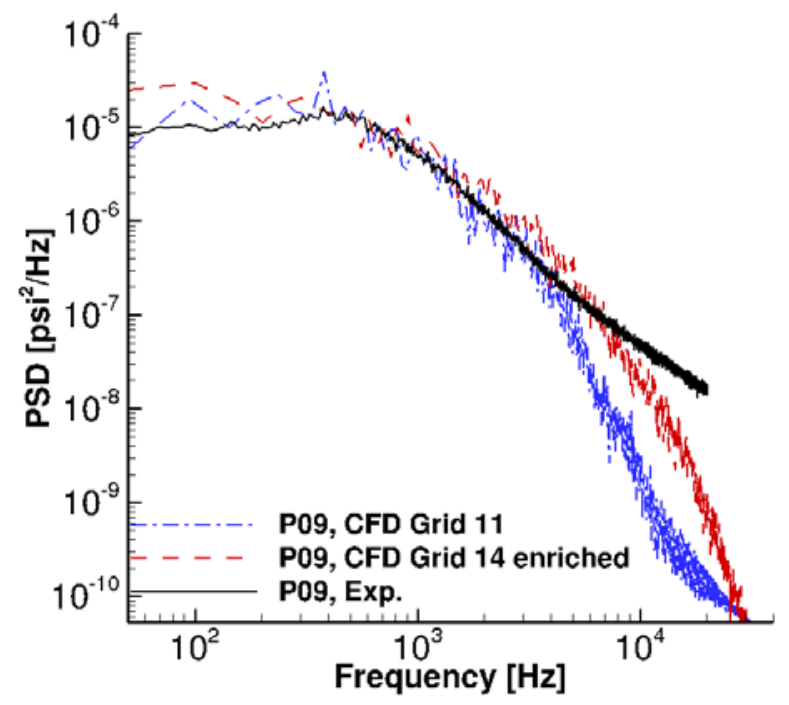

c) Probe 9 location

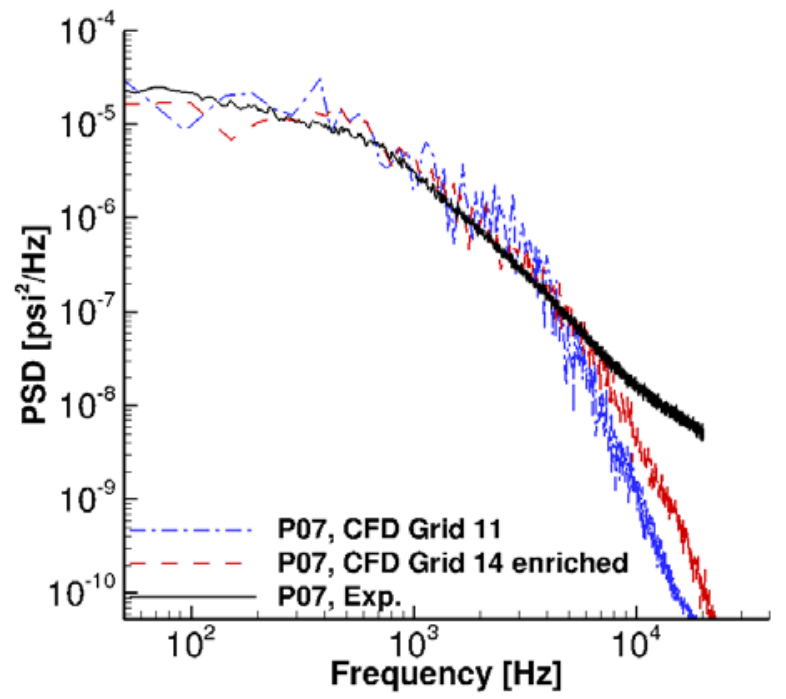

b) Probe 7 location

Figure 30. Power spectral density plots of surface pressure fluctuations at flap inboard tip - top surface away from edge. 


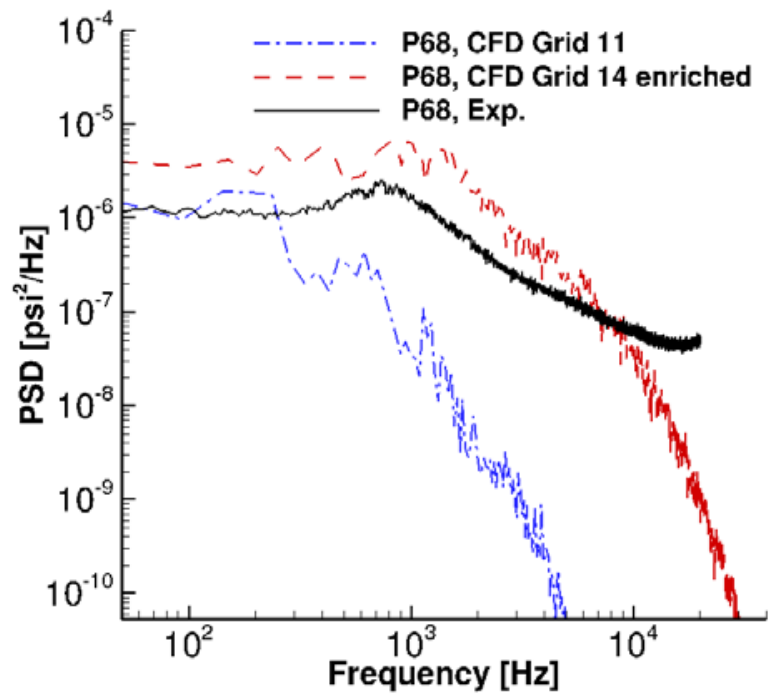

a) Probe 68 location

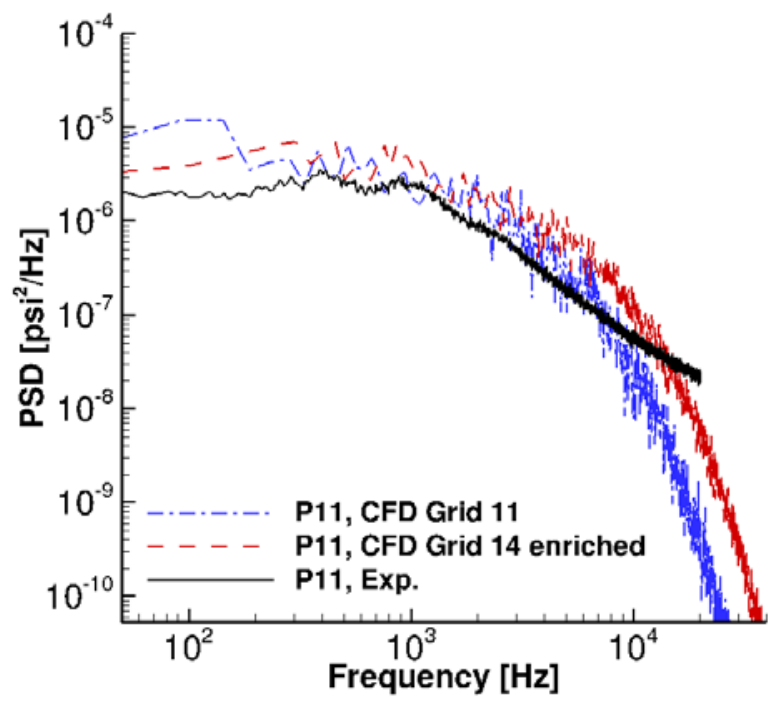

c) Probe 11 location

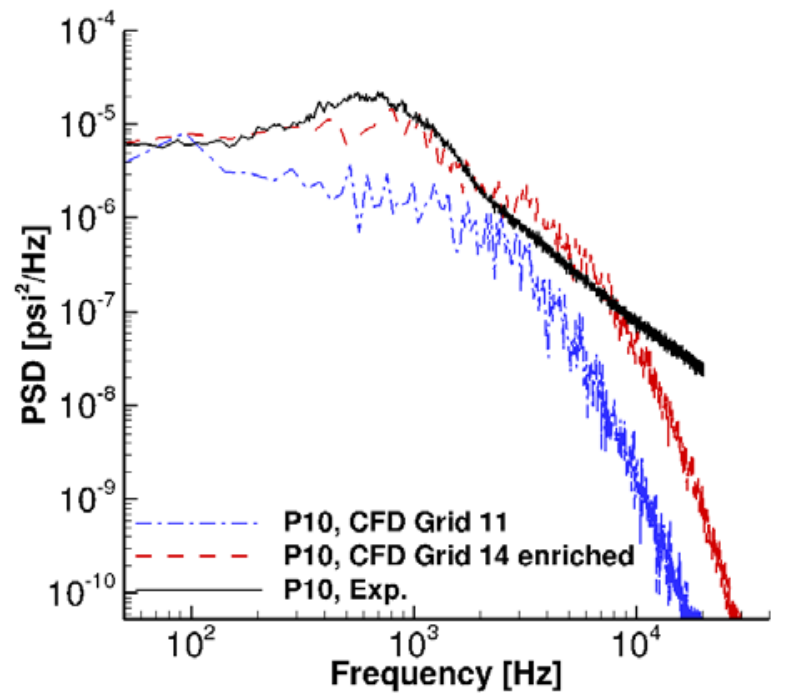

b) Probe 10 location

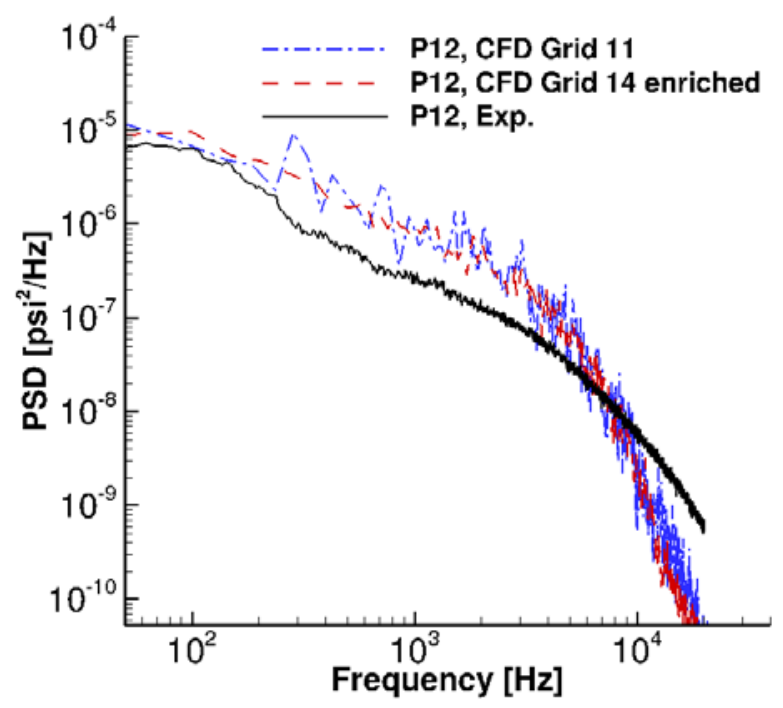

d) Probe 12 location

Figure 31. Power spectral density plots of surface pressure fluctuations at flap inboard tip - edge side wall. 


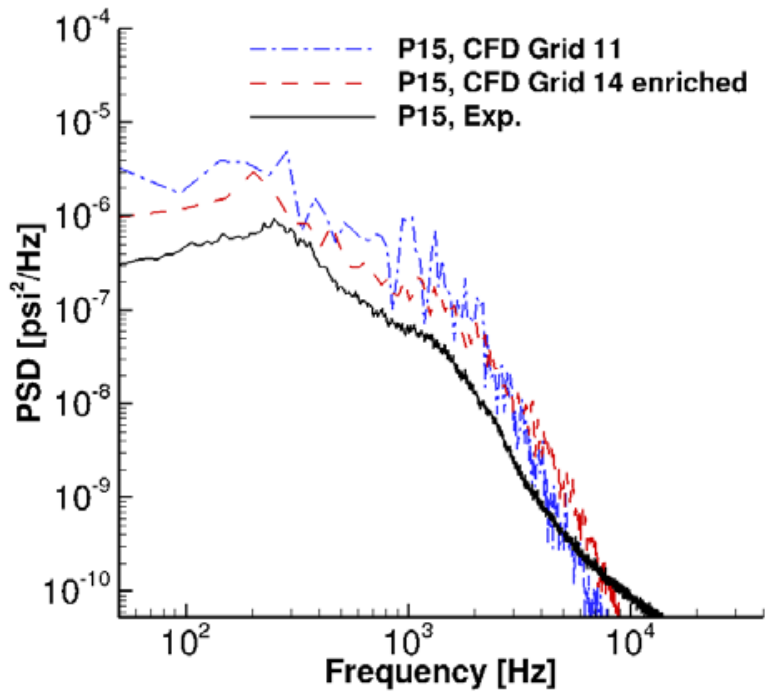

a) Probe 15 location

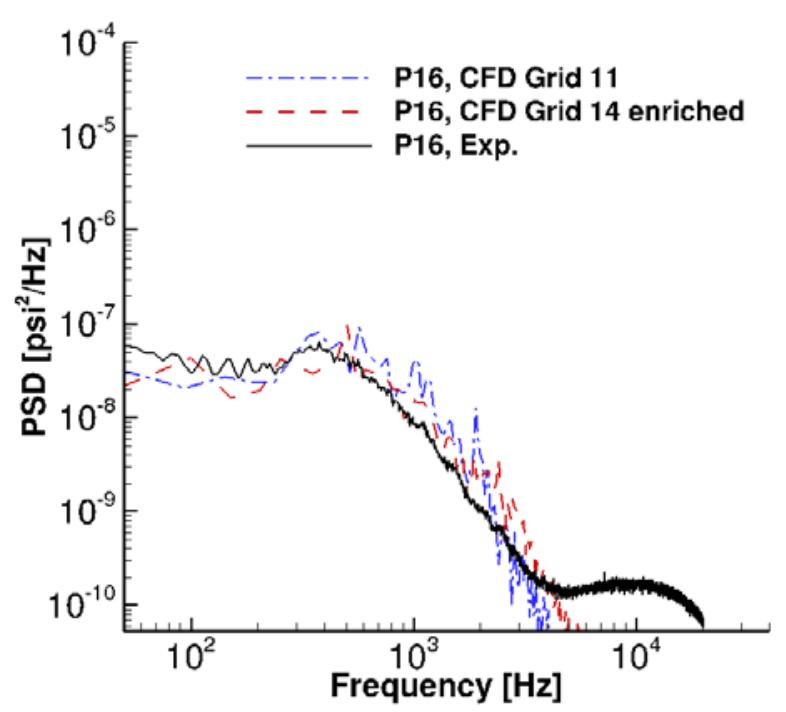

b) Probe 16 location

Figure 32. Power spectral density plots of surface pressure fluctuations at flap inboard tip - bottom surface.

\section{Fluctuations at Outboard Tip}

Prediction of the surface pressure fluctuations at the outboard tip was complicated by the difficulty in producing a realistic geometry of the bulb seal at the outboard tip that closely adhered to what was actually tested. In the $14 \times 22$ wind tunnel tests, the tubular soft foam used to represent the bulb seal was hand glued to the lower interior surface of the cavity at the tip with roughly a third of the tube residing exterior to the cavity; thus, there is a level of uncertainty in correctly duplicating the position of the tubular foam in the simulated model geometry. During testing, the soft foam may have deformed slightly under aerodynamic loads; in contrast, the modeled tubular foam in the simulations was treated as a rigid cylinder. Due to the installed pressure tubes and sensors, the as built/tested tip cavity has many protrusions and depressions on its interior surfaces that are absent in the simulated geometry. Because of these subtle differences, accurately matching the surface pressure fluctuations at discrete locations near the outboard tip is problematic at best. The critical parameter used to judge the success of the simulations is how well the farfield acoustic behavior is predicted. As will be shown in the following section, despite differences in the fluctuating surface pressures, very good agreement between the simulated and measured farfield noise spectra was achieved.

Pressure fluctuations from various probes placed along the edge on the flap top surface are presented in Fig. 33. Except near the leading edge (probe 65), there is good agreement between the spectra for grids 11 and 14-enriched, indicating that the additional resolution in the vicinity of the outboard tip is not providing significantly better results. In general, reasonably good agreement between computed and measured spectra is attained along the upper edge. At probe 65, given the small features of the vortical flow field that are initiated near the leading edge, the finer resolution of grid 14-enriched improves the high frequency content of the simulated spectrum and produces a better match to the measured data. At this location, the enriched grid captures the tonal peak (although at a slightly lower frequency) in the measured spectrum close to 6,500 Hz. Similar tonal behavior was observed in the farfield acoustic measurements of the full scale aircraft during the 2006 flight test (Ref. 6). The tonal feature at full scale was attributed to the flow-cavity interaction at the outboard tip. The surface pressure measurements from the $18 \%$ scale aircraft model and the companion simulations corroborate that conjecture. At probe locations farther downstream along the edge (probes 25, 28, and 66), the comparison between predicted and measured spectra improves. At these positions, the high frequency tone disappears, and a lower frequency tonal feature with its peak near $2,000 \mathrm{~Hz}$ becomes apparent. The tones at $6,500 \mathrm{~Hz}$ and $2,000 \mathrm{~Hz}$ could be related to the same flow features; if so, the reduction in frequency may be caused by the chordwise growth of the side edge vortices. The simulated PSD curves fail to capture the tonal feature present at these positions. For all probe locations, while showing the correct trend, the computed PSD levels significantly over-predict the measured values in the sub $300 \mathrm{~Hz}$ frequency range. This divergence is attributed to 1) the presence of a large flow separation zone near the outboard tip that produces high amplitude low frequency oscillations and 2) the relatively short record length of the simulated signal. 


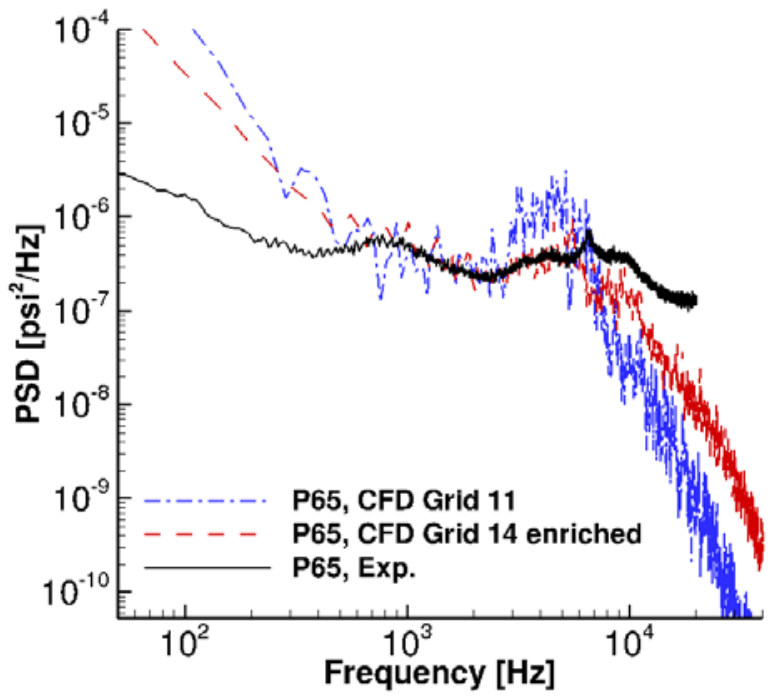

a) Probe 65 location

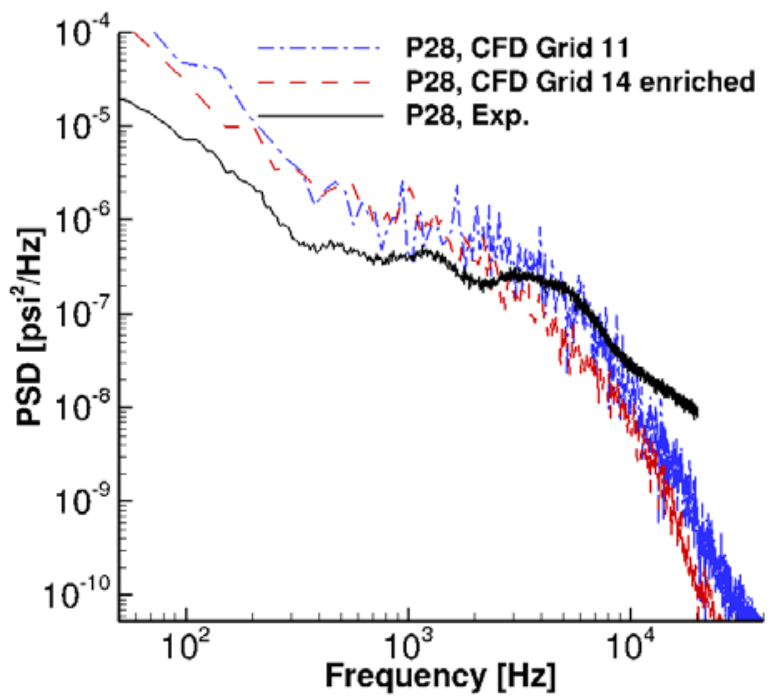

c) Probe 28 location

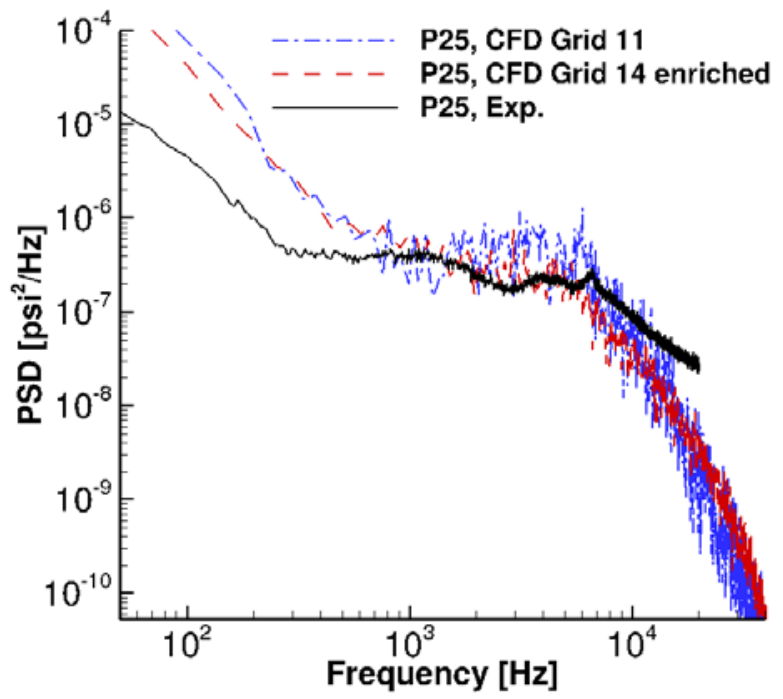

b) Probe 25 location

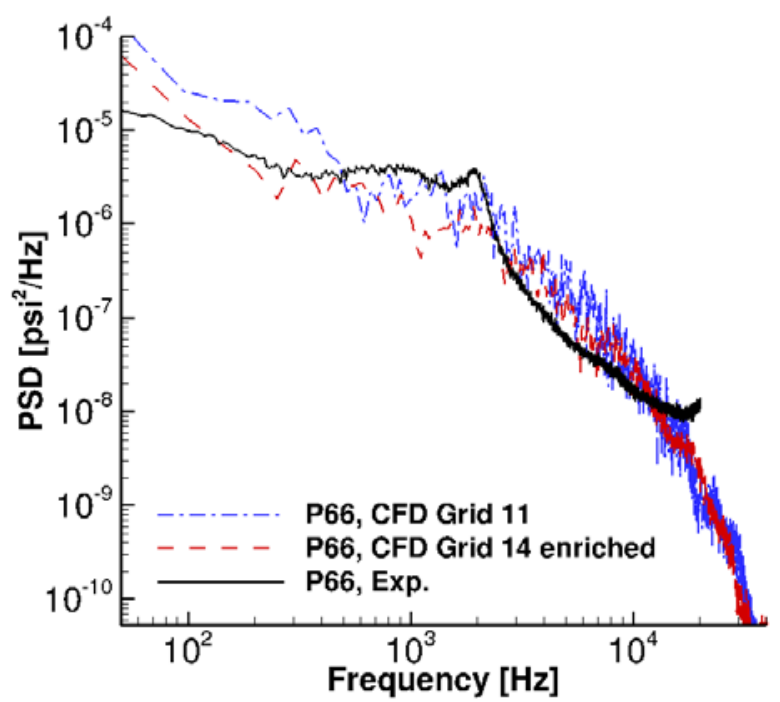

d) Probe 66 location

Figure 33. Power spectral density plots of surface pressure fluctuations at flap outboard tip - top surface edge.

Pressure spectra from probes 29 and 30 on the top surface away from the edge are shown in Fig. 34 . As was the case for the inboard tip, these sensor positions were selected to extract the post- merged footprint of the tip vortex on the flap upper surface. At both locations, the simulated PSDs significantly over predict the measured levels. Since the results obtained with grid 11 and grid 14-enriched are very similar, the over prediction cannot be attributed to the lack of resolution. Other factors, such as the role of the turbulence model or the MDDES methodology employed, must be explored. The computed spectra from the two probes inside the tip cavity (probes 26 and 27) are shown in Fig. 35. At the location of probe 26, that is, close to the flap leading edge, the simulated spectrum is very different in shape and magnitude than the measured spectrum. For frequencies above $400 \mathrm{~Hz}$, better agreement between the computed and measured spectra is obtained for the second probe in the cavity (probe 27), which is located about 0.60c from the leading edge. The comparison for the spectra obtained from probes on the flap bottom surface (probes 32 through 35) is shown in Fig. 36. As in the other PSD plots, the simulated results are of mixed quality for mid to high frequencies, good agreement is observed at some probe locations (probes 32 and 35) while significant differences exist at other probe positions (33 and 34). In general, the fluctuation levels on the bottom surface are one or two orders of magnitude lower than those observed on the top surface. 


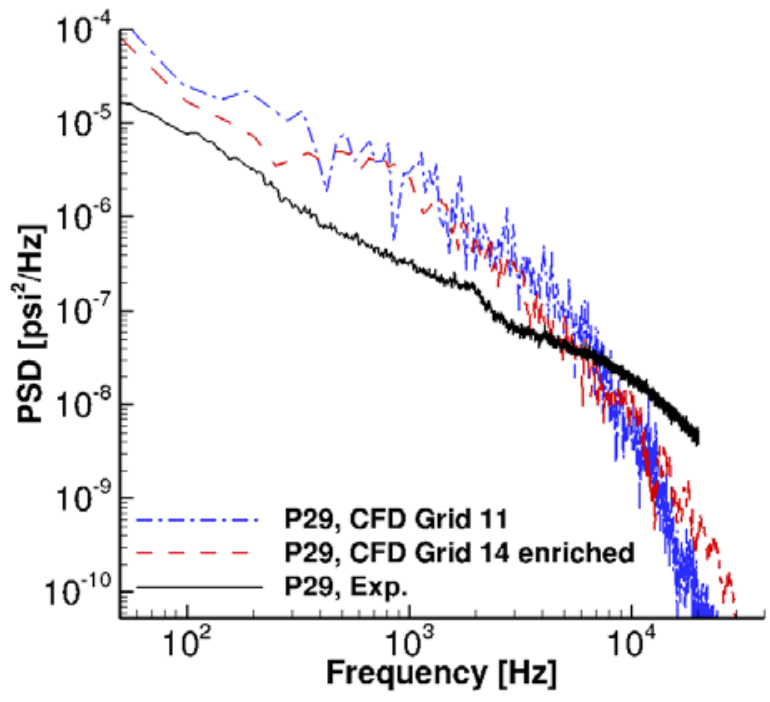

a) Probe 29 location

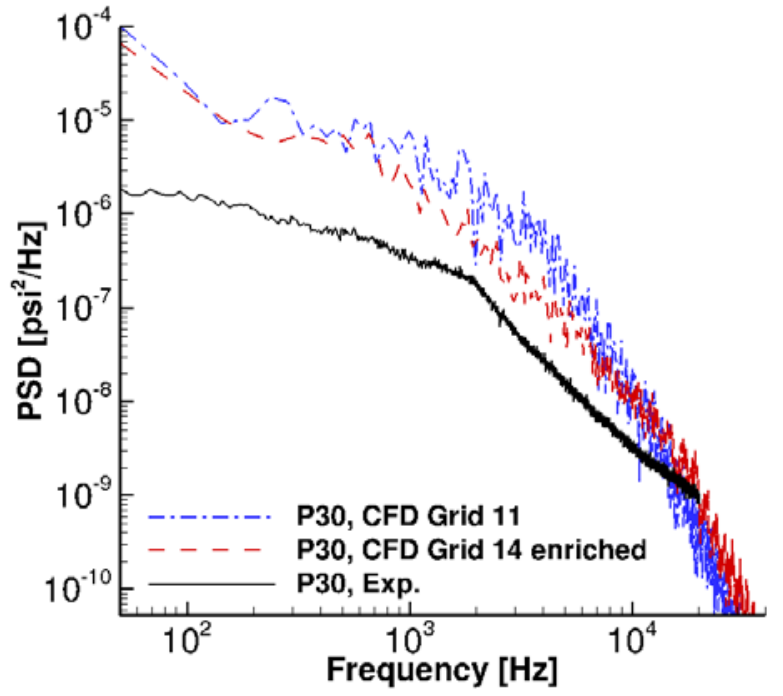

b) Probe 30 location

Figure 34. Power spectral density plots of surface pressure fluctuations at flap outboard tip - top surface away from edge.

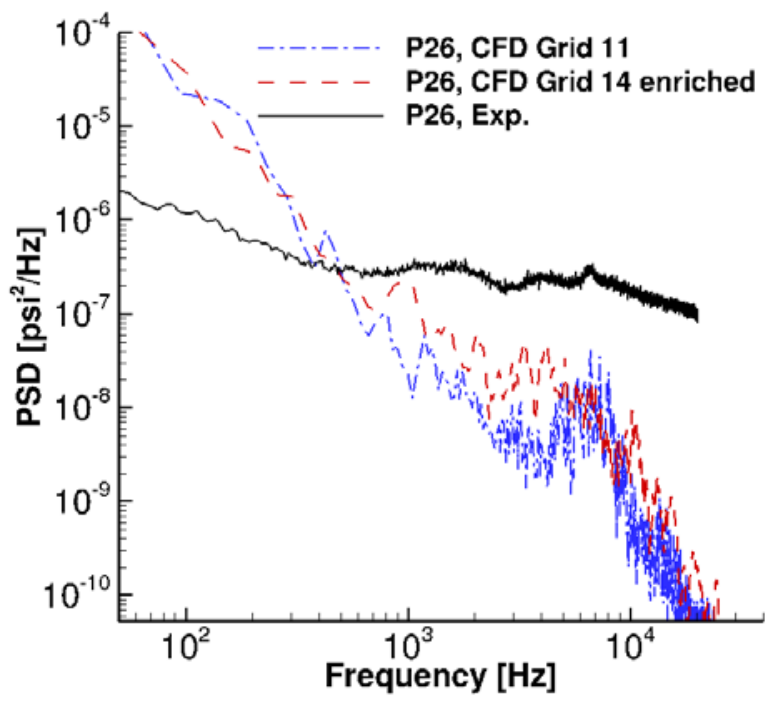

a) Probe 26 location

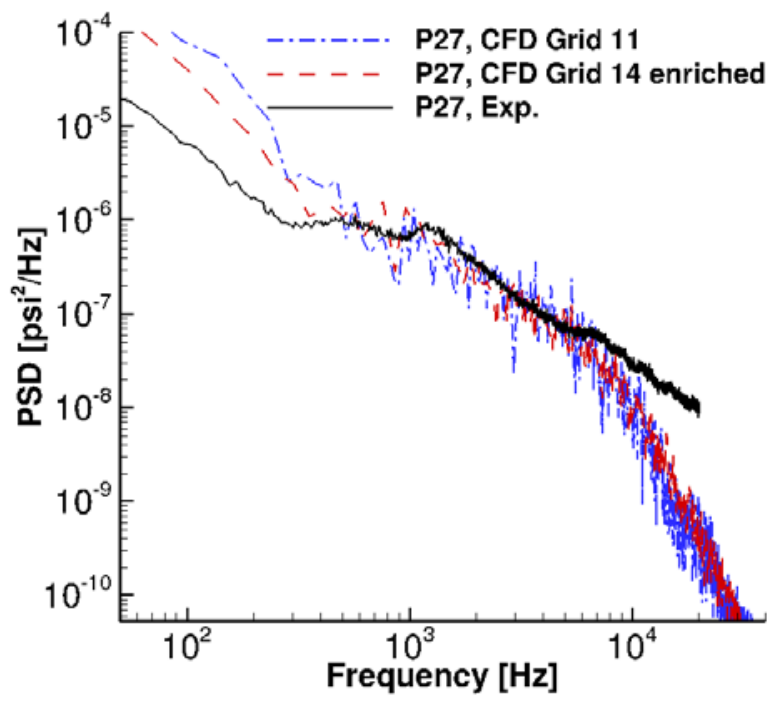

b) Probe 27 location

Figure 35. Power spectral density plots of surface pressure fluctuations at flap outboard tip - tip cavity.

Overall, the FUN3D predicted pressure spectra at both inboard and outboard flap tips are in fair agreement with the measured PSDs. However, the corresponding PSD results obtained with a Lattice Boltzmann approach, presented in Khorrami et al. ${ }^{20}$, show excellent agreement with the measured spectra. Since both studies used the same $18 \%$ scale aircraft model and comparable mesh resolution at the flap tips, geometric fidelity and grid density effects cannot be the reasons for the decreased accuracy of the FUN3D-generated steady Cp and surface pressure spectra at the outboard tip. As stated earlier, the most likely sources of the observed discrepancies in the near field comparisons are inherent limitations in the MDDES methodology employed in the present simulations. 


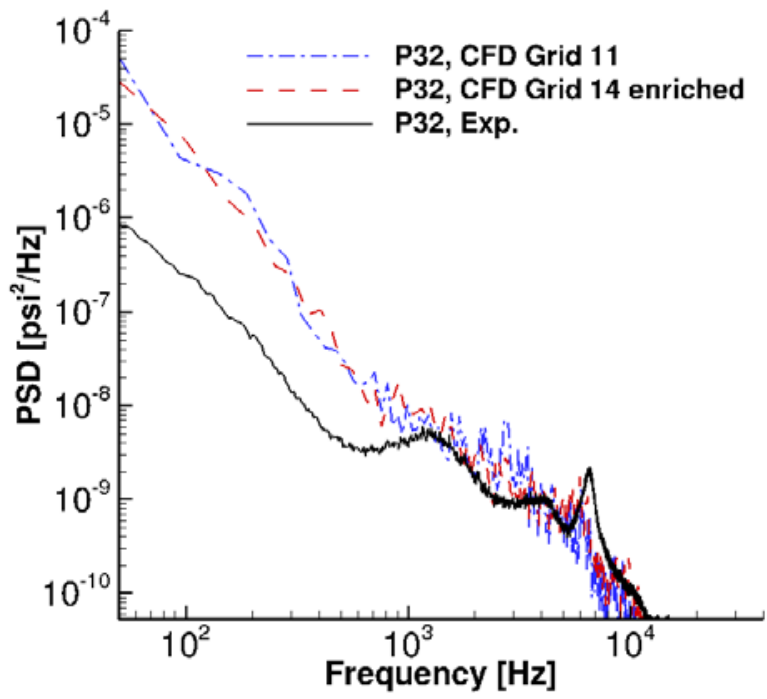

a) Probe 32 location

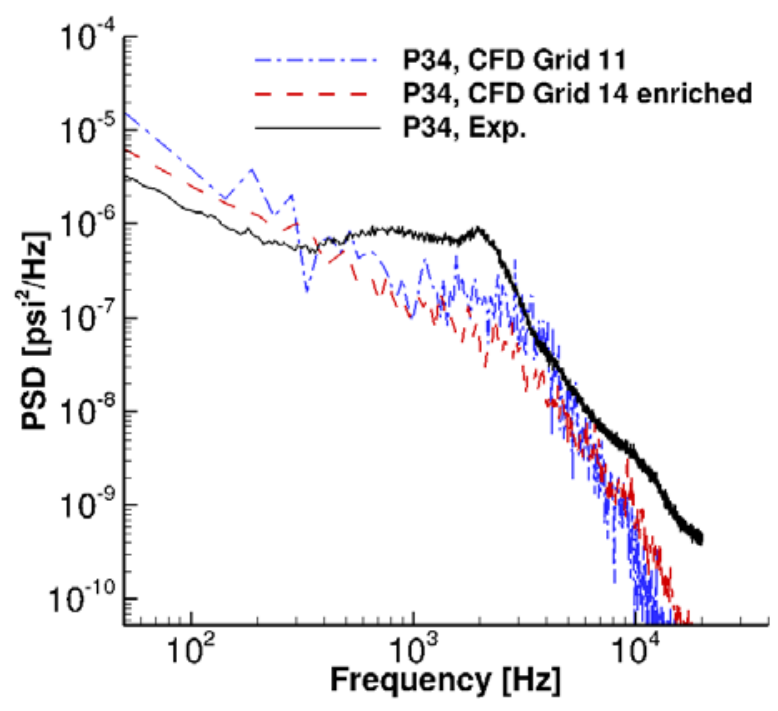

c) Probe 34 location

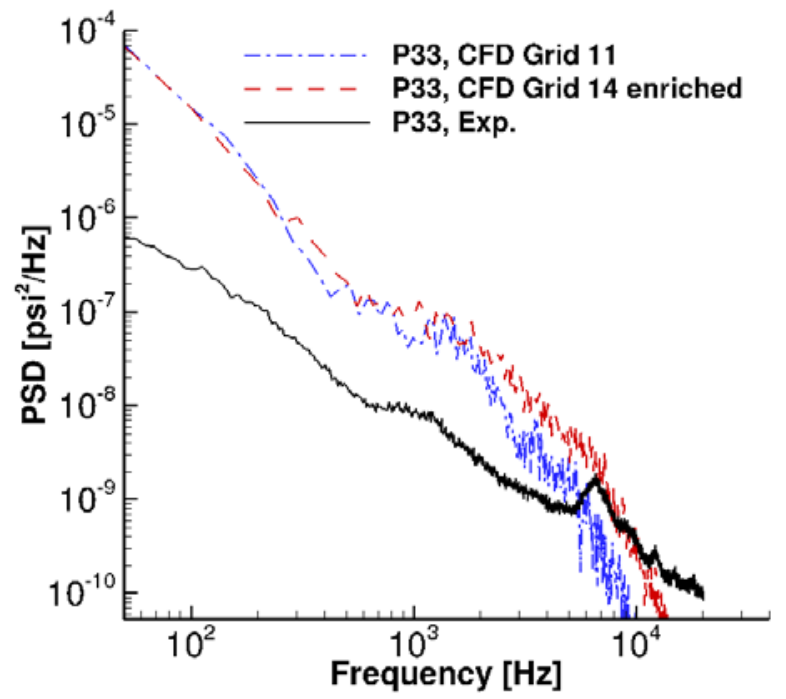

b) Probe 33 location

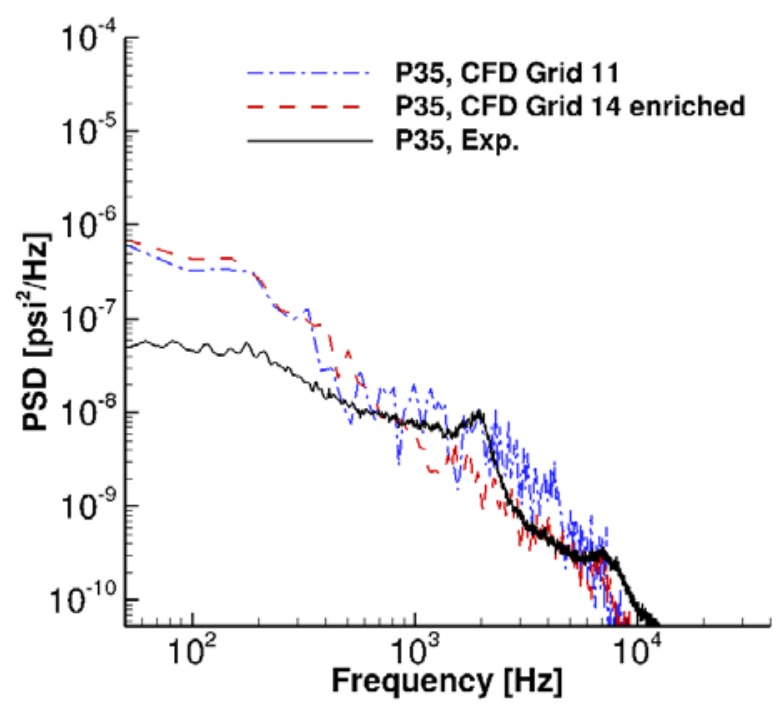

d) Probe 35 location

Figure 36. Power spectral density plots of surface pressure fluctuations at flap outboard tip - bottom surface.

\section{Main Landing Gear}

The gear-flap interaction as it affects the fluctuating pressure field at the flap inboard tip is examined next. We will probe a select number of locations where noticeable changes in the measured values were shown to occur in Ref. 10. The PSD comparison at various probe locations on the upper surface and side wall at the flap inboard tip are presented in Fig. 37. The predicted spectra are in much better agreement with the measurement data, as compared to the gear off configuration. Thus, gear-flap interaction effects on fluctuating surface pressure are reasonably well captured by the simulations. This is unexpected, since the deployed main gear significantly increases the complexity of the flow field to be resolved and the demand for accuracy (resolution) in order to maintain the main gear wake intact until it interacts with the inboard tip.

Because of the bluff body nature of the various gear subcomponents, prediction of the measured surface pressure spectra at discrete locations is problematic at best. For example, the presence of a slightest flow angularity in the tunnel freestream velocity has the potential to cause a significant shift in the location of separation/reattachment points. Sample computed surface pressure spectra at select locations on the gear primary subcomponents (struts, wheels, and door) are presented next. 


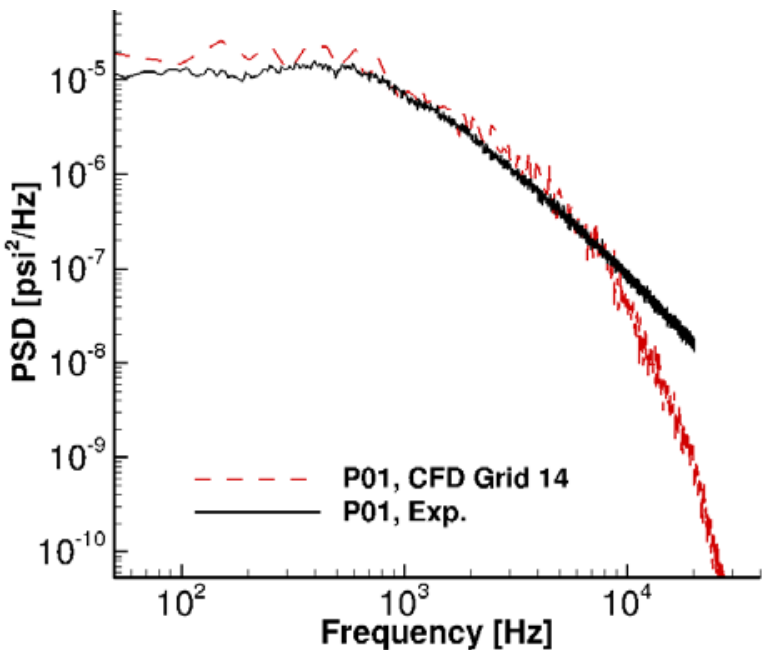

a) Probe 1 location

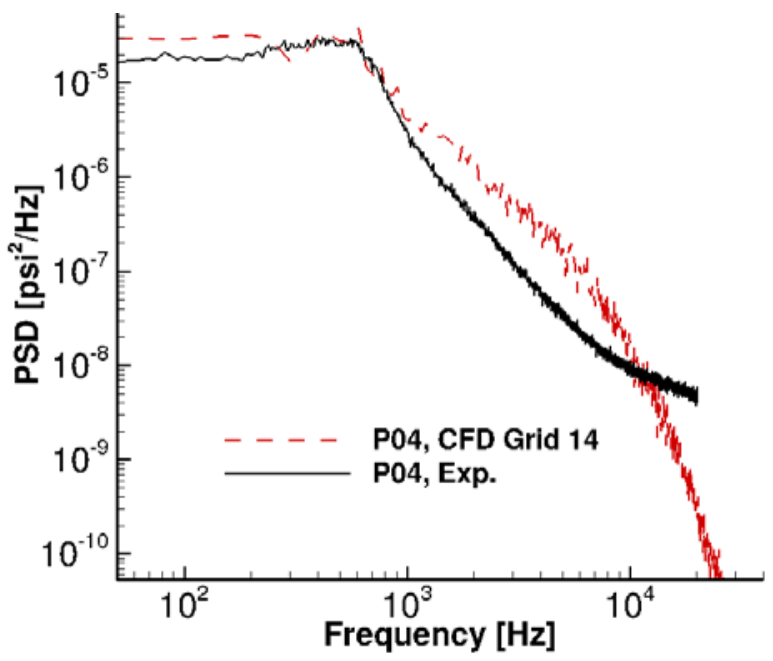

c) Probe 4 location

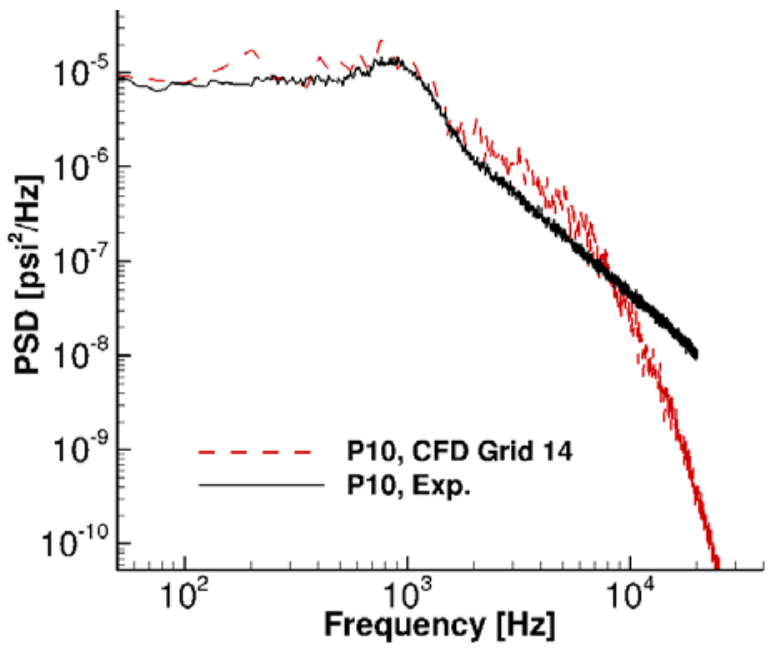

e) Probe 10 location

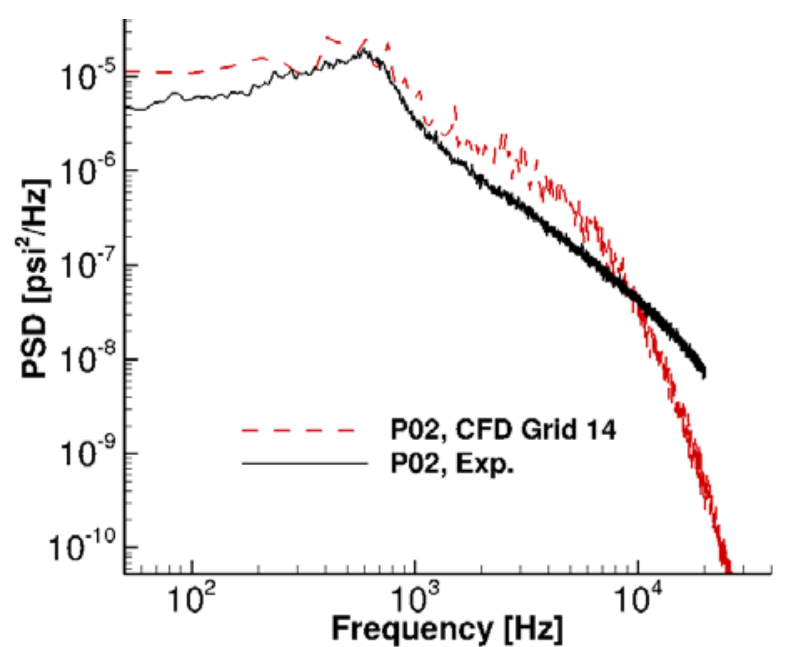

b) Probe 2 location

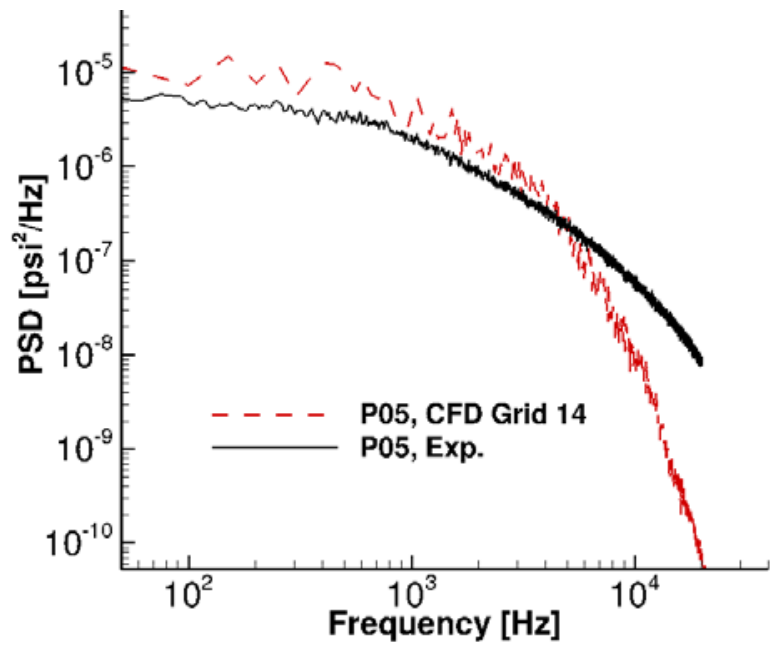

d) Probe 5 location

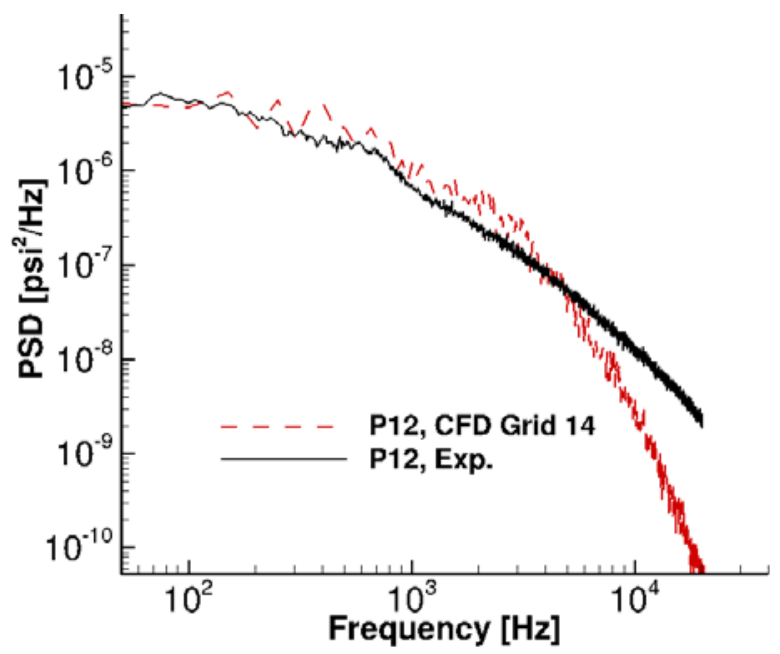

f) Probe 12 location

Figure 37. Power spectral density of surface pressure fluctuations at flap inboard tip with main gear on. 
Measured and simulated surface pressure spectra for probes located on the front (36 and 37, see Fig. 8) and rear struts (39 and 40) are shown in Fig. 38. These probes were placed at strategic positions to capture flow separation from the gear main post and the impact of the free shear layer on the rear strut. Except for the PSD values associated with probe 37, the computed spectra are in reasonable agreement with the experimental data. The measured spectrum at probe 37 has a broad tonal feature with a peak frequency centered around $700 \mathrm{~Hz}$. Based on the physical dimension of the main post, most likely this tone represents mild vortex shedding from the post. In general, the predicted spectrum significantly under-estimates the energy level at all frequencies and fails to capture the tonal component of the measured spectrum. As mentioned earlier, the slightest movement of the separation point on a bluff body (due to any reason) could have a profound effect on the shape and amplitude of the pressure fluctuation spectra. Recall that the ultimate test for the simulations is the accuracy of the farfield noise prediction.

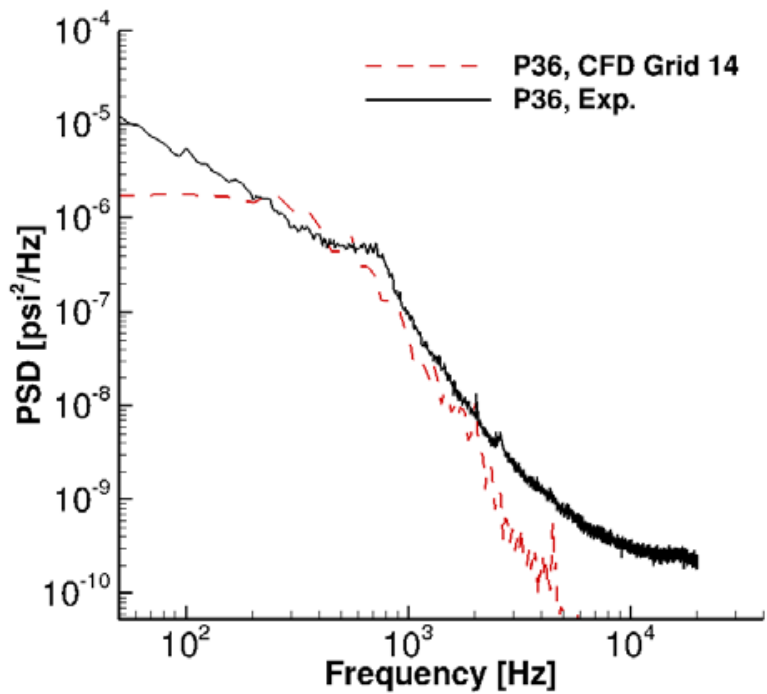

a) Probe 36 location

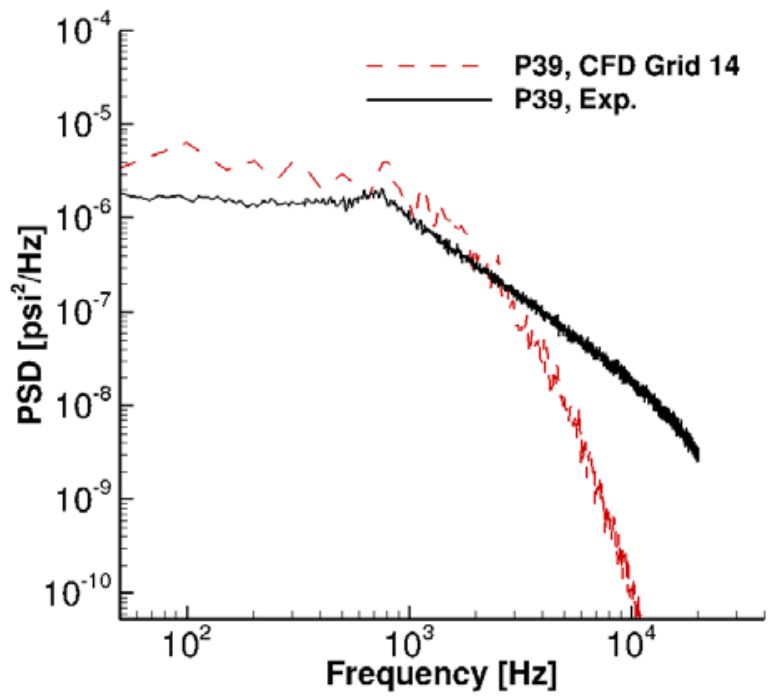

c) Probe 39 location

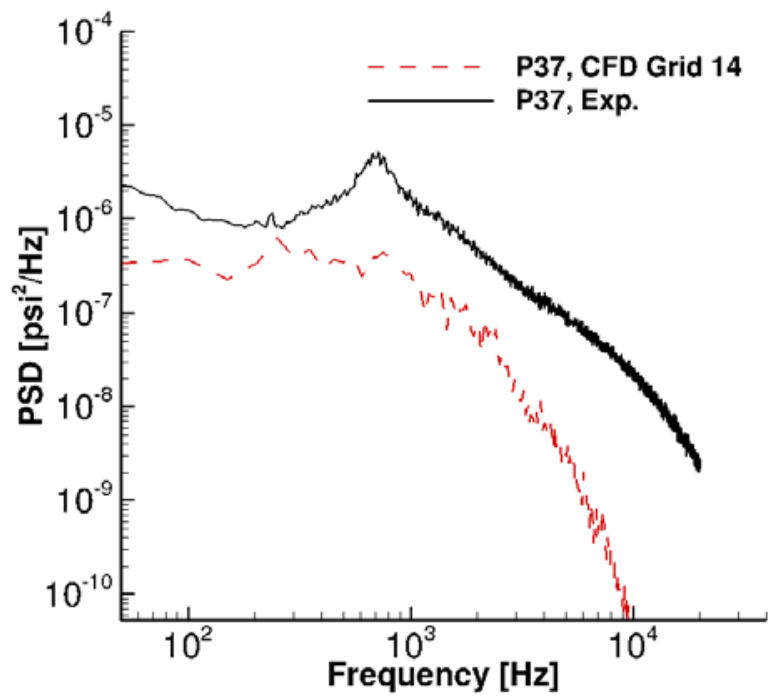

b) Probe 37 location

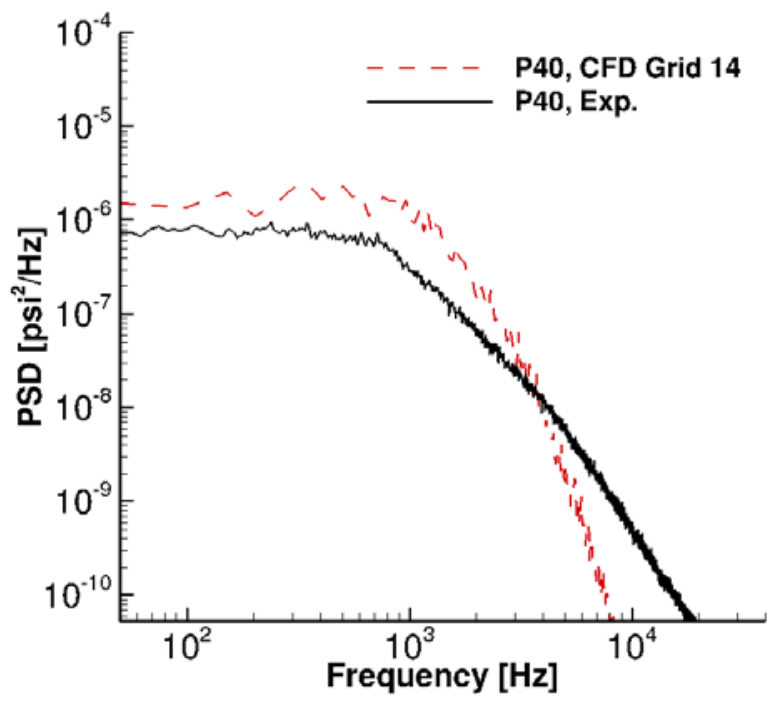

d) Probe 40 location

Figure 38. Power spectral density of surface pressure fluctuations on main gear front (a) and (b), and rear (c) and (d) struts.

Sample PSD plots for probes on the gear inner wheel (43 through 45) are presented in Fig. 39. The predicted spectra follow the measured data in the low to mid frequency range. At frequencies above $2 \mathrm{kHz}$ to $3 \mathrm{kHz}$, there is a rapid roll off (decay) of the spectral levels in the simulated curves. 
Surface pressure fluctuation spectra at two locations on the gear door are shown in Fig. 40. The spectra at the selected probes are representative of those observed at other door locations. Notice that the pressure levels on the door are nearly two orders of magnitude lower than those on the other gear components. The simulated PSD curves capture the frequency content and the fluctuation levels up to frequencies approaching $3 \mathrm{kHz}$. Overall it appears that, for this aircraft configuration, the gear door is not a major noise source and most likely it acts as a reflector for the acoustic waves emanating from the flap inboard tip and/or the other components of the gear.

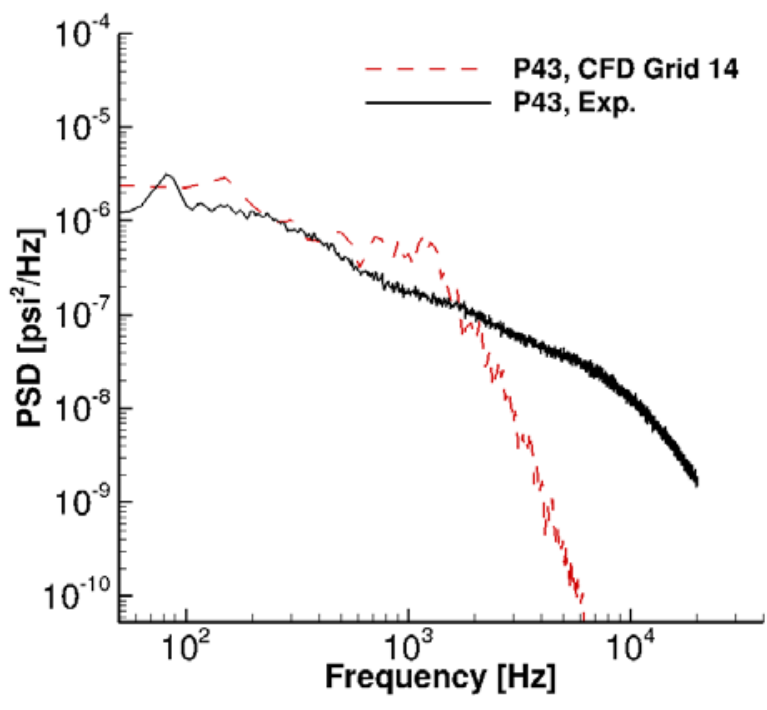

a) Probe 43 location

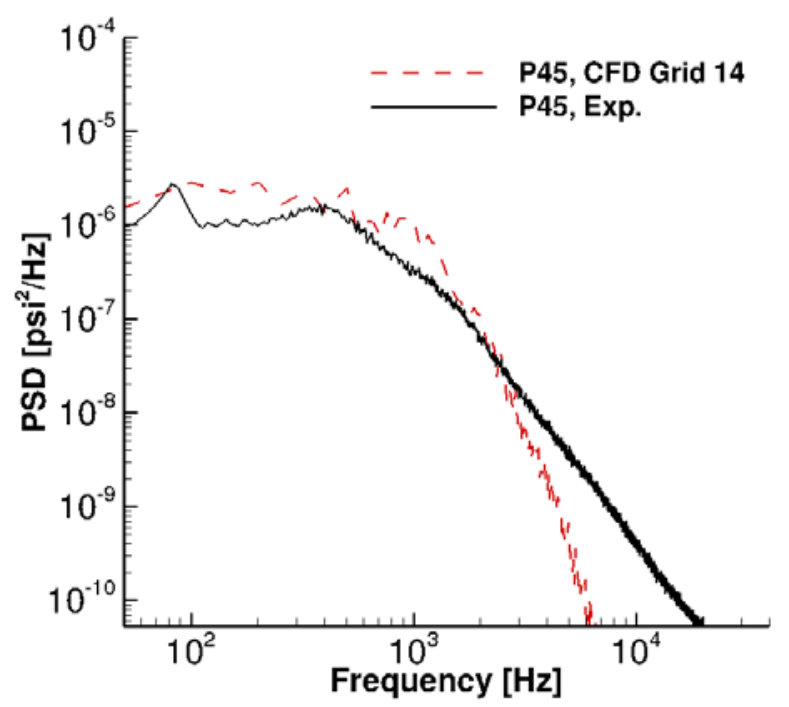

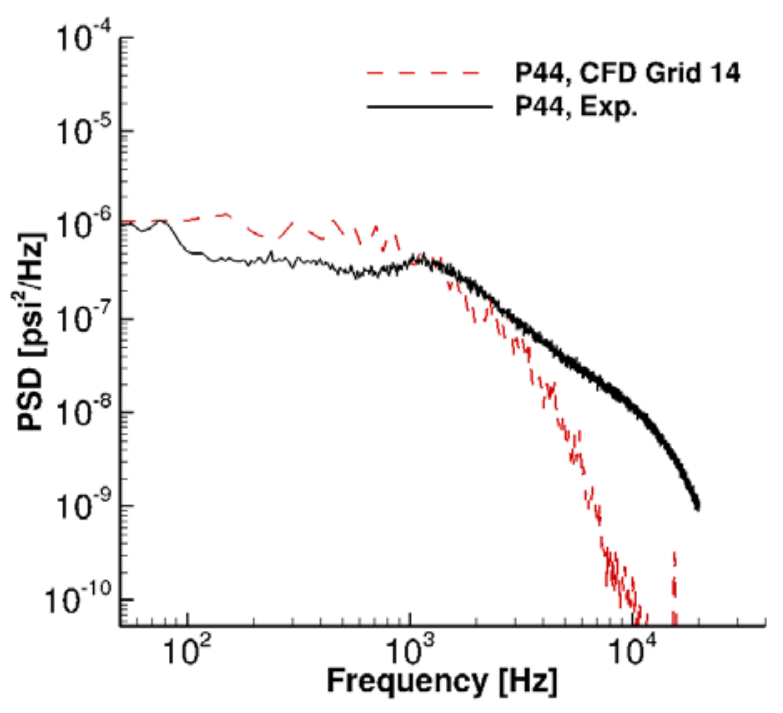

b) Probe 44 location

c) Probe 45 location

Figure 39. Power spectral density of surface pressure fluctuations on main gear inner wheel. 


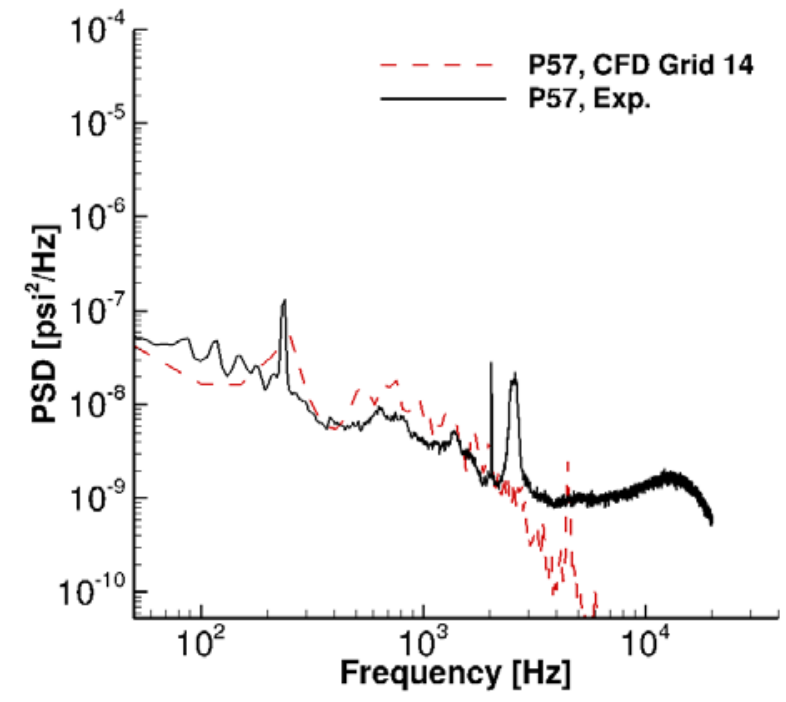

a) Probe 57 location

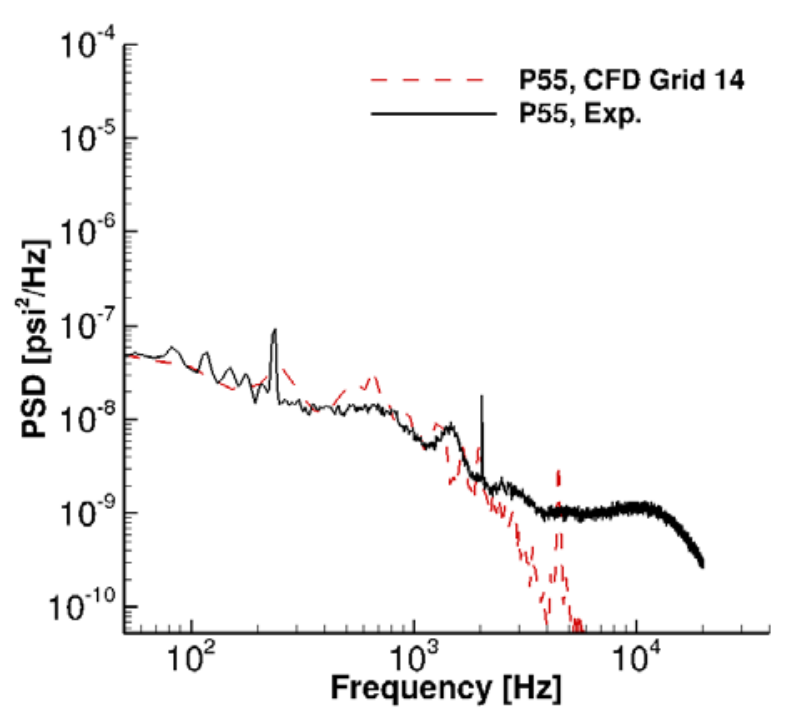

b) Probe 55 location

Figure 40. Power spectral density of surface pressure fluctuations on main gear door.

\section{Near and Farfield Acoustics}

Assessment of the near field acoustic behavior is a crucial step in gaining better insight on the propagating sound waves in the vicinity of the flap inboard and outboard tips, as well as in ensuring that the farfield acoustic propagation is executed properly.

\section{Near Field Noise}

The acoustic signals in the near field were obtained directly from the FUN3D solver output for the baseline configuration without the main gear. The time-dependent pressure records were extracted from the unsteady computations at a series of pre-selected mesh locations (hereafter referred to as CFD probes). Choosing the mid-chord at the flap tips as the origins, CFD probes were positioned along spherical rays that are $20^{\circ}$ apart in both the flyover (x-z) and sideline (y-z) planes. The selected arrangement of the CFD probes at the flap inboard tip is shown in Fig. 41. The corresponding probe distributions at the outboard tip are presented in Fig. 42. Notice from the figures that the successive arcs depicting constant radii are separated by half the local tip chord length, c. Also note that the flyover and sideline planes intersect at $\theta=\varphi=90^{\circ}$, making the ray containing the probes in the overhead direction common to both sets.

Sample PSD plots of the collected pressure signals at the inboard tip are presented in Fig. 43a. Only results from the overhead directivity angle are shown. The PSD values have been multiplied (normalized) by the square of the non-dimensional distance $(\mathrm{r} / \mathrm{c})$ from the origin. A good collapse of PSD values for frequencies above $200 \mathrm{~Hz}$ and distances of up to $\mathrm{r} / \mathrm{c}=2.5$ is observed. Similar trends were also observed for all other directions examined. This indicates that an acoustic field with spherical spreading is established within a relatively short distance from the flap tip. The spectra display a high frequency roll-off that is typical of most turbulent shear flows. The faster decay in the high frequency content for probes farther away is a direct consequence of the drop in spatial resolution of the CFD domain as the distance from the flap tip is increased. For frequencies below $200 \mathrm{~Hz}$ an opposite trend is observed. Due to the relatively long wavelengths, the acoustic waves display a $1 / \mathrm{r}$ decay behavior at larger distances from the flap tip.

The corresponding PSD values obtained from the probes located at the outboard tip are plotted in Fig. 43b. Overall, the PSD values show a behavior similar to that observed for the inboard tip. However, for frequencies above $4 \mathrm{kHz}$, these spectra show much higher levels relative to signals at the inboard tip. 


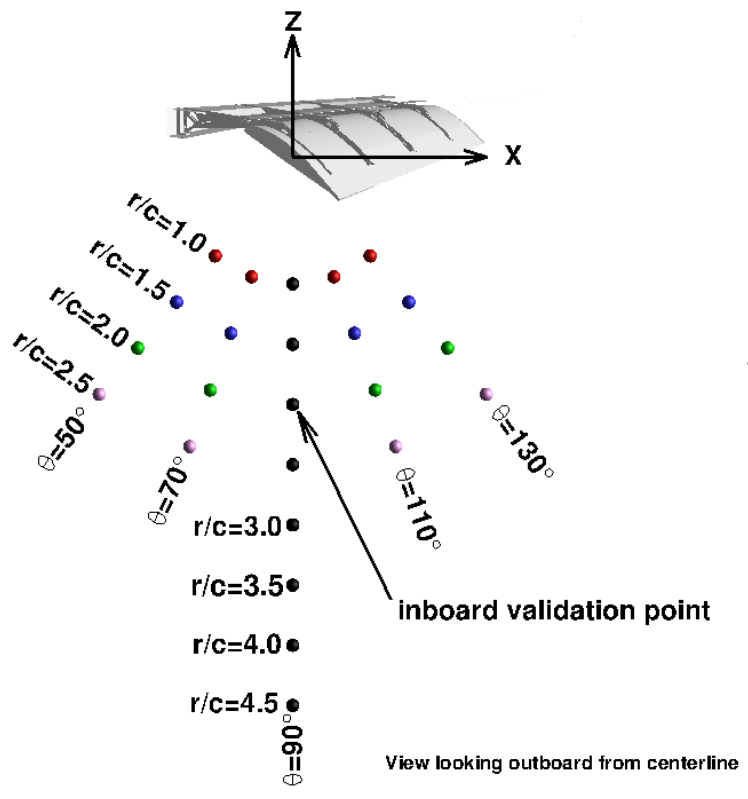

a) Flyover (streamwise) direction

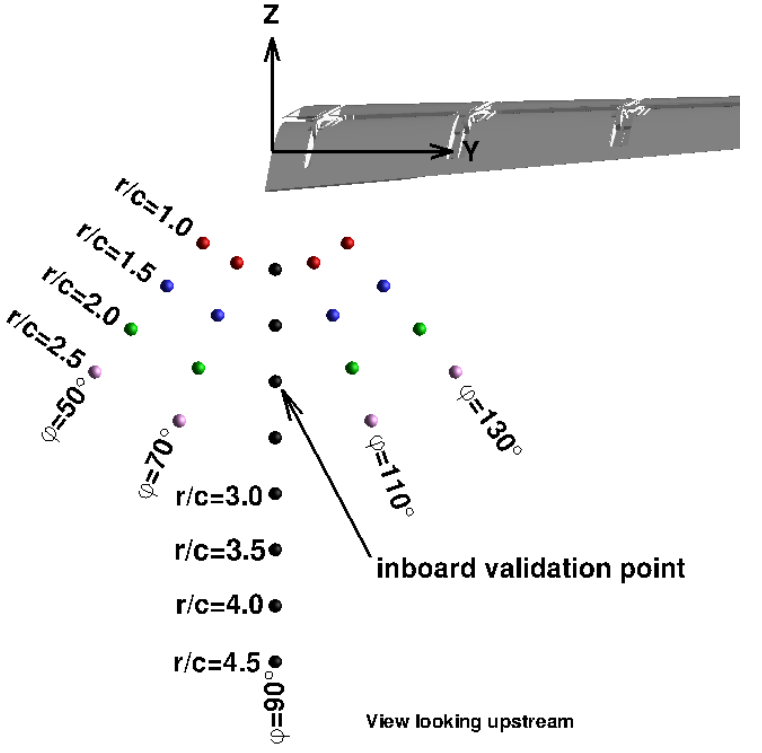

b) Sideline (spanwise) direction

Figure 41. CFD probes arrangement near flap inboard tip for extracting sound field. Probe separation distances are normalized with the inboard tip chord length, $c$.

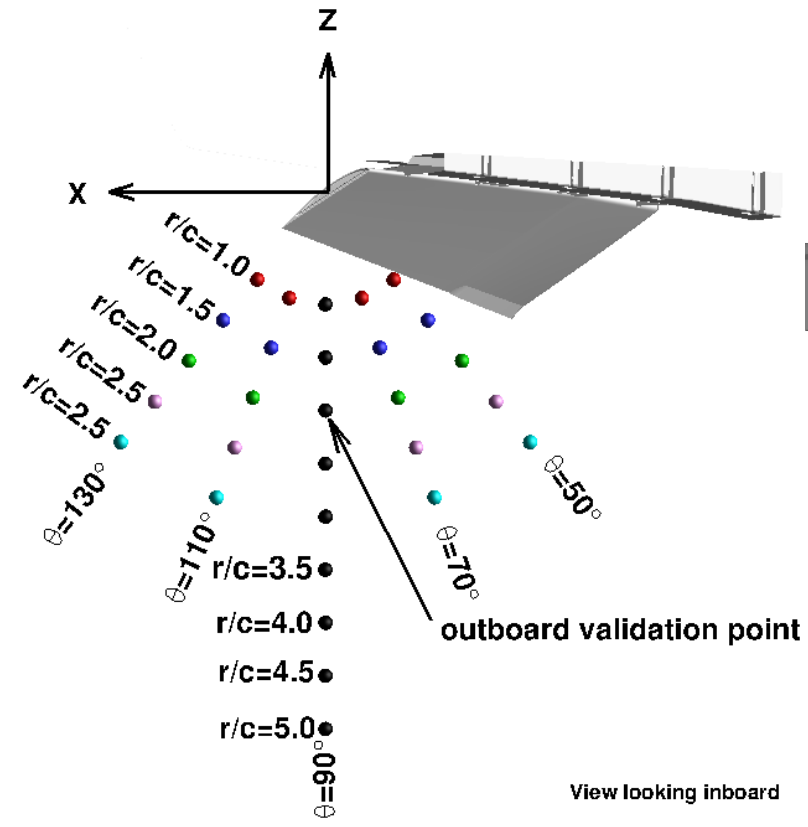

a) Flyover (streamwise) direction

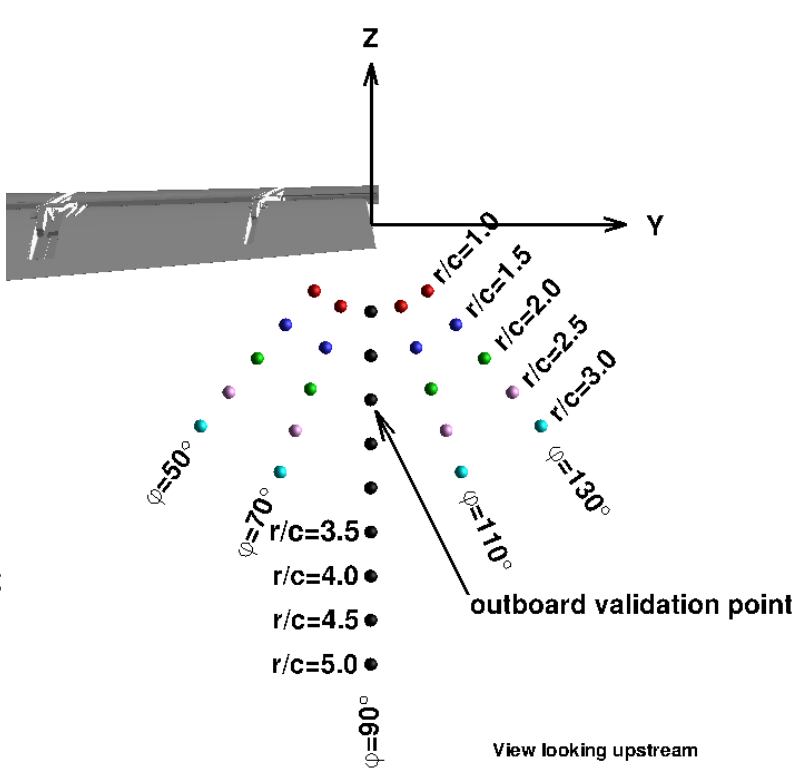

b) Sideline (spanwise) direction

Figure 42. CFD probes arrangement near flap outboard tip for extracting sound field. Probe separation distances are normalized with the outboard tip chord length, $c$. 


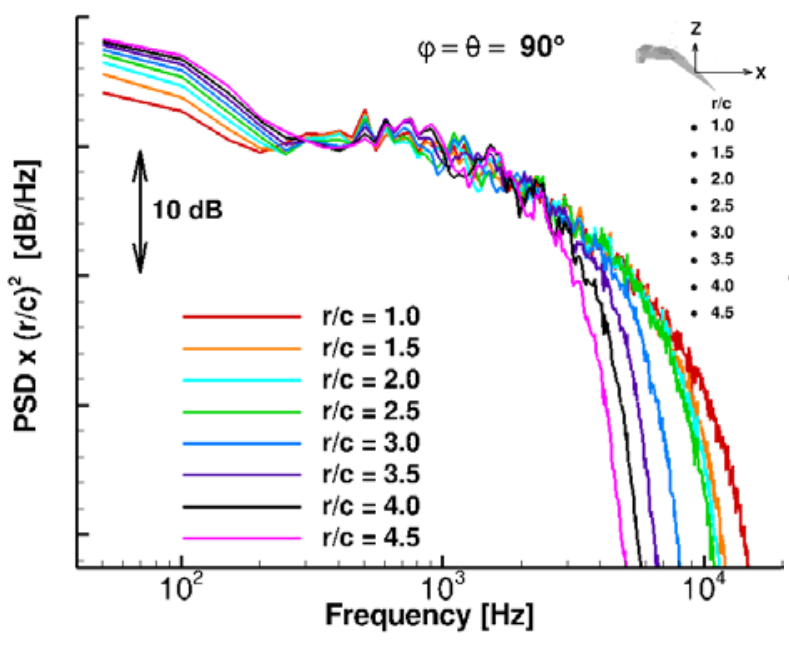

a) Flap inboard tip

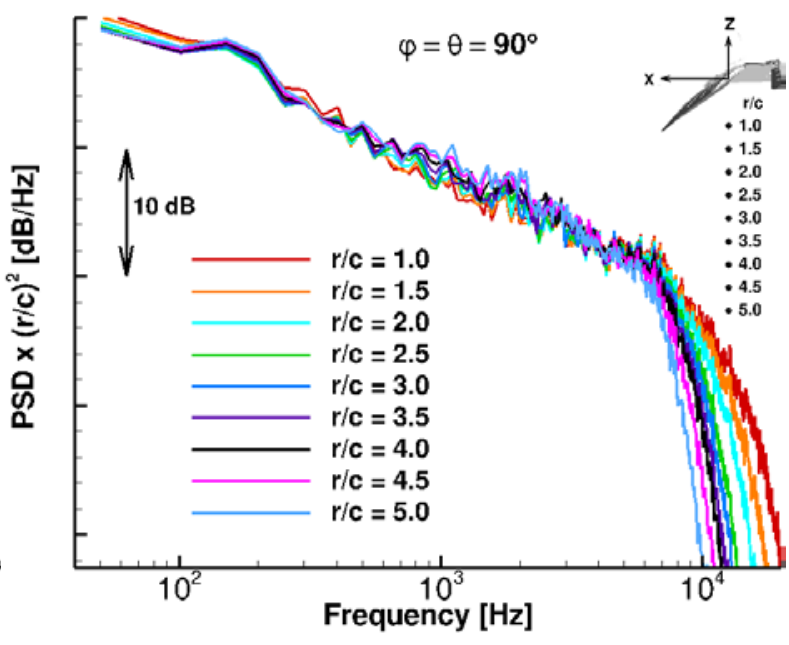

b) Flap outboard tip

Figure 43. Normalized pressure field in the overhead direction demonstrating spherical spreading of waves

\section{Farfield Noise}

The farfield acoustic behavior was obtained using the FW-H approach described in Refs. 28 and 29. The simulated unsteady pressure fields on the entire surface of the $18 \%$ Gulfstream aircraft model were collected at every time step for the two baseline configurations: 59,300 time steps (0.15s record length) and 80,100 time steps (0.2s record length) for main gear off and on, respectively. To validate the FW-H approach and to assess the appropriateness of neglecting the volumetric (quadrupole) sources in the vicinity of the flap tips, the acoustic fields at select probe locations in the overhead direction were obtained first. The inboard and outboard probes at $r / c=2.0$ along the ray $\theta=90^{\circ}$ were used for this purpose. The selected probe locations are identified in Figs. 41 and 42. The PSD analyses were computed by averaging multiple blocks of 8,000 time steps, with each block overlapping the previous and following ones (when possible) by $50 \%$. Thirteen averaging blocks were used for the gear off configuration; the longer time record for the gear on case allowed 17 blocks to be used in the averaging process.

A comparison of the acoustic fields extracted directly from the FUN3D output and those computed by the FW-H approach using the unsteady pressures on the model solid surfaces (excluding the tunnel floor) are compared in Fig. 44. Excellent agreement between the two sets of results is obtained for frequencies up to 8 to $9 \mathrm{kHz}$. As expected, the CFD results decay faster at higher frequencies due to the spatial resolution (diffusion) effects on the propagating waves. The very good agreement observed in these plots clearly indicates that a) the FW-H integration is executed properly and b) volumetric sources do not contribute substantially to the noise radiated from the flap tips.

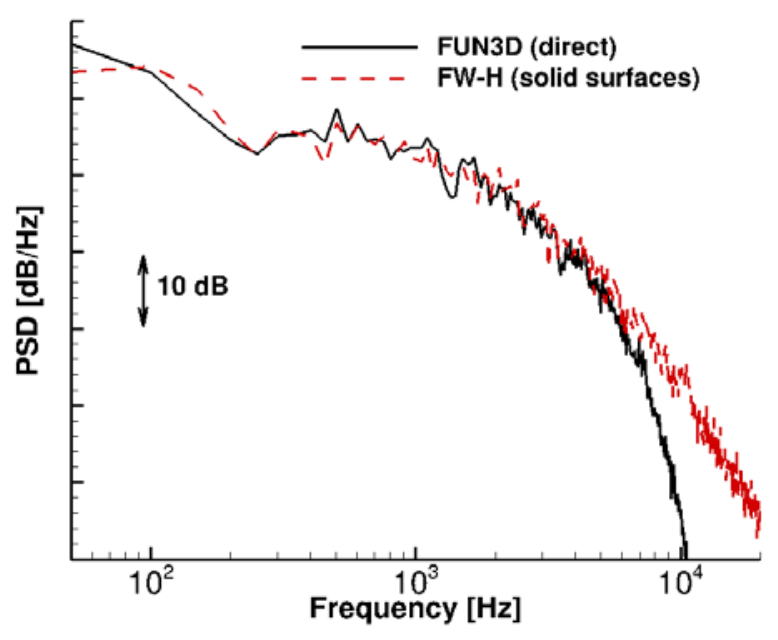

a) Flap inboard tip

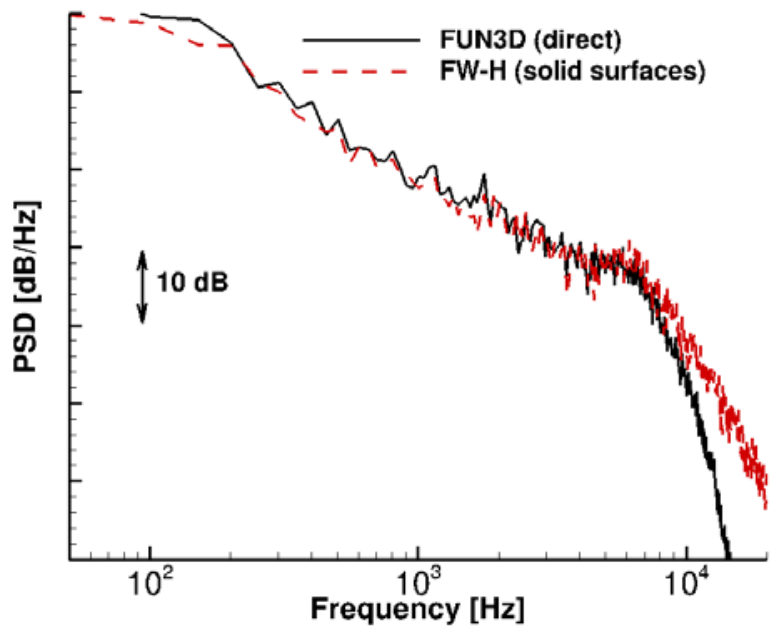

b) Flap outboard tip

Figure 44. Comparison of pressure field at select validation points near the model obtained from FW-H approach using data on solid surfaces and direct extracted from CFD results. 
The farfield noise for the $18 \%$ scale, semi-span Gulfstream aircraft model was obtained recently in the LaRC 14x22 wind tunnel. As detailed in Khorrami et al., ${ }^{11}$ extensive acoustic measurements were acquired using a phased microphone array. The array was positioned on a set of tracks in the flyover direction $17.5 \mathrm{ft}(5.334 \mathrm{~m})$ away from the model, as shown in Fig. 1. During the test, the traversing array allowed measurements within the $50^{\circ}$ to $110^{\circ}$ polar angles. Noise spectra for $\theta=90^{\circ}$ (overhead) at the corresponding array position are plotted in Fig. 45. Two runs from the 14x22 test displaying the repeatability of the measured noise levels are used in this comparison. The measured spectra were obtained from integration of the microphone array beamform maps corresponding to a region of the tunnel test section covering the entire semi-span model. Although the peak of the noise spectrum resides at frequencies below $200 \mathrm{~Hz}$, the portion relevant to environmental noise concerns corresponds to model scale frequencies of $800 \mathrm{~Hz}$ and above. Note that the microphone array was designed for an operational frequency range of approximately $1.5 \mathrm{kHz}$ to $80 \mathrm{kHz}$. Therefore, data measured below $1000 \mathrm{~Hz}$ should be used with caution.

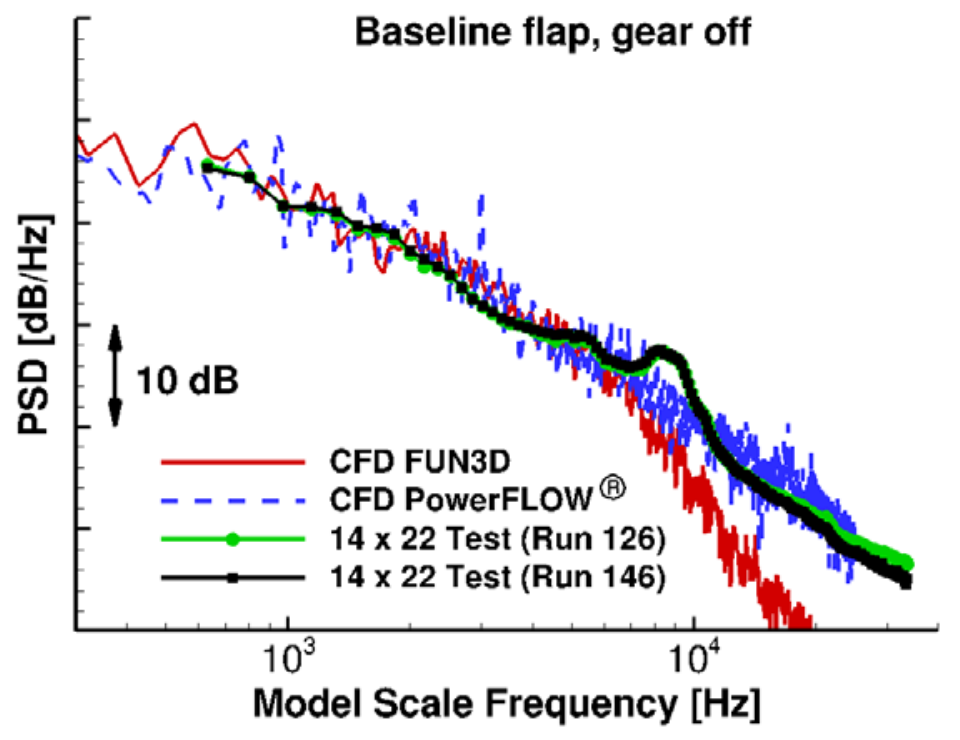

Figure 45. Comparison of far field noise spectrum for $39^{\circ}$ flap deflection and gear off (landing) configuration with microphone array measurements. Computed spectra are based on FW-H approach using pressure data on model solid surfaces.

The computed farfield noise spectrum at the $90^{\circ}$ flyover array position for the baseline case of $39^{\circ}$ flap deflection with the main gear off is compared to the array measurements in Fig. 45. For completeness, the spectrum from the companion Lattice Boltzmann (PowerFLOW®) simulations of Ref. 20 is also shown on the figure. The higher fluctuation amplitude apparent in the computed spectra is related mainly to the shortness of the simulated time records not enough time segments are available to generate smoother averages that are comparable to those of the test results. Inspection of the farfield noise data in Fig. 45 reveals that, for frequencies up to $8 \mathrm{kHz}$, excellent agreement was obtained between both simulations and between each and the measured spectrum - predicted spectrum averages lie within a decibel of the experimental data. At higher frequencies, the FUN3D simulation displays significant decay in the energy levels. We believe that this faster roll-off is less of a spatial resolution issue and more a result of the inadequacy of the MDDES methodology used in this study to capture the unsteady flow field at the flap outboard tip region. In contrast, the PowerFLOW ${ }^{\circledR}$ computed PSD spectrum closely tracks the measured data up to the highest frequency shown $(25 \mathrm{kHz})$. Notice the presence of a broad tonal feature in the measurements occurring between $7 \mathrm{kHz}$ and $11 \mathrm{kHz}$ with a peak near $8 \mathrm{kHz}$. This feature is associated with the cavity at the flap outboard tip. Both simulations fail to capture the tonal aspect of the measured spectrum. As stated earlier, precisely matching all the subtle but relevant geometrical details at the outboard tip of the tested model in the simulated geometry is a very difficult task.

A comparison among predicted and measured noise spectra for the baseline configuration with the main landing gear installed is provided in Fig. 46. As was the case for the flap only configuration, the PSD curves display very good agreement between the two computational approaches and between each simulation and the measured data. With the main gear deployed, both computed spectra slightly over-predict the experimental values by about two 
decibels over most of the frequency range. The FUN3D-based PSD curve shows a faster roll-off at frequencies above $8 \mathrm{kHz}$ due to limitations in the current MDDES methodology and possibly spatial resolution effects. Nevertheless, the results presented in Figs. 45 and 46 provide ample evidence that accurate prediction of the broadband component of airframe noise for a full aircraft is clearly within reach.

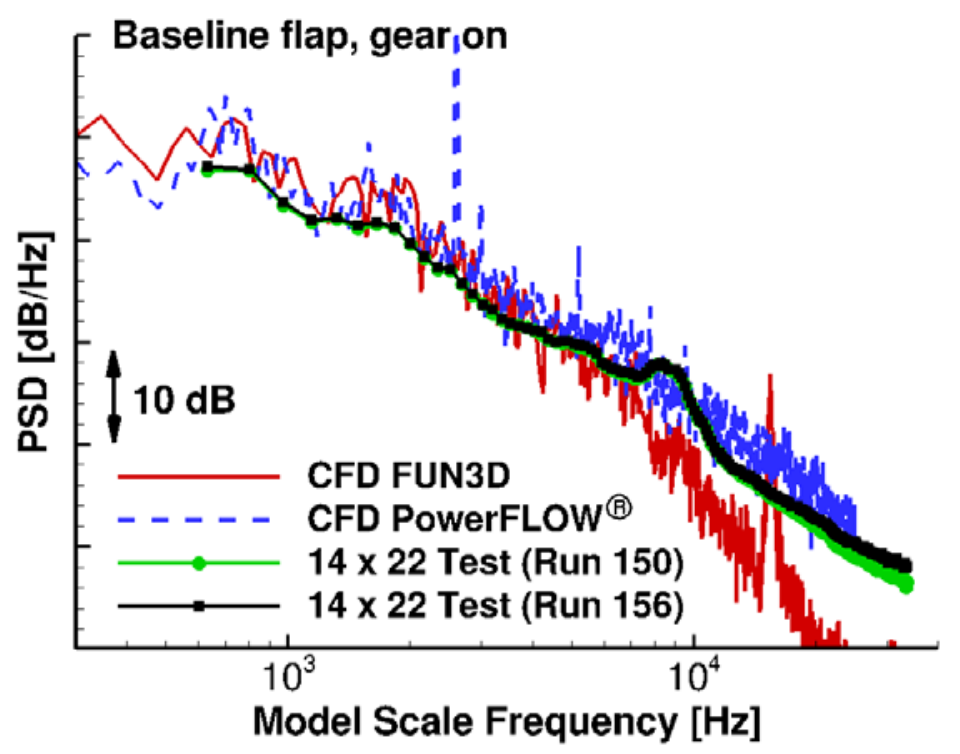

Figure 46. Comparison of farfield noise spectrum for $39^{\circ}$ flap deflection and gear on (landing) configuration with microphone array measurements. Computed spectra are based on FW-H approach using pressure data on model solid surfaces.

The simulations detailed in this paper generally over-predict (in some cases by significant amounts) the measured surface pressure fluctuation levels at both flap tips (see Figs. 29 through 36). In contrast, the Lattice Boltzmann based simulations presented in the companion paper of Khorrami et al. ${ }^{20}$ accurately capture the surface pressure spectra at every available sampling location. Despite these differences, the farfield noise spectra calculated from both sets of computational data are nearly identical over a wide range of frequencies and in close agreement with the measurements. This is a very puzzling issue and should be investigated further. Unless the local spatial correlations for the surface pressure fluctuations obtained from the two solvers are significantly different, it will be difficult to explain the observed agreement in farfield noise. Further scrutiny of the surface pressure correlations at both tips from the FUN3D and PowerFLOW ${ }^{\circledR}$ databases is ongoing. The findings of that study will be reported at a later date.

\section{Concluding Remarks}

This paper documents an extensive computational study aimed at advancing simulation-based airframe noise prediction to include a full aircraft geometry in landing configuration. Towards achieving that goal, the computational effort was centered on conducting high-fidelity time-accurate simulations of an $18 \%$ scale aircraft model that was tested in the NASA LaRC 14x22 wind tunnel. The as-built model used in the present study is a close replica of a Gulfstream aircraft and features many of the finer details of the full scale geometry.

NASA's FUN3D unstructured compressible Navier-Stokes solver was used to perform the computations for free-air at a Mach number of 0.2. The Modified Delayed Detached Eddy Simulation (MDDES) capability of FUN3D (with the Spalart Allmaras turbulence model) was employed during the calculations. Experimental results for two baseline landing configurations consisting of $39^{\circ}$ flap deflection with and without the main landing gear deployed were used to validate the computed solutions. The baseline configuration with main gear off was used during the initial set of simulations that targeted a) the development of a suitable grid, b) identification of proper mesh clustering/distribution in the critical regions, c) selection of appropriate mesh spacing for resolving prominent unsteady flow features, and d) assessment of solution dependency on grid characteristics.

Extensive comparisons with available aeroacoustic measurements were performed. The time-averaged surface pressures on the wing and winglet showed good agreement with the measured data. Very good agreement was also obtained for the steady pressures at the inboard tip and middle portion of the flap. The dual vortex system and the 
resulting surface pressure imprint at the inboard tip were predicted accurately. However, the computed pressure coefficients showed a zone of flow separation in the vicinity of the flap outboard tip that is larger than measurements indicate. This resulted in less than satisfactory agreement for the pressures at the outboard tip. These discrepancies are thought to be most likely caused by limitations in the MDDES hybrid RANS/LES turbulence modeling approach used in this study rather than improper grid resolution.

Predicted surface pressure fluctuation spectra at the flap inboard tip were found to be in reasonable agreement with the measured data regarding frequency content and spectral shape. However, at most probe locations, the magnitude of the pressure fluctuations was over-predicted. A noticeable deterioration in the accuracy of the predicted surface pressure spectra was observed at the flap outboard tip. Increased spatial resolution yielded marginal improvements in the accuracy of the predicted results for the outboard tip.

The configuration with $39^{\circ}$ flap deflection and main landing gear deployed was used to represent a more complete aircraft geometry during landing. Gear-flap interaction effects were shown to be limited to the flap inboard tip region, corroborating measurements of the model in the $14 \times 22$ wind tunnel at NASA LaRC. Changes in the flap steady loading at the inboard tip due to the presence of the gear were predicted accurately. The fluctuating surface pressure spectra were found to be in much better agreement with the measured data than those computed for the configuration with gear off. As was observed in the experimental data, the computed surface pressure fluctuations on the inboard flap edge side wall and top surface showed elevated levels when the main gear was deployed. The simulated time-averaged circumferential pressure distribution on the inner and outer wheels accurately matched the measured values with the stagnation point and peak suction levels on both wheels properly captured. Comparison of the predicted fluctuating surface pressure spectra on the landing gear struts, wheels, and door with the measured data revealed good agreement for the low to mid frequency range (up to $3 \mathrm{kHz}$ ) with the computations decaying faster at higher frequencies.

The success of any airframe noise simulation depends on how well the broadband farfield noise spectrum is predicted. For the configuration with the main gear off, the predicted farfield spectrum for the $90^{\circ}$ overhead position was in remarkable agreement with the microphone array measurements. For frequencies up to $8 \mathrm{kHz}$, the simulation based acoustic intensity were within $1 \mathrm{~dB}$ of the measured values, falling well within the uncertainty limits of the microphone array. For frequencies above $8 \mathrm{kHz}$, the computed spectrum showed a faster roll-off in the acoustic energy levels; this is being attributed to the observed deficiencies in the simulated steady and unsteady pressure fields at the flap outboard tip. Computed farfield acoustic results for the landing configuration with the main gear on also compared well with the measurements. For this configuration, the predicted values were within $2 \mathrm{~dB}$ of the experimental data.

\section{Acknowledgments}

This work was supported by the Environmentally Responsible Aviation (ERA) project under the Integrated System Research Program (ISRP) of NASA. The authors gratefully acknowledge Michael R. Wiese of Vigyan, Inc. for generating and refining the grids used during the computations. Our thanks go to Scott Brynildsen, also of Vigyan, Inc., for providing geometry modifications and CAD support. We would also like to express our sincere appreciation for the support provided by Patrick Moran of NASA Ames Research Center for the high quality visualizations and animations of the large data sets created by us. All the simulations presented here were performed on the Pleiades supercomputer at the NASA Advanced Supercomputing (NAS) facility at Ames Research Center. The logistical support provided by the NAS staff is greatly appreciated.

\section{References}

${ }^{1}$ Michel, U., Barsikow, B., Helbig, J., Hellmig, M., and Schüttpelz, M., “Flyover Noise Measurements on Landing Aircraft with a Microphone Array,” AIAA Paper 98-2336, May 1998.

${ }^{2}$ Piet, J.-F., Elias, G., and Lebigot, P., “Localization of Acoustic Sources from a Landing Aircraft with a Microphone Array,” AIAA Paper 99-1811, May 1999.

${ }^{3}$ Pott-Pollenske, M., Dobrzynski, W., Buchholz, H., Gehlhar, B., and Walle, F., "Validation of a Semiempirical Airframe Noise Prediction Method through Dedicated A319 Flyover Noise Measurements,” AIAA Paper 2002-2470, June 2002.

${ }^{4}$ Stoker, R., Guo, Y., Streett, C. L., and Burnside, N., “Airframe Noise Source Locations of a 777 Aircraft in Flight and Comparisons with Past Model Tests,” AIAA Paper 2003-3232, May 2003.

${ }^{5}$ Elkoby, R., Brusniak, L., Stoker, R., Khorrami, M. R., Abeysinghe, A., and Moe, J. W., “Airframe Noise Results from the QTD II Flight Test Program,” AIAA Paper 2007-3457, May 2007.

${ }^{6}$ Khorrami, M. R., Lockard, D. P., Humphreys, Jr., W. M., Choudhari, M. M., and Van de Ven, T., "Preliminary Analysis of Acoustic Measurements from the NASA-Gulfstream Airframe Noise Flight Test,” AIAA Paper 2008-2814, May 2008. 
${ }^{7}$ Horne, W. C., James, K. D., Arledge, T. K., Soderman, P. T., Burnside, N., and Jaeger, S. M., “Measurements of 26\%-Scale 777 Airframe Noise in the NASA Ames 40- by 80-Foot Wind Tunnel,” AIAA Paper 2005-2810, May 2005.

${ }^{8}$ Jaeger, S. M., Burnside, N. J., Soderman, P. T., Horne, W. C., and James, K. D., “Microphone Array Assessment of an Isolated, 26\%-Scale, High Fidelity Landing Gear,” AIAA Paper 2002-2410, June 2002.

${ }^{9}$ Khorrami, M. R., Hannon, J. A., Neuhart, D. H., Markowski, G. A., and Van de Ven, T., “Aeroacoustic Studies of a HighFidelity Aircraft Model: Part 1-Steady Aerodynamic Measurements,” AIAA Paper 2012-2233, June 2012.

${ }^{10}$ Khorrami, M. R. and Neuhart, D. H, "Aeroacoustic Studies of a High-Fidelity Aircraft Model: Part 2- Unsteady Surface Pressures,” AIAA Paper 2012-2234, June 2012.

${ }^{11}$ Khorrami, M. R., Humphreys, W. M., Lockard, D. P., and Ravetta, P. A., “Aeroacoustic Evaluation of Flap and Landing Gear Noise Reduction Concepts,” Paper to be presented at the $20^{\text {th }}$ AIAA/CEAS Aeroacoustics Conference in Atlanta, GA, June 2014.

${ }^{12}$ Neuhart, D., Hannon, J., and Khorrami, M. R., "Aerodynamic Measurements of a Gulfstream Aircraft Model with and without Noise Reduction Concepts," Paper to be presented at the $20^{\text {th }}$ AIAA/CEAS Aeroacoustics Conference in Atlanta, GA, June 2014.

${ }^{13}$ Yao, C.-S., Jenkins, L. N, Bartram, S. M., Harris, J., Khorrami, M. R., and Mace, W. D., "Flow-Field Investigation of GearFlap Interaction on a Gulfstream Aircraft Model,” Paper to be presented the $20^{\text {th }}$ AIAA/CEAS Aeroacoustics Conference in Atlanta, GA, June 2014.

${ }^{14}$ Vatsa, V., Lockard, D. P., and Khorrami, M. R., “Application of FUN3D Solver for Aeroacoustic Simulation of a Nose Landing Gear Configuration,” AIAA Paper 2011-2820, June 2011.

${ }^{15}$ Vatsa, V., Lockard, D. P., Khorrami, M. R., and Carlson, J.-R., “Aeroacoustic Simulation of a Nose Landing Gear in a Open-Jet Facility using FUN3D,” AIAA Paper 2012-2280, June 2012.

${ }^{16}$ Li. Y., Satti, R., Lew, P.-T., Shock, R., and Noelting, S., "Computational Aeroacoustic Analysis of Flow Around a Complex Nose Landing Gear Configuration,” AIAA Paper 2008-2916, May 2008.

${ }^{17}$ Keating, A., Dthioux, P., Satti, R., Noelting, S., Louis, J., Van de Ven, T., and Vieito, R., “Computational Aeroacoustics Validation and Analysis of a Nose Landing Gear,” AIAA Paper 2009-3154, May 2009.

${ }^{18}$ Fares, E. and Noelting, S., "Unsteady Flow Simulation of a High-Lift Configuration using a Lattice-Boltzmann Approach," AIAA Paper 2011-0869, January 2011.

${ }^{19}$ Casalino, D., Noelting, S., Fares, E., Vand de Ven, T., Perot, F., and Bres, G., “Towards Numerical Aircraft Noise Certification: Analysis of a Full-Scale Landing Gear in Fly-Over Configuration,” AIAA Paper 2012-2235, June 2012.

${ }^{20}$ Khorrami, M. R., Fares, E., and Casalino, D., "Towards Full-Aircraft Airframe Noise Prediction: Lattice-Boltzmann Simulations,” Paper to be presented at the 20 $0^{\text {th }}$ AIAA/CEAS Aeroacoustics Conference in Atlanta GA, June 2014.

${ }^{21}$ Spalart, P. R. and Allmaras, S. R., “A One-Equation Turbulence Model for Aerodynamics Flows,” AIAA Paper 92-0439, 1992.

${ }^{22}$ Dacles-Mariani, J., Rogers, S., Kwak, D., Zilliac, G., and Chow, J., “A Computational Study of Wingtip Vortex Flowfield,” AIAA Paper 93-3010, July 1993.

${ }^{23}$ Menter, F., “Improved Two-Equation k- $\omega$ Turbulence Models for Aerodynamic Flows,” NASA TM 103975, 1992.

${ }^{24}$ Pirzadeh, S. Z., “Advanced Unstructured Grid Generation for Complex Aerodynamic Applications,” AIAA J., Vol. 48, No. 5, pp. 904-915, 2010.

${ }^{25}$ Pirzadeh, S. Z. and Frink, N. T., “Assessment of the Unstructured Grid Software TetrUSS for Drag Prediction of the DLRF4 Configuration,” AIAA Paper 2002-0839, January 2002.

${ }^{26}$ Radeztsky, R. H., Singer, B. A., and Khorrami, M. R., “Detailed Measurements of a Flap Side-Edge Flow Field,” AIAA Paper 98-0700, 1998.

${ }^{27}$ Khorrami, M. R., Singer, B. A., and Radeztsky, R. H., "Reynolds-Averaged Navier-Stokes Computations of a Flap-SideEdge Flowfield,” AIAA J., Vol. 37, No. 1, pp. 14-22, 1999.

${ }^{28}$ Brentner, K. S., Lopes, L. V., Chen, H. N., and Horn, J. F., "Near Real-Time Simulation of Rotorcraft Acoustics and Flight Dynamics," 59 ${ }^{\text {th }}$ Annual Forum, AHS International, Alexandria, VA, 2003.

${ }^{29}$ Bres, G. A., Brentner, K. S., Perez, G., and Jones, H. E., “Maneuvering Rotorcraft Noise Prediction,” Journal of Sound and Vibration, Vol. 275, No. 3-5, pp. 719-738, 2004. 